\title{
Impact of heavy quark masses on parton distributions and LHC phenomenology
}

\author{
NNPDF Collaboration \\ Richard D. Ball ${ }^{\text {a }}$, Valerio Bertone ${ }^{\mathrm{c}}$, Francesco Cerutti ${ }^{\mathrm{d}}$, \\ Luigi Del Debbio ${ }^{a}$, Stefano Forte ${ }^{\mathrm{b}, *}$, Alberto Guffanti ${ }^{\mathrm{c}}$, José I. Latorre ${ }^{\mathrm{d}}$, \\ Juan Rojo ${ }^{\mathrm{b}}$, Maria Ubiali ${ }^{\mathrm{e}}$ \\ a School of Physics and Astronomy, University of Edinburgh, JCMB, KB, Mayfield Rd, Edinburgh EH9 3JZ, Scotland, \\ United Kingdom \\ b Dipartimento di Fisica, Università di Milano and INFN, Sezione di Milano, Via Celoria 16, I-20133 Milano, Italy \\ c Physikalisches Institut, Albert-Ludwigs-Universität Freiburg, Hermann-Herder-Straße 3, \\ D-79104 Freiburg i. B., Germany \\ d Departament d'Estructura i Constituents de la Matèria, Universitat de Barcelona, Diagonal 647, \\ E-08028 Barcelona, Spain \\ e Institut für Theoretische Teilchenphysik und Kosmologie, RWTH Aachen University, D-52056 Aachen, Germany
}

Received 17 January 2011; received in revised form 28 February 2011; accepted 24 March 2011

Available online 29 March 2011

\begin{abstract}
We present a determination of the parton distributions of the nucleon from a global set of hard scattering data using the NNPDF methodology including heavy quark mass effects: NNPDF2.1. In comparison to the previous NNPDF2.0 parton determination, the dataset is enlarged to include deep-inelastic charm structure function data. We implement the FONLL-A general-mass scheme in the FastKernel framework and assess its accuracy by comparison to the Les Houches heavy quark benchmarks. We discuss the impact on parton distributions of the treatment of the heavy quark masses, and we provide a determination of the uncertainty in the parton distributions due to uncertainty in the masses. We assess the impact of these uncertainties on LHC observables by providing parton sets with different values of the charm and bottom quark masses. Finally, we construct and discuss parton sets with a fixed number of flavors.
\end{abstract}

(C) 2011 Elsevier B.V. All rights reserved.

Keywords: Parton distributions; Quantum chromodynamics; LHC; Collider physics

\footnotetext{
* Corresponding author.

E-mail address: forte@mi.infn.it (S. Forte).
} 


\section{Introduction}

The inclusion of effects related to heavy quark masses in the determination of parton distributions (PDFs) has received an increasing amount of attention over the last few years, driven by the increase in accuracy and reliability in the determination of the PDFs required for phenomenology at the LHC (see e.g. Ref. [1] and references therein). Only a few years ago, PDFs in common use (such as e.g. CTEQ6.1 [2]) were based on the so-called zero-mass variable-flavor number scheme (ZM-VFN), in which heavy quarks decouple at scales below their mass, $Q^{2}<m_{h}^{2}$, but are otherwise treated as massless partons, which amounts to neglecting all contributions of order $m_{h}^{2} / Q^{2}$. While this approximation only applies to heavy quark distributions in the vicinity of their respective thresholds, the ensuing modification of the initial conditions to perturbative evolution for the heavy quark distributions also affects light quark PDFs (the momentum sum rule means that a change in any quark's momentum fraction must be accompanied by corresponding changes in the momentum fractions carried by all other partons). The high-energy behavior of light quark PDFs may then be affected at the level of several percent, and the ensuing shift in predictions for precise high-energy standard candles such as the $W$ and $Z$ cross-sections may be quite significant [3]. Furthermore, observables which depend on the heavy quark distributions (such as the single-top production cross-section, which probes the $b$ distribution) are substantially affected [4].

The purpose of this paper is threefold: first, to present a determination of parton distributions based on the NNPDF methodology [5-10] with heavy quark mass effects included. Second, to provide tools to study uncertainties related to heavy quark masses and more general heavy quark effects. Third, to assess the impact of these uncertainties on phenomenology.

The first goal will be achieved by repeating a next-to-leading order global PDF determination based on exactly the same methodology used in the construction of the NNPDF2.0 PDF set [10], but now with heavy quark mass effects included up to order $\alpha_{s}$ through the so-called FONLL-A scheme [11]. The FONLL method (first suggested in Ref. [12] and generalized to deep-inelastic scattering in Ref. [11]) is especially convenient in that it allows the inclusion of heavy quark mass effects to any desired order in $\alpha_{s}$ and any desired logarithmic order. The FONLL-A version corresponds to the combination of $O\left(\alpha_{s}\right)$ mass effects with NLO evolution equations and coefficient functions: at this NLO- $O\left(\alpha_{s}\right)$ order, the FONLL method coincides with the so-called S-ACOT (simplified [13] ACOT [14]) method, adopted for instance in the CTEQ6.6 [15] and CT10 [16] NLO PDF determinations (the MSTW08 [17] uses the related, but somewhat different Thorne-Roberts method [18], see Ref. [19] for benchmark comparisons of these various approaches). The dataset used here also coincides with that of NNPDF2.0, but supplemented by charm deep-inelastic $F_{2}^{c}$ structure function data.

The second goal will be achieved by providing sets of parton distributions which correspond to different values of the heavy quark masses: the uncertainty related to the choice of the quark mass can then be determined simply by variation of the mass value, while combined PDF $+m_{h}$ uncertainties can be determined by constructing Monte Carlo sets of replicas in which the mass is varied according to a probability distribution (typically Gaussian) with a suitable width, in analogy to what was done in Refs. [20,21] to determine combined PDF $+\alpha_{s}$ uncertainties. We also provide a determination of the correlation between the heavy quark masses and individual PDFs. Finally, we will provide PDF sets with various fixed number of flavors.

The third goal will be achieved by computing PDF and heavy quark mass uncertainties for various LHC standard candles: $W, Z$, Higgs and top production. We will also present a preliminary estimate of theoretical uncertainties related to higher order heavy quark mass corrections. 
The outline of this paper is the following: in Section 2 we discuss the features of the datasets included in the NNPDF2.1 analysis, with emphasis on the ZEUS and H1 data on the charm structure function. Then in Section 3 we review the FONLL scheme of Ref. [11] for the inclusion of heavy quark mass effects in neutral current structure functions and present its generalization to charged current deep-inelastic scattering. In Section 4 we present the NNPDF2.1 PDF set and compare it with previous NNPDF releases and with the other global PDF sets, while in Section 5 we perform the same comparisons for LHC standard candles, thus elucidating the impact of the inclusion of heavy quark mass effects in the NNPDF framework. In Section 6 we explore the impact of the uncertainty on the values of the heavy quark masses both on PDFs themselves and on LHC processes using NNPDF2.1 sets with varying $m_{h}$. Finally, in Section 7 we present NNPDF2.1 sets with various fixed number of flavors. Technical details on the implementation and benchmarking of FONLL neutral and charged current structure functions in the FastKernel computational framework of Ref. [10] are collected in Appendices A and B.

\section{Experimental data}

In this section we discuss the experimental data used for the NNPDF2.1 analysis. First of all we motivate the kinematic cuts that are applied to our dataset. Then we present the details and kinematic coverage of the NNPDF2.1 dataset, with special emphasis on the new charm structure function data. Finally, we discuss the implementation of positivity constraints. These data have been used to generate Monte Carlo replicas, which have been checked to reproduce the statistical features of the original dataset. The replica generation and its testing has been performed in the same way as in previous NNPDF analyses $[8,10]$ and will not be discussed further here.

\subsection{Kinematic cuts}

The NNPDF2.1 dataset has been subjected to some kinematic cuts: specifically, the cut in $W^{2}$ is the same as in previous NNPDF fits, $W_{\min }^{2}=12.5 \mathrm{GeV}^{2}$, but the cut in $Q^{2}$ is slightly higher. While in NNPDF2.0 the cut in $Q^{2}$ for the DIS data was set to be $Q_{\min }^{2}=2 \mathrm{GeV}^{2}$, in the NNPDF2.1 analysis we use a somewhat more restrictive kinematic cut in $Q^{2}$, namely $Q_{\min }^{2}=3 \mathrm{GeV}^{2}$. There are two main motivations for this modification which we now discuss. First, very close to the heavy quark threshold the predictions for $F_{2}^{c}$ from the GM scheme might suffer from instabilities due to the threshold behavior. One would like to avoid having data crossing the charm mass threshold when varying the heavy quark mass in various fits. This suggests to use a value of $Q_{\min }^{2}$ at least as large as the maximum value of the charm mass than can be considered acceptable. $Q_{\min }^{2}=3 \mathrm{GeV}^{2}$ is then a reasonable choice since then $m_{c}^{\max } \sim 1.7 \mathrm{GeV}$. Furthermore, there is now an indication of possible deviations from NLO DGLAP in the small$x$ and $Q^{2}$ HERA data [22,23]. These deviations are mostly relevant in the smaller $Q^{2}$ bins of HERA data. The theoretical uncertainty in the PDFs and LHC observables related to their inclusion in the global fit is moderate as compared to the PDF errors and other uncertainties, but removing the HERA points below $Q_{\min }^{2}$ reduces these theoretical uncertainties even further. The price to pay for this reduced theoretical uncertainty is an increase in statistical uncertainty: indeed, we will see in Section 4.3 that removing the data below $Q_{\min }^{2}=3 \mathrm{GeV}^{2}$ results in an increase of PDF uncertainty in the small- $x$ gluon PDF due to the reduced experimental information.

On top of the previous general kinematic cuts, applied to all the DIS experiments, we will also perform additional cuts on the HERA $F_{2}^{c}$ data. The motivation for these is that in this work we will use the FONLL-A general-mass scheme for heavy quarks, and as discussed in [11], 
Table 1

The values of the initial evolution scale where the PDFs are parametrized, $Q_{0}^{2}$, and the kinematic cuts in $Q^{2}$ and $W^{2}$ applied to the fitted DIS dataset, $Q_{\min }^{2}$ and $W_{\min }^{2}$, in the present work and in other recent PDF determinations. As discussed in the text, further cuts are applied to $F_{2}^{c}$ data in the NNPDF2.1 case. For HERAPDF the value of $W_{\text {min }}^{2}$ given is the minimum of the HERA dataset and no cut is performed.

\begin{tabular}{lllc}
\hline & $Q_{0}^{2}\left[\mathrm{GeV}^{2}\right]$ & $Q_{\min }^{2}\left[\mathrm{GeV}^{2}\right]$ & $W_{\min }^{2}\left[\mathrm{GeV}^{2}\right]$ \\
\hline NNPDF2.1 & 2.0 & 3.0 & 12.5 \\
NNPDF2.0 [10] & 2.0 & 2.0 & 12.5 \\
CT10 [16] & 1.69 & 4.0 & 12.25 \\
MSTW08 [17] & 1 & 2.0 & 15.0 \\
ABKM09 [24] & 9 & 2.5 & 3.24 \\
HERAPDF1.0 [25] & 1.9 & 3.5 & 155.75 \\
\hline
\end{tabular}

FONLL-A ${ }^{1}$ provides a poor description of the data in the smallest $x$ and $Q^{2}$ bins due to missing large $\mathcal{O}\left(\alpha_{s}^{2}\right)$ corrections. Only the FONLL-B scheme can cure this problem since it includes consistently $\mathcal{O}\left(\alpha_{s}^{2}\right)$ corrections in $F_{2}^{c}$ into an NLO fit, as can be seen in [11] and we will review in Section 3. We will thus remove from the fit HERA $F_{2}^{c}$ data with $Q^{2} \leqslant 4 \mathrm{GeV}^{2}$ and data with $Q^{2} \leqslant 10 \mathrm{GeV}^{2}$ for $x \leqslant 10^{-3}$. These cuts ensure that all $F_{2}^{c}$ experimental data included in the fit are well described by $\mathcal{O}\left(\alpha_{s}\right)$ theory.

In Table 1 we summarize the choices for the initial evolution scale and kinematic cuts applied in this work, compared to the choices in other recent PDF determinations. Note that HERAPDF does not perform a cut in $W^{2}$ since they only include HERA data which do not extend to the low- $W^{2}$ region.

\subsection{NNPDF2.1 dataset}

Now we discuss the datasets that are included in the present analysis. As compared to the NNPDF2.0 analysis [10], on top of all relevant data from DIS, Drell-Yan and weak vector boson production $^{2}$ and inclusive jet production we include here all the relevant charm structure function $F_{2}^{c}\left(x, Q^{2}\right)$ data from the H1 and ZEUS experiments at HERA [27-33]. These datasets provide a handle on the small- $x$ gluon, and are sensitive also to the value of the charm mass $m_{c}$. On the other hand, HERA $F_{2}^{b}$ has much larger uncertainties, and is thus not included in the present analysis. The kinematic coverage of all the datasets included in NNPDF2.1 is summarized in Table 2 and in Fig. 1. Note that the only differences with respect to the NNPDF2.0 dataset are the addition of HERA $F_{2}^{c}$ data and the new kinematic cut $Q_{\min }^{2}=3 \mathrm{GeV}^{2}$.

Now we describe in turn the features of the various $F_{2}^{c}\left(x, Q^{2}\right)$ datasets included in the present analysis. For most experimental sets the full correlation is not available and thus one is forced to add in quadrature systematic and statistical uncertainties. The full correlation matrix for all data points, including the cross-correlations between datasets and between H1 and ZEUS will be provided together with the combined HERA $F_{2}^{c}$ dataset: this combination will thus significantly improve the accuracy of the existing separate datasets.

\footnotetext{
1 Note that this is true for any heavy quark scheme that does not include the $\mathcal{O}\left(\alpha_{s}^{2}\right)$ corrections, like for example the S-ACOT- $\chi$ used in the CTEQ/CT family of PDF sets.

2 The impact of the leptonic W asymmetry data from the Tevatron, not included in NNPDF2.0, has been studied in Ref. [26] using the Bayesian reweighting technique.
} 
Table 2

Experimental datasets included in the NNPDF2.1 global analysis. For DIS experiments we provide in each case the number of data points and the ranges of the kinematic variables before and after (in parenthesis) kinematic cuts. For hadronic data we show the ranges of parton $x$ covered for each set determined using leading order parton kinematics. Note that hadronic data are unaffected by kinematic cuts. The values of $x_{\min }$ and $Q_{\min }^{2}$ for the total dataset hold after imposing kinematic cuts.

\begin{tabular}{|c|c|c|c|c|c|c|c|}
\hline Experiment & Set & Ref. & $N_{\text {dat }}$ & $x_{\min }$ & $x_{\max }$ & $Q_{\min }^{2}\left[\mathrm{GeV}^{2}\right]$ & $Q_{\max }^{2}\left[\mathrm{GeV}^{2}\right]$ \\
\hline \multicolumn{8}{|c|}{ Deep-inelastic scattering } \\
\hline \multirow[t]{2}{*}{ NMC-pd } & & & $260(132)$ & & & & \\
\hline & NMC-pd & [34] & $260(132)$ & $0.0015(0.008)$ & 0.68 & $0.2(3.5)$ & 99.0 \\
\hline \multirow[t]{2}{*}{ NMC } & & & $288(221)$ & & & & \\
\hline & NMC & [35] & $288(221)$ & $0.0035(0.009)$ & 0.47 & $0.8(3.2)$ & 61.2 \\
\hline \multirow[t]{3}{*}{ SLAC } & & & $422(74)$ & & & & \\
\hline & SLACp & [36] & $211(37)$ & $0.07(0.1)$ & $0.85(0.55)$ & $0.58(3.0)$ & 29.2 \\
\hline & SLACd & [36] & $211(37)$ & $0.07(0.1)$ & $0.85(0.55)$ & $0.58(3.2)$ & 29.1 \\
\hline \multirow[t]{3}{*}{ BCDMS } & & & $605(581)$ & & & & \\
\hline & BCDMSp & [37] & $351(333)$ & 0.07 & 0.75 & 7.5 & 230.0 \\
\hline & BCDMSd & [38] & $254(248)$ & 0.07 & 0.75 & 8.8 & 230.0 \\
\hline \multirow[t]{5}{*}{ HERAI-AV } & & & $741(592)$ & & & & \\
\hline & HERA1-NCep & [25] & $528(379)$ & $6.2 \times 10^{-7}\left(4.3 \times 10^{-5}\right)$ & 0.65 & $0.045(3.5)$ & 30000 \\
\hline & HERA1-NCem & [25] & 145 & $1.3 \times 10^{-3}$ & 0.65 & 90.000 & 30000 \\
\hline & HERA1-CCep & [25] & 34 & 0.008 & 0.4 & 300.0 & 15000 \\
\hline & HERA1-CCem & [25] & 34 & 0.013 & 0.4 & 300.0 & 30000 \\
\hline \multirow[t]{3}{*}{ CHORUS } & & & $1214(862)$ & & & & \\
\hline & CHORUSnu & [39] & $607(431)$ & $0.02(0.045)$ & 0.65 & $0.3(3.0)$ & 95.2 \\
\hline & CHORUSnb & [39] & $607(431)$ & $0.02(0.045)$ & 0.65 & $0.3(3.0)$ & 95.2 \\
\hline \multirow[t]{2}{*}{ FLH108 } & & & 8 & & & & \\
\hline & FLH108 & [40] & 8 & 0.00028 & 0.0036 & 12.0 & 90.000 \\
\hline \multirow[t]{3}{*}{ NTVDMN } & & & $90(79)$ & & & & \\
\hline & NTVnuDMN & {$[41,42]$} & $45(41)$ & 0.027 & 0.36 & $1.1(3.1)$ & 116.5 \\
\hline & NTVnbDMN & {$[41,42]$} & $45(38)$ & 0.021 & 0.25 & $0.8(3.1)$ & 68.3 \\
\hline \multirow[t]{3}{*}{ ZEUS-H2 } & & & 127 & & & & \\
\hline & $\mathrm{Z} 06 \mathrm{NC}$ & [43] & 90 & $5 \times 10^{-3}$ & 0.65 & 200 & $3 \times 10^{5}$ \\
\hline & $\mathrm{Z} 06 \mathrm{CC}$ & [44] & 37 & 0.015 & 0.65 & 280 & $3 \times 10^{5}$ \\
\hline
\end{tabular}


Table 2 (Continued.)

\begin{tabular}{|c|c|c|c|c|c|c|c|}
\hline Experiment & Set & Ref. & $N_{\text {dat }}$ & $x_{\min }$ & $x_{\max }$ & $Q_{\min }^{2}\left[\mathrm{GeV}^{2}\right]$ & $Q_{\max }^{2}\left[\mathrm{GeV}^{2}\right]$ \\
\hline \multicolumn{8}{|c|}{ HERA charm structure function data } \\
\hline \multirow[t]{5}{*}{ ZEUSF2C } & & & $69(50)$ & & & & \\
\hline & ZEUSF2C99 & {$[27]$} & $21(14)$ & $5 \times 10^{-5}\left(3 \times 10^{-4}\right)$ & 0.02 & $1.8(7.0)$ & 130 \\
\hline & ZEUSF2C03 & [28] & $31(21)$ & $3 \times 10^{-5}\left(1.8 \times 10^{-5}\right)$ & 0.03 & $2.0(7.0)$ & 500 \\
\hline & ZEUSF2C08 & [29] & $9(7)$ & $2.2 \times 10^{-4}\left(6.5 \times 10^{-4}\right)$ & 0.032 & 7.0 & 112 \\
\hline & ZEUSF2C09 & {$[30]$} & 8 & $8 \times 10^{-4}$ & 0.03 & 30 & 1000 \\
\hline \multirow[t]{4}{*}{$\mathrm{H} 1 \mathrm{~F} 2 \mathrm{C}$} & & & $47(38)$ & & & & \\
\hline & $\mathrm{H} 1 \mathrm{~F} 2 \mathrm{C} 01$ & {$[31]$} & $12(6)$ & $5 \times 10^{-4}$ & $3.2 \times 10^{-3}$ & $1.5(12)$ & 60 \\
\hline & $\mathrm{H} 1 \mathrm{~F} 2 \mathrm{C} 09$ & {$[32]$} & 6 & $2.4 \times 10^{-4}$ & 0.025 & 120 & 400 \\
\hline & $\mathrm{H} 1 \mathrm{~F} 2 \mathrm{C} 10$ & {$[33]$} & 26 & $2 \times 10^{-4}\left(3.2 \times 10^{-4}\right)$ & 0.05 & $5.0(12)$ & 2000 \\
\hline Experiment & Set & Ref. & $N_{\text {dat }}$ & {$\left[y / x_{\min }^{F}, y / x_{\max }^{F}\right]$} & {$\left[x_{\min }, x_{\max }\right]$} & $M_{\min }^{2}\left[\mathrm{GeV}^{2}\right]$ & $M_{\max }^{2}\left[\mathrm{GeV}^{2}\right]$ \\
\hline \multicolumn{8}{|c|}{ Fixed target Drell-Yan production } \\
\hline \multirow[t]{2}{*}{ DYE605 } & & & 119 & & & & \\
\hline & DYE605 & {$[45]$} & 119 & {$[-0.20,0.40]$} & {$[0.14,0.65]$} & 50.5 & 286 \\
\hline \multirow[t]{3}{*}{ DYE866 } & & & 390 & & & & \\
\hline & DYE866p & {$[46,47]$} & 184 & {$[0.0,0.78]$} & {$[0.017,0.87]$} & 19.8 & 251.2 \\
\hline & DYE866r & {$[48]$} & 15 & {$[0.05,0.53]$} & {$[0.025,0.56]$} & 21.2 & 166.4 \\
\hline Experiment & Set & Ref. & $N_{\text {dat }}$ & {$\left[y_{\min }, y_{\max }\right]$} & {$\left[x_{\min }, x_{\max }\right]$} & $M_{\min }^{2}\left[\mathrm{GeV}^{2}\right]$ & $M_{\max }^{2}\left[\mathrm{GeV}^{2}\right]$ \\
\hline \multicolumn{8}{|c|}{ Collider vector boson production } \\
\hline \multirow[t]{2}{*}{ CDFWASY } & & & 13 & & & & \\
\hline & CDFWASY & [49] & 13 & {$[0.10,2.63]$} & {$\left[2.9 \times 10^{-3}, 0.56\right]$} & 6463 & 6463 \\
\hline \multirow[t]{2}{*}{ CDFZRAP } & & & 29 & & & & \\
\hline & CDFZRAP & {$[50]$} & 29 & {$[0.05,2.85]$} & {$\left[2.9 \times 10^{-3}, 0.80\right]$} & 8315 & 8315 \\
\hline \multirow[t]{2}{*}{ D0ZRAP } & & & 28 & & & & \\
\hline & DOZRAP & {$[51]$} & 28 & {$[0.05,2.75]$} & {$\left[2.9 \times 10^{-3}, 0.72\right]$} & 8315 & $\begin{array}{l}8315 \\
\text { ued on next page }\end{array}$ \\
\hline
\end{tabular}


Table 2 (Continued.)

\begin{tabular}{|c|c|c|c|c|c|c|c|}
\hline Experiment & Set & Ref. & $N_{\text {dat }}$ & {$\left[y_{\min }, y_{\max }\right]$} & {$\left[x_{\min }, x_{\max }\right]$} & $p_{T, \min }^{2}\left[\mathrm{GeV}^{2}\right]$ & $p_{T, \max }^{2}\left[\mathrm{GeV}^{2}\right]$ \\
\hline \multicolumn{8}{|c|}{ Collider inclusive jet production } \\
\hline \multirow[t]{2}{*}{ CDFR2KT } & & & 76 & & & & \\
\hline & CDFR2KT & {$[52]$} & 76 & {$[0.05,1.85]$} & {$\left[4.6 \times 10^{-3}, 0.90\right]$} & 3364 & $3.7 \times 10^{5}$ \\
\hline \multirow[t]{2}{*}{ D0R2CON } & & & 110 & & & & \\
\hline & D0R2CON & [53] & 110 & {$[0.20,2.20]$} & {$\left[3.1 \times 10^{-3}, 0.97\right]$} & 3000 & $3.4 \times 10^{5}$ \\
\hline \multicolumn{3}{|l|}{ Experiment } & $N_{\text {dat }}$ & $x_{\min }$ & $x_{\max }$ & $Q_{\min }^{2}\left[\mathrm{GeV}^{2}\right]$ & $Q_{\max }^{2}\left[\mathrm{GeV}^{2}\right]$ \\
\hline \multicolumn{3}{|l|}{ TOTAL } & $4520(3415)$ & $3.1 \times 10^{-5}$ & 0.97 & 2.0 & $3.7 \times 10^{5}$ \\
\hline
\end{tabular}




\section{NNPDF2.1 dataset}

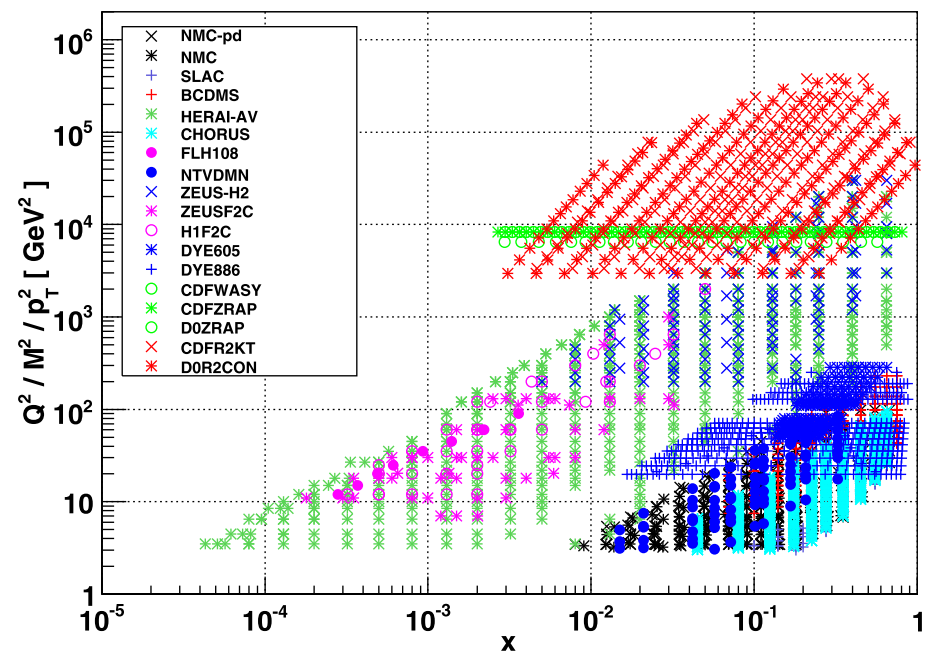

Fig. 1. Experimental datasets which enter the NNPDF2.1 analysis. The kinematic coverage of each dataset is summarized in Table 2 .

The $F_{2}^{c}$ data which we use in the NNPDF2.1 analysis are the following:

- The ZEUS 96-97 $D^{* \pm}$ analysis [27].

In this analysis $F_{2}^{c}$ is extracted from the measurement of $D^{* \pm}$ mesons reconstructed via their hadronic decays using data collected in the 1996 and 1997 running periods.

- The ZEUS 98-00 $D^{*}$ analysis [28].

As in the previous case, $F_{2}^{c}$ is extracted from the measurement of $D^{* \pm}$ mesons reconstructed via their hadronic decays, and uses data collected in the running period between 1998 and 2000.

- The 04-05 ZEUS $D^{ \pm}, D^{0}$ analysis [29].

In this analysis, based on the HERA-II running period of 2004 and 2005, D mesons are reconstructed via their hadronic decays. An improved precision is obtained reducing the combinatorial background to the $D$ meson signals by using the ZEUS micro-vertex detector to reconstruct displaced secondary vertices.

- The 2005 ZEUS muon analysis [30].

This dataset is based on the measurement of muons that are generated in charm production from their semileptonic decays. Data was collected during the 2005 HERA-II running period.

- The H1 96-97 $D^{* \pm}$ analysis [31].

This analysis, based on the 1996-1997 running period, used similar reconstruction strategies as the corresponding ZEUS analysis, namely the reconstruction of $D^{* \pm} \rightarrow D^{0} \pi^{+}$using the $D^{*}-D^{0}$ mass difference method.

- The H1 large $Q^{2} 04-07 D^{* \pm}$ analysis [32].

This analysis determines $F_{2}^{c}$ via identified $D$ mesons produced at large virtualities $Q^{2} \geqslant$ $100 \mathrm{GeV}^{2}$, and is based on data collected in the HERA-II running period 2004-2007.

- The H1 low- $Q^{2} 06-07 D^{* \pm}$ analysis [33]. 
This is analogous to the previous measurement, but now covering the small and medium $Q^{2}$ region. It is based on data obtained in the HERA-II 2006-2007 running period. Events containing heavy quarks are distinguished from those containing only light quarks using variables that are sensitive to the longer lifetimes of heavy flavor hadrons, like the transverse displacement of tracks from the primary vertex.

There are more published $F_{2}^{c}$ datasets from HERA but the ones that are included here supersede previous obsolete measurements and are the basis of the combined HERA $F_{2}^{c}$ dataset. In Section 4 we will quantify the impact of the HERA $F_{2}^{c}$ data onto the PDFs.

A concern with $F_{2}^{c}$ data which has been sometimes used to motivate their exclusion from PDF determinations is the fact that the way $F_{2}^{c}$ is usually defined experimentally, as the contribution to $F_{2}$ with at least one charmed quark in the final state, is affected by mass singularities (i.e., it is not finite in the limit in which $m_{c} \rightarrow 0$ ). Here we will adopt a definition of $F_{2}^{c}$ (as the contribution to $F_{2}^{c}$ when only the charm electric charge is nonzero) which is free of mass singularities; the deviation between this definition and that which is used to define the experimental observable is estimated in Ref. [11] by means of a suitable resummation method, and shown to be negligible in the region of the HERA data. Also, $F_{2}^{c}$ is affected by theoretical uncertainties related to the extrapolation from the experimentally accessible region (restricted in $p_{T}$ and $\eta$ ) to the full phase space. This theoretical uncertainty is estimated using QCD exclusive partonic calculations and added as an extra source of systematic uncertainty in the experimental analysis.

\subsection{Positivity constraints}

As discussed in [10], within the NNPDF framework general theoretical constraints can be imposed guaranteeing that the fitting procedure only explores the subspace of acceptable physical solutions. An important theoretical constraint is the positivity of physical cross-sections. As discussed in Ref. [54], positivity should be imposed on observable hadronic cross-sections and not on partonic quantities, which do not necessarily satisfy this constraint (except at leading order where the probabilistic interpretation holds). Positivity constraints may be implemented in various ways; here we will impose them through Lagrange multipliers, i.e. in practice by adding pseudo-datasets for physical cross-sections with extremely small uncertainties in such a way that negative cross-sections would lead to a very large contribution to the $\chi^{2}$.

In NNPDF2.1 we impose positivity of the following observables:

- The longitudinal structure function $F_{L}\left(x, Q^{2}\right)$, which constrains the gluon positivity at small- $x$.

- The charm production cross-section in neutrino DIS, $d^{2} \sigma^{\nu, c} / d x d y$ [9], which constrains the strange PDFs both at large and at small- $x$, beyond the reach of existing data.

- The neutral current DIS charm structure function $F_{2}^{c}\left(x, Q^{2}\right)$, useful to impose the positivity of the gluon at very large- $x$, where it is not constrained by any experimental dataset.

All the positivity constraints are implemented at a low scale $Q_{\mathrm{pos}}^{2}$ that we take to be $Q_{\mathrm{pos}}^{2}=$ $2 \mathrm{GeV}^{2}$, in the range $x \in\left[10^{-6}, x_{\max }\right]$, where $x_{\max }$ is the corresponding kinematic boundary, $x_{\max } \sim 0.1$ for NC scattering and $x_{\max } \sim 0.5$ for CC scattering. DGLAP evolution then takes care of preserving the positivity properties for higher scales. We note that the physical observables for the pseudo-data that implement the positivity constraints are computed consistently at the same 
perturbative order as all other physical observables, in the present case next-to-leading order perturbative QCD.

\section{Structure functions with heavy quark mass effects}

The FONLL-A general-mass scheme was introduced for neutral current structure functions in Ref. [11]. We begin this section with a brief review of this scheme, emphasizing the impact of heavy quark effects on DIS structure functions. We then discuss the values of the heavy quark masses and the associated uncertainties adopted in the present analysis. The corresponding analysis for charged current structure functions is presented in the last part of this section.

\subsection{The FONLL-A general-mass scheme for NC structure functions}

The FONLL general-mass scheme, originally proposed in the context of heavy quark photoand hadro-production, was generalized in Ref. [11] to deep-inelastic structure functions. We refer the reader to Ref. [11] for a detailed discussion of the scheme, and for the notation adopted in this section. The FONLL approach allows for a consistent combination of terms determined in a massive, or decoupling, or fixed-flavor number (FFN) scheme, in which the heavy quark is subtracted at zero momentum (rather than in the $\overline{\mathrm{MS}}$ scheme), so it decouples for scales much below its mass, and it is included in Feynman diagrams up to some fixed order in $\alpha_{s}$ above the threshold for its production, with terms determined in a massless, or zero-mass (ZM), or simply $\overline{\mathrm{MS}}$ scheme, in which the heavy flavor is treated as another massless parton, so it is included in the all-order resummation of collinear logarithms, up to a suitable chosen logarithmic order (LO, NLO, etc.). A significant feature of the FONLL method is that the fixed perturbative order of the FFN computation and the resummed logarithmic order of the ZM computation which are being combined can be chosen independently of each other.

In the present analysis, we combine FFN massive terms up to order $\alpha_{s}$ with an NLO ZM computation; this is called FONLL-A in Ref. [11]. As shown in Ref. [19], this turns out to be identical to the S-ACOT [13] scheme used in recent CTEQ/CT PDF determinations [15,16]. Once a specific "general-mass" (GM) scheme for the combination of FFN and ZM terms has been chosen, there is still a freedom in the treatment of subleading terms: indeed, it turns out to be phenomenologically convenient to suppress subleading terms near the quark threshold (see Ref. [11,55]). In this work we adopt the so-called threshold or damping factor method of Ref. [11] for the treatment of subleading terms. In Ref. [19] the damping factor method is benchmarked against various implementations of the alternative, commonly used $\chi$-scaling method for the treatment of subleading terms.

We now present the explicit expressions for the $F_{2, h}$ heavy quark structure function. ${ }^{3}$ The FONLL-A heavy quark structure function is given by the sum of two terms:

$$
F_{2, h}^{\mathrm{FONLL}}\left(x, Q^{2}\right)=F_{2, h}^{\left(n_{l}\right)}\left(x, Q^{2}\right)+\theta\left(Q^{2}-m_{h}^{2}\right)\left(1-\frac{m_{h}^{2}}{Q^{2}}\right)^{2} F_{2, h}^{(d)}\left(x, Q^{2}\right) .
$$

The first contribution on the right-hand side of Eq. (1) is the massive-scheme heavy quark structure function at $\mathcal{O}\left(\alpha_{s}\right)$ :

\footnotetext{
3 See Ref. [11] for the discussion on the FONLL expressions for the longitudinal structure functions.
} 


$$
F_{2, h}^{\left(n_{l}\right)}\left(x, Q^{2}\right)=x \int_{x}^{1} \frac{d y}{y} C_{2, g}^{\left(n_{l}\right)}\left(\frac{x}{y}, \frac{Q^{2}}{m_{h}^{2}}, \alpha_{s}\left(Q^{2}\right)\right) g^{\left(n_{l}+1\right)}\left(y, Q^{2}\right) .
$$

The heavy quark gluon coefficient function is given by

$$
C_{2, g}^{\left(n_{l}\right)}\left(z, \frac{Q^{2}}{m_{h}^{2}}, \alpha_{s}\left(Q^{2}\right)\right)=\frac{\alpha_{s}\left(Q^{2}\right)}{2 \pi} 2 e_{h}^{2} C_{2, g}^{\left(n_{l}\right), 1}\left(z, \frac{Q^{2}}{m_{h}^{2}}\right)+\mathcal{O}\left(\alpha_{s}^{2}\right) .
$$

The $\mathcal{O}\left(\alpha_{s}\right)$ coefficient is

$$
\begin{aligned}
C_{2, g}^{\left(n_{l}\right), 1}\left(z, \frac{Q^{2}}{m_{h}^{2}}\right)= & \theta\left(W^{2}-4 m_{h}^{2}\right) \times T_{R}\left[\left(z^{2}+(1-z)^{2}+4 \epsilon z(1-3 z)-8 \epsilon^{2} z^{2}\right) \log \frac{1+v}{1-v}\right. \\
& +(8 z(1-z)-1-4 \epsilon z(1-z)) v]
\end{aligned}
$$

where we have defined

$$
\epsilon \equiv m_{h}^{2} / Q^{2}, \quad v \equiv \sqrt{1-4 m_{h}^{2} / W^{2}},
$$

and the partonic center of mass energy $W^{2}=Q^{2}(1-z) / z$.

The second term on the right-hand side of Eq. (1) is the "difference" contribution

$$
\begin{aligned}
F_{2, h}^{(d)}\left(x, Q^{2}\right)= & x \int_{x}^{1} \frac{d y}{y}\left[C_{2, q}^{\left(n_{l}+1\right)}\left(\frac{x}{y}, \alpha_{s}\left(Q^{2}\right)\right)\left[h^{\left(n_{l}+1\right)}\left(y, Q^{2}\right)+\bar{h}^{\left(n_{l}+1\right)}\left(y, Q^{2}\right)\right]\right. \\
& \left.+\left(C_{2, g}^{\left(n_{l}+1\right)}\left(\frac{x}{y}, \alpha_{s}\left(Q^{2}\right)\right)-B_{g, h}^{(0)}\left(\frac{x}{y}, \frac{Q^{2}}{m_{h}^{2}}, \alpha_{s}\left(Q^{2}\right)\right)\right) g^{\left(n_{l}+1\right)}\left(y, Q^{2}\right)\right],
\end{aligned}
$$

where $h, \bar{h}$ are the heavy quark parton distributions; at first order in $\alpha_{s}, B_{g, h}^{(0)}$ is given by

$$
B_{g, h}^{(0), 1}\left(z, \frac{Q^{2}}{m_{h}^{2}}\right)=2 e_{h}^{2} C_{2, g}^{\left(n_{l}, 0\right), 1}\left(z, \frac{Q^{2}}{m_{h}^{2}}\right),
$$

and the massless limit of the massive coefficient function is

$$
C_{2, g}^{\left(n_{l}, 0\right), 1}\left(z, \frac{Q^{2}}{m_{h}^{2}}\right)=T_{R}\left[\left(z^{2}+(1-z)^{2}\right) \log \frac{Q^{2}(1-z)}{m_{h}^{2} z}+(8 z(1-z)-1)\right],
$$

which in the limit $Q^{2}=m_{h}^{2}$ reproduces as required the usual massless scheme coefficient function.

Note that in all terms in Eq. (1) PDFs and $\alpha_{s}$ are expressed in the same factorization scheme, namely, the zero-mass $n_{f}=4$ scheme. Exploiting this fact, it is easy to check explicitly that the "difference" term (6) is formally of higher order near the heavy quark threshold [11] (and thus in particular it can be suppressed using a suitable threshold prescription).

Eq. (1) interpolates smoothly between the massive scheme at small- $Q^{2}$ and the massless scheme suitable at large- $Q^{2}$. As an illustration of the differences between various schemes for the heavy quark structure functions, in Fig. 2 we compare the $F_{2, c}$ and the $F_{L, c}$ charm structure functions for various schemes: ZM, FONLL-A and the FFN scheme as a function of $Q^{2}$ for different values of $x$. It is clear that FONLL-A interpolates smoothly between the FFN scheme 

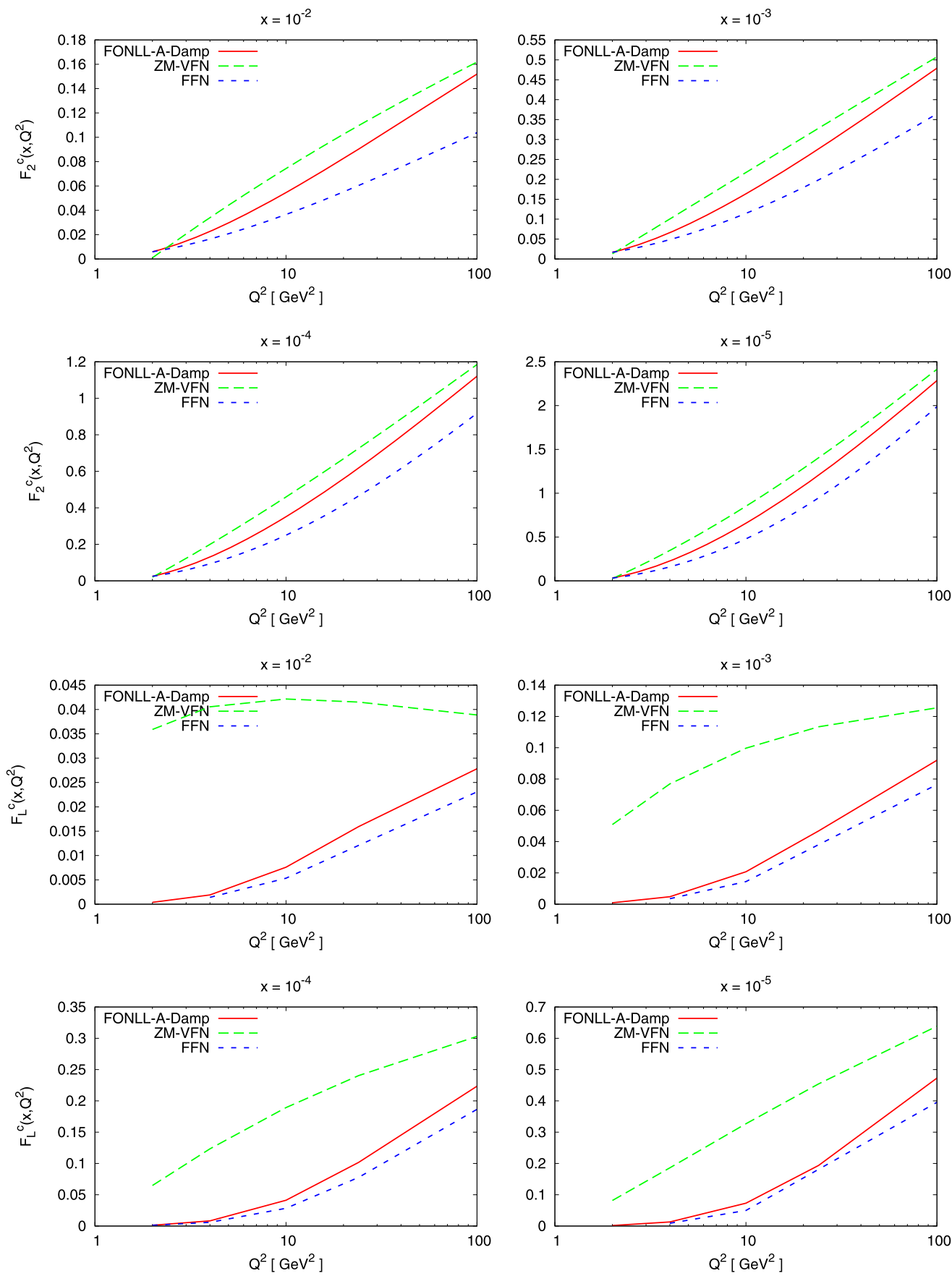

Fig. 2. The charm structure functions $F_{2, c}\left(x, Q^{2}\right)$ and $F_{L, c}\left(x, Q^{2}\right)$ as a function of $Q^{2}$ for different values of $x$ from $x=$ $10^{-5}$ to $x=10^{-2}$ in various heavy quark schemes, computed using the FastKernel method: FONLL-A, ZM-VFN and the FFN schemes. The PDFs and settings are identical to those of the Les Houches heavy quark benchmark comparison. 

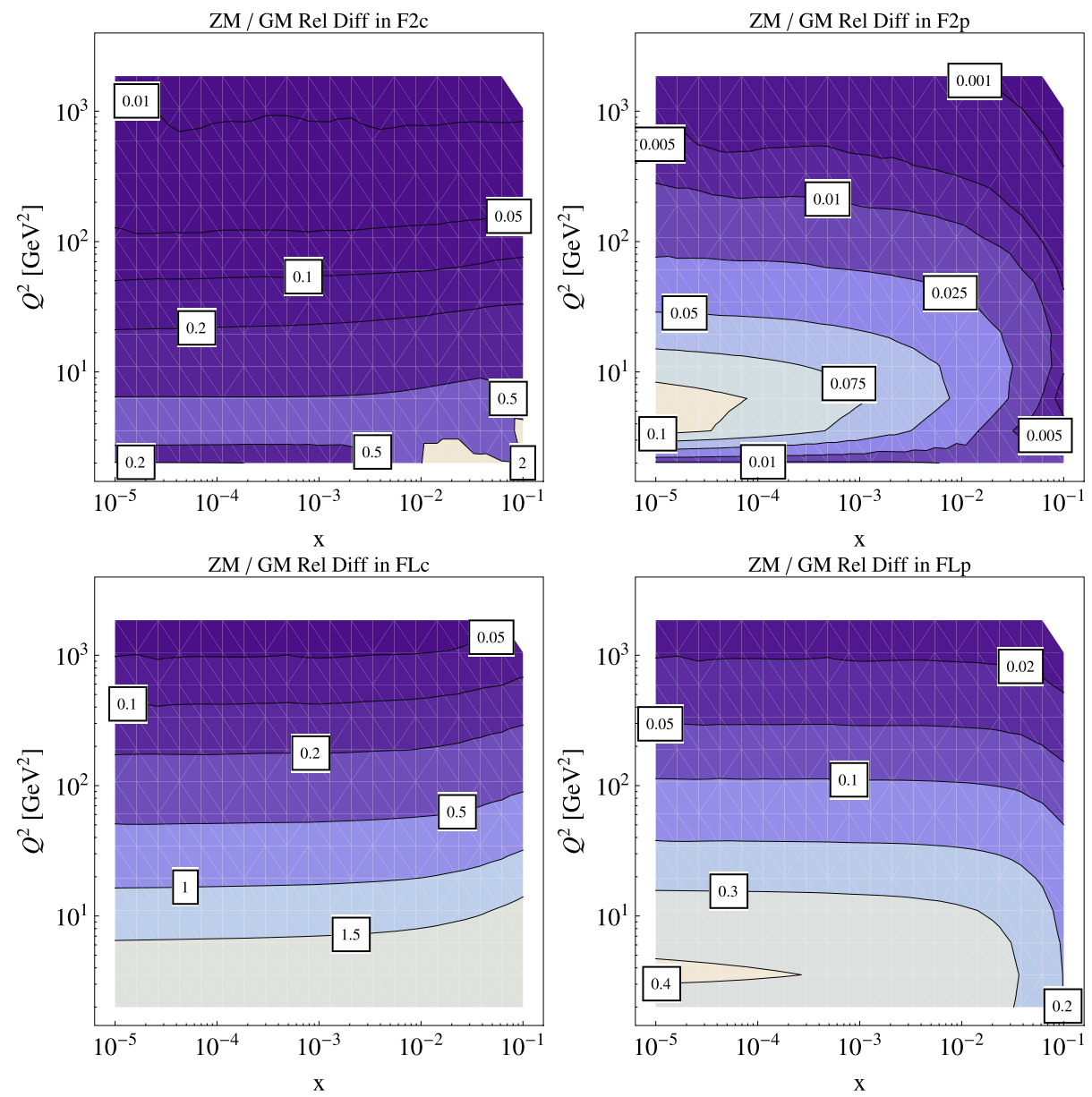

Fig. 3. Left plots: the relative difference for the charm structure functions $F_{2, c}\left(x, Q^{2}\right)$ and $F_{L, c}\left(x, Q^{2}\right)$ computed in the ZM and FONLL-A schemes as a function of $x$ and $Q^{2}$. The PDFs and settings are identical to those of the Les Houches heavy quark benchmark comparison. Right plots: the same but now for the inclusive structure functions $F_{2}^{p}\left(x, Q^{2}\right)$ and $F_{L}^{p}\left(x, Q^{2}\right)$.

near threshold and the massless scheme at large- $Q^{2}$ (also thanks to the use of a damping factor in Eq. (1)). For this comparison, PDFs and other settings, like the value of $m_{c}$, are identical to those of the Les Houches heavy quark benchmark comparison [19]. The comparison for the longitudinal structure function $F_{L, c}$ shows that mass effects are much larger than in $F_{2, c}$, so the ZM computation is completely unreliable.

The impact of heavy quark mass effects in DIS structure functions is further quantified in Fig. 3, where the relative difference between the ZM and FONLL-A schemes is computed as a function of $x$ and $Q^{2}$, both for the inclusive structure functions $F_{2}^{p}$ and $F_{L}^{p}$ and for the charm structure functions $F_{2, c}$ and $F_{L, c}$. For the phenomenologically more relevant case of $F_{2}^{p}$, we see that heavy quark mass effects can be as large as $\sim 10 \%$, decreasing fast for increasing $x$ and $Q^{2}$. As in the case of Fig. 2 the Les Houches heavy quark benchmark settings have been used. Note that while the qualitative features of Fig. 3 are general, the quantitative detail can depend 


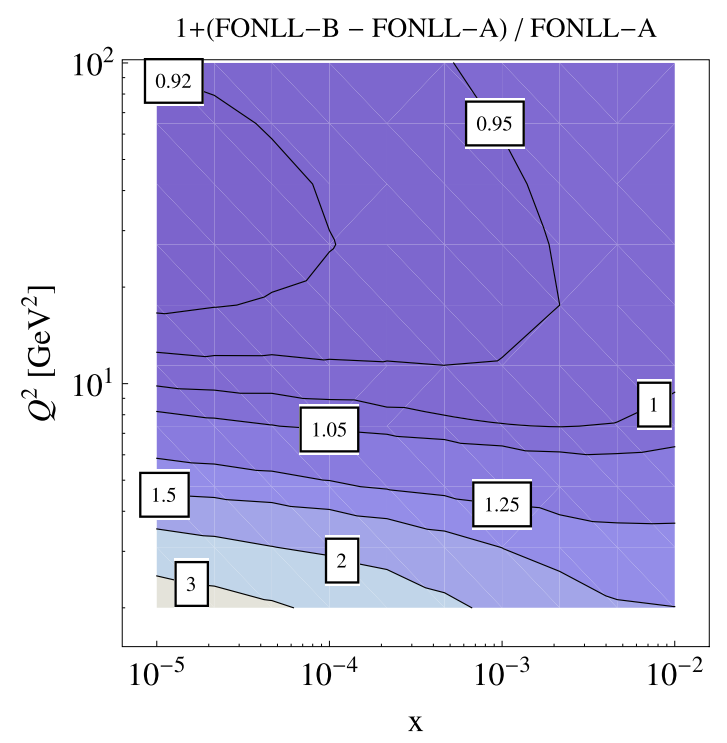

Fig. 4. Relative difference for the charm structure functions $F_{2, c}\left(x, Q^{2}\right)$ between the FONLL-B and FONLL-A generalmass schemes, in units of FONLL-A, as a function of $x$ and $Q^{2}$. The PDFs and settings are identical to those of the Les Houches heavy quark benchmark comparison.

on specific features of the general-mass heavy quark scheme, like for example the prescription to suppress the subleading threshold terms.

As discussed in Ref. [11] it is possible to account for the phenomenologically relevant $\mathcal{O}\left(\alpha_{s}^{2}\right)$ corrections to $F_{2, c}$ into an NLO PDF fit by means of the FONLL-B scheme. We show in Fig. 4 the relative difference between $F_{2, c}$ computed in the FONLL-B and FONLL-A schemes. As shown in the figure, their difference at small- $x$ and $Q^{2}$ is rather large. The inadequacy of $\mathcal{O}\left(\alpha_{s}\right)$ theory to describe the low- $x$ and $Q^{2} F_{2, c}$ data motivates the cuts to the $F_{2, c}$ HERA datasets discussed in Section 2.1. At larger values of $x$ and $Q^{2}$ the differences between the two schemes become of the order of a few percent, much smaller than the typical experimental uncertainties, thus validating the inclusion of the $F_{2, c}$ data into the present fit based on the FONLL-A scheme.

The $\mathcal{O}\left(\alpha_{s}\right)$ massive-scheme heavy quark coefficient function, Eq. (4), was first computed in Refs. [56-58], while its Mellin transform, hitherto not available ${ }^{4}$ is presented in Appendix A. Details of the implementation of the FONLL-A scheme in the FastKernel framework used in the NNPDF analysis are also given in Appendix A. We have assessed the accuracy of the FONLL implementation computing the Les Houches heavy quark benchmark tables [19], showing that the accuracy is sufficient for precision PDF determination.

\subsection{FONLL charged current structure functions}

The FONLL method for charged currents was only mentioned briefly in Ref. [11]: here we provide a detailed explanation of this general-mass scheme for CC structure functions. In the charged current sector, coefficient functions are only known up to $\mathcal{O}\left(\alpha_{s}\right)$, so the FONLL-A

4 A numerical parametrization of the Mellin space heavy quark coefficient functions up to $\mathcal{O}\left(\alpha_{s}^{2}\right)$ was provided in Ref. [59]. 
scheme can be constructed, while the FONLL-B and C which are based on unavailable $\mathcal{O}\left(\alpha_{s}^{2}\right)$ massive results cannot.

Heavy quark mass effects are required to describe charm production in neutrino DIS (the dimuon process) and to a lesser extent also the inclusive neutrino reduced cross-sections, since in both cases most of the data lie close to the charm threshold, $Q^{2} \gtrsim m_{c}^{2}$. HERA charged current data on the other hand are at large- $Q^{2}$ and thus for practical purposes any general-mass scheme reduces to the $\mathrm{ZM}-\mathrm{VFN}$ scheme.

Here we generalize the FONLL-A scheme to charged current structure functions. Its implementation in the FastKernel framework requires the analytic computation of the Mellin transforms of the $\mathcal{O}\left(\alpha_{s}\right)$ charged current heavy quark coefficient functions [62]. The detailed description of the implementation is given in Appendix B. We then benchmark the FONLL implementation in FastKernel, using the Les Houches heavy quark benchmark settings [19] comparing with an $x$-space code written for this purpose that implements FONLL-A for CC structure functions. For simplicity, we will make the assumption that $\left|V_{\mathrm{cs}}\right|=1$, and the rest of the CKM matrix elements are zero. The generalization to realistic CKM elements, as actually implemented in FastKernel, is straightforward. We assume also a single heavy quark, the charm quark with mass $m_{c}$. The factorization scale is set to be equal to $\mu_{F}^{2}=Q^{2}$. Finally, we consider only neutrino induced charm production, the anti-neutrino case is again straightforward.

In the FFN massive scheme, the charged current charm production $F_{2, c}^{\mathrm{CC}}$ structure function for neutrino induced scattering has been computed in $x$ space in Refs. [62,63]:

$$
\begin{aligned}
F_{2, c}^{\left(n_{l}\right), \mathrm{CC}}\left(x, Q^{2}\right)= & 2 \xi s\left(\xi, Q^{2}\right)+2 \xi \frac{\alpha_{s}\left(Q^{2}\right)}{2 \pi}\left\{\int _ { \xi } ^ { 1 } \frac { d z } { z } \left[C_{2, h}^{\left(n_{l}\right), 1}\left(z, Q^{2}, \lambda\right) s\left(\frac{\xi}{z}, Q^{2}\right)\right.\right. \\
& \left.\left.+C_{2, g}^{\left(n_{l}\right), 1}\left(z, Q^{2}, \lambda\right) g\left(\frac{\xi}{z}, Q^{2}\right)\right]\right\},
\end{aligned}
$$

where

$$
\xi=x\left(1+\frac{m_{c}^{2}}{Q^{2}}\right), \quad \lambda \equiv \frac{Q^{2}}{Q^{2}+m_{c}^{2}} .
$$

In Eq. (9), $C_{2, g}^{\left(n_{l}\right), 1}$ includes the contributions in which the gluon splits into an $s$ and a $\bar{c}$ quark, both of which contribute to $F_{2, c}^{\left(n_{l}\right), C C}$ at NLO. The Feynman diagrams for the LO and NLO gluoninduced subprocesses are shown in Figs. 5, 6.

The $x$-space expressions for the $\mathcal{O}\left(\alpha_{s}\right)$ charged current coefficient functions in Eq. (9) are given in Refs. [62,63]. The quark coefficient function can be separated into a delta function piece, a regular piece and a singular piece regulated with the usual plus prescription,

$$
C_{2, h}^{\left(n_{l}\right), 1}\left(z, Q^{2}, \lambda\right)=C_{h, \delta}^{\left(n_{l}\right)}(\lambda) \delta(1-z)+C_{h, \mathrm{r}}^{\left(n_{l}\right)}(\lambda, z)+\left[C_{h, \mathrm{~s}}^{\left(n_{l}\right)}\left(\lambda, z, Q^{2}\right)\right]_{+} .
$$

The explicit expressions for the different pieces are the following. For the delta term we have

$$
\begin{aligned}
& C_{h, \delta}^{\left(n_{l}\right)}(\lambda)=-C_{F}\left(4+\frac{1}{2 \lambda}+\frac{\pi^{2}}{3}+\frac{1+\lambda}{2 \lambda} K_{A}\right), \\
& K_{A}=(1-\lambda) \ln (1-\lambda) / \lambda .
\end{aligned}
$$

\footnotetext{
5 Partial knowledge of $\mathcal{O}\left(\alpha_{S}^{2}\right)$ massive terms is available in the form of the asymptotic [60] and threshold limits [61].
} 


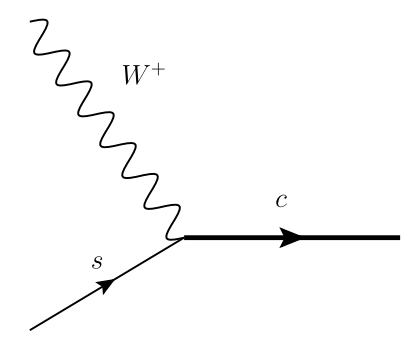

Fig. 5. Feynman diagram for the LO contribution to $F_{2, c}^{\left(n_{l}\right), \mathrm{CC}}$ in the FFN scheme. Thick solid lines indicate a heavy quark (charm) and thin solid lines a light quark (strange).
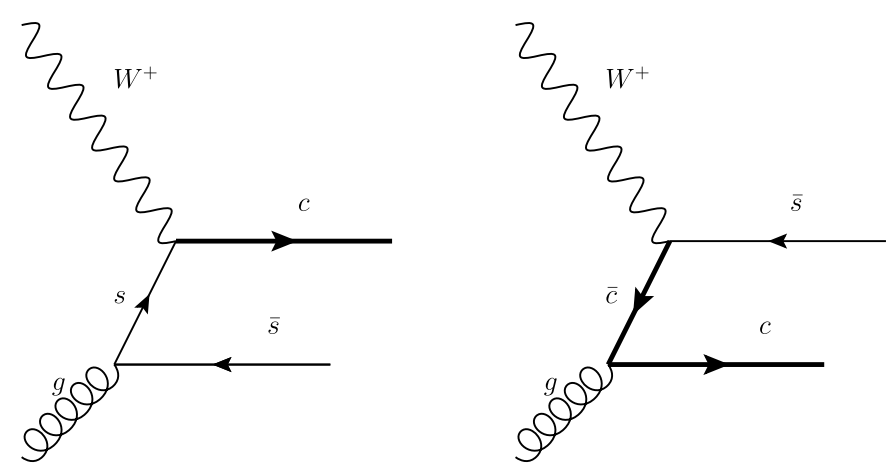

Fig. 6. Feynman diagrams for the $\operatorname{NLO}\left(\mathcal{O}\left(\alpha_{s}\right)\right)$ gluon-induced contribution to $F_{2, c}^{\left(n_{l}\right), \mathrm{CC}}$ in the FFN scheme.

The regular piece can be written as

$$
\begin{aligned}
C_{h, \mathrm{r}}^{\left(n_{l}\right)}(\lambda, z)= & C_{F}\left[-(1+z)(2 \ln (1-z)-\ln (1-\lambda z))-\frac{\left(1+z^{2}\right) \ln z}{1-z}\right. \\
& \left.+\left(2 z+2-\frac{2}{z}\right)+\left(\frac{2}{z}-1-z\right) \frac{1}{1-\lambda z}\right],
\end{aligned}
$$

and finally the singular piece reads

$$
\begin{aligned}
C_{h, \mathrm{~s}}^{\left(n_{l}\right)}\left(\lambda, z, Q^{2}\right)= & C_{F}\left[-\frac{1+z^{2}}{1-z} \ln \frac{Q^{2}+m_{c}^{2}}{Q^{2}}+2 \frac{2 \ln (1-z)-\ln (1-\lambda z)}{1-z}\right. \\
& \left.-\frac{2}{1-z}+\frac{1}{2} \frac{1-z}{(1-\lambda z)^{2}}\right]
\end{aligned}
$$

where the first term is the contribution that depends on the factorization scale and is proportional to the $q q$ splitting function. Separating the massive quark coefficient functions into the various contributions is important to properly evaluate their Mellin transforms, as will be discussed below.

Finally, we give the expression for the FFN gluon coefficient function. In this case there are no singular terms and it reads

$$
\begin{aligned}
C_{2, g}^{\left(n_{l}\right), 1}\left(z, Q^{2}, \lambda\right)= & {\left[T_{f}\left(z^{2}+(1-z)^{2}\right)\left(\ln \frac{1-\lambda z}{(1-\lambda) z}+\ln \frac{Q^{2}+m_{c}^{2}}{Q^{2}}\right)\right.} \\
& +T_{f}\left(z^{2}+(1-z)^{2}\right)(2 \ln (1-z)-\ln (1-\lambda z)-\ln z)
\end{aligned}
$$




$$
\begin{aligned}
& +\left(8-18(1-\lambda)+12(1+\lambda)^{2}\right) z(1-z)+\left(\frac{1-\lambda}{1-\lambda z}-1\right) \\
& \left.+(1-\lambda) z \ln \frac{1-\lambda z}{(1-\lambda) z}\left(6 \lambda-12 \lambda^{2} z\right)\right] .
\end{aligned}
$$

Again the last term in the first line is the scale-dependent contribution and is proportional to $P_{q g}^{(0)}$. Note that both the diagrams shown in Fig. 6 contribute [62]. Analogous expressions for the charged current $F_{3, c}$ and $F_{L, c}$ structure functions can be found in Refs. [62,63].

As in the case of neutral currents, the massless limits of the FFN structure functions are easily obtained. For the massive $F_{2, c}^{\mathrm{CC}}$ structure function it has the structure

$$
\begin{aligned}
F_{2, c}^{\left(n_{l}, 0\right), \mathrm{CC}}\left(x, Q^{2}\right)= & 2 x s\left(x, Q^{2}\right) \\
& +2 x \frac{\alpha_{s}\left(Q^{2}\right)}{2 \pi}\left\{\int _ { x } ^ { 1 } \frac { d z } { z } \left[C_{2, h}^{\left(n_{l}, 0\right), 1}\left(z, Q^{2}, \lambda\right) s\left(\frac{x}{z}, Q^{2}\right)\right.\right. \\
& \left.\left.+C_{2, g}^{\left(n_{l}, 0\right), 1}\left(z, Q^{2}, \lambda\right) g\left(\frac{x}{z}, Q^{2}\right)\right]\right\},
\end{aligned}
$$

where

$$
\begin{aligned}
& C_{2, h}^{\left(n_{l}, 0\right), 1}\left(z, Q^{2}, \lambda\right)=C_{h, \delta}^{\left(n_{l}, 0\right)} \delta(1-z)+C_{h, \mathrm{r}}^{\left(n_{l}, 0\right)}(z)+\left[C_{h, \mathrm{~s}}^{\left(n_{l}, 0\right)}(z)\right]_{+}, \\
& C_{h, \delta}^{\left(n_{l}, 0\right)}=-C_{F}\left(\frac{9}{2}+\frac{\pi^{2}}{3}\right), \\
& C_{h, \mathrm{r}}^{\left(n_{l}, 0\right)}(z)=C_{F}\left[-(1+z) \ln (1-z)-\frac{\left(1+z^{2}\right) \ln z}{1-z}+3+2 z\right], \\
& C_{h, \mathrm{~s}}^{\left(n_{l}, 0\right)}(z)=C_{F}\left[2\left(\frac{\ln (1-z)}{1-z}\right)-\frac{3}{2}\left(\frac{1}{1-z}\right)\right] ;
\end{aligned}
$$

and for the gluon

$$
\begin{aligned}
C_{2, g}^{\left(n_{l}, 0\right), 1}\left(z, Q^{2}\right)= & 2 T_{f}\left[\left(z^{2}+(1-z)^{2}\right) \ln \frac{1-z}{z}+8 z(1-z)-1\right] \\
& +T_{f}\left(z^{2}+(1-z)^{2}\right) \ln \frac{Q^{2}}{m_{c}^{2}} .
\end{aligned}
$$

For completeness, we provide also the ZM-VFN quark coefficient functions for quarks and gluons,

$$
\begin{aligned}
C_{2, h}^{\left(n_{l}+1\right), 1}(z)= & C_{F}\left[2\left(\frac{\ln (1-z)}{1-z}\right)_{+}-\frac{3}{2}\left(\frac{1}{1-z}\right)_{+}\right. \\
& -(1+z) \ln (1-z)-\frac{\left(1+z^{2}\right) \ln z}{1-z}+3+2 z \\
& \left.+\delta(1-z)\left(-\frac{\pi^{2}}{3}-\frac{9}{2}\right)\right], \\
C_{2, g}^{\left(n_{l}+1\right), 1}(z)= & T_{F}\left[\left(z^{2}+(1-z)^{2}\right) \ln \frac{1-z}{z}+(8 z(1-z)-1)\right] .
\end{aligned}
$$



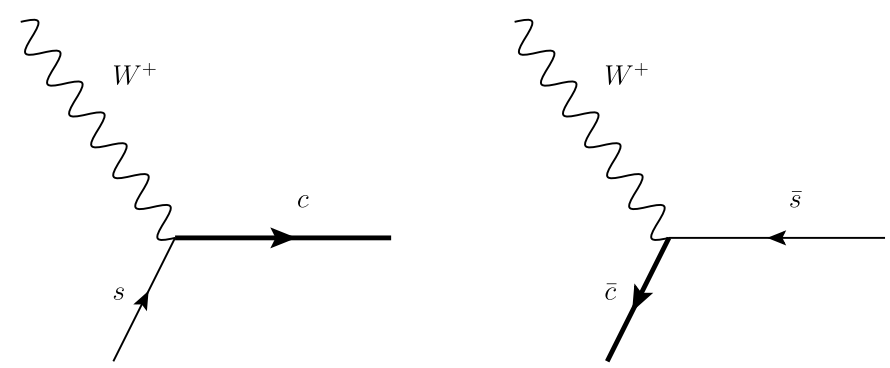

Fig. 7. Feynman diagrams that contribute to $F_{2, c}^{\left(n_{l}+1\right), C C}$ in the ZM-VFN scheme at leading order. The NLO diagrams are the same as in the FFN scheme.

Note that the above gluon coefficient function, Eq. (24), is defined according to the notation of Ref. [64], that is, it corresponds to the production of a single quark or antiquark.

Comparing the FFNS0 and ZM-VFN coefficient functions we find that for the gluon piece the following relation holds

$$
C_{2, g}^{\left(n_{l}, 0\right), 1}\left(z, Q^{2}\right)=2 C_{2, g}^{\left(n_{l}+1\right), 1}(z)+T_{f}\left(z^{2}+(1-z)^{2}\right) \ln \frac{Q^{2}}{m_{c}^{2}},
$$

where the overall factor 2 is due to the fact that the ZM coefficient function, Eq. (24), has been defined for a single quark, while in Eq. (16) the gluon coefficient function accounts for the production of two quarks ( $s$ and $\bar{c}$ ). Note also the presence of the usual collinear logarithm. For the quark piece we find

$$
C_{2, h}^{\left(n_{l}, 0\right), 1}(z)=C_{2, h}^{\left(n_{l}+1\right), 1}(z),
$$

without any collinear logarithm.

The definition of the heavy CC structure function in the ZM scheme is not unique: here we define it as the contribution to the structure function which includes all contributions to the inclusive structure function which survive when all CKM elements but $\left|V_{\mathrm{cs}}\right|$ are set to zero. With this definition, both the leading-order processes $c W^{+} \rightarrow s$ and $\bar{c} W^{+} \rightarrow \bar{s}$ contribute to it (see Fig. 7). This definition coincides with the experimental one because the struck charm antiquark must be accompanied by an (observed) charm quark, and it is free of mass singularities. The gluon initiated NLO contributions remain those shown in Fig. 6. The structure function in the massless scheme above charm threshold is then given by

$$
\begin{aligned}
F_{2, c}^{\left(n_{l}+1\right), \mathrm{CC}}\left(x, Q^{2}\right)= & 2 x\left(s\left(x, Q^{2}\right)+\bar{c}\left(x, Q^{2}\right)\right) \\
& +2 x \frac{\alpha_{s}\left(Q^{2}\right)}{2 \pi}\left\{\int _ { x } ^ { 1 } \frac { d z } { z } \left[C _ { 2 , h } ^ { ( n _ { l } + 1 ) , 1 } ( z , Q ^ { 2 } , \lambda ) \left(s\left(\frac{x}{z}, Q^{2}\right)\right.\right.\right. \\
& \left.\left.\left.+\bar{c}\left(\frac{x}{z}, Q^{2}\right)\right)+2 C_{2, g}^{\left(n_{l}+1\right), 1}\left(z, Q^{2}, \lambda\right) g\left(\frac{\xi}{z}, Q^{2}\right)\right]\right\} .
\end{aligned}
$$

The ZM-VFN massless coefficient functions have been defined in Eqs. (23)-(24). Note the factor two in front of the gluon coefficient function, to account for the production of two quarks in the two NLO subprocesses of Fig. 6.

Finally, the various schemes can be combined to construct the FONLL-A structure functions. As in the NC case, we define the FONLL structure function as follows: 


$$
\begin{aligned}
& F_{2, c}^{(\mathrm{FONLL}), \mathrm{CC}}\left(x, Q^{2}\right) \equiv F_{2, c}^{\left(n_{l}\right), \mathrm{CC}}\left(x, Q^{2}\right)+\theta\left(Q^{2}-m_{c}^{2}\right)\left(1-\frac{m_{c}^{2}}{Q^{2}}\right)^{2} F_{2, c}^{(\mathrm{d}), \mathrm{CC}}\left(x, Q^{2}\right), \\
& F_{2, c}^{(\mathrm{d}), \mathrm{CC}}\left(x, Q^{2}\right)=F_{2, c}^{\left(n_{l}+1\right), \mathrm{CC}}\left(x, Q^{2}\right)-F_{2, c}^{\left(n_{l}, 0\right), \mathrm{CC}}\left(x, Q^{2}\right),
\end{aligned}
$$

where as in the case of neutral currents we use the damping factor as default threshold prescription.

Using the explicit expressions derived in the previous section for the difference between the ZM and FFNS0 coefficient functions, Eqs. (26) and (25), we can write the difference term as

$$
F_{2, c}^{(\mathrm{d}), \mathrm{CC}}=2 x \bar{c}\left(x, Q^{2}\right)-2 x \frac{\alpha_{s}}{2 \pi} \ln \frac{Q^{2}}{m_{c}^{2}} \int_{x}^{1} \frac{d z}{z} T_{f}\left(z^{2}+(1-z)^{2}\right) g\left(\frac{x}{z}, Q^{2}\right)+\mathcal{O}\left(\alpha_{s}^{2}\right),
$$

where we have used the fact that the heavy quark distribution is $\mathcal{O}\left(\alpha_{S}\right)$. Now, it is easy to see explicitly that, in the region where $L \equiv \ln Q^{2} / m_{c}^{2}$ is not large, the "difference" term is of order $\mathcal{O}\left(\alpha_{s}^{2}\right)$ : to first order in $\alpha_{s}$ the FONLL expression coincides with the massive-scheme one also for charged current scattering. The use of the leading-order QCD evolution equations immediately leads to

$$
c\left(x, Q^{2}\right)=\bar{c}\left(x, Q^{2}\right)=\frac{\alpha_{s}\left(Q^{2}\right)}{2 \pi} \ln \frac{Q^{2}}{m_{c}^{2}} \int_{x}^{1} \frac{d z}{z} T_{f}\left(z^{2}+(1-z)^{2}\right) g\left(\frac{x}{z}, Q^{2}\right)+\mathcal{O}\left(\alpha_{s}^{2}\right) .
$$

Inserting this expansion in Eq. (30), it is trivial to check the explicit cancellation of the $\mathcal{O}\left(\alpha_{s}\right)$ terms, that is, that near the heavy quark threshold the difference term is of order $F_{2, c}^{(\mathrm{d}), \mathrm{CC}}=\mathcal{O}\left(\alpha_{s}^{2}\right)$.

The final FONLL-A expressions for the charged current charm production structure function $F_{2, c}^{\mathrm{CC}}$ are given by

$$
\begin{aligned}
F_{2, c}^{(\mathrm{FONLL}), \mathrm{CC}}\left(x, Q^{2}\right)= & 2 \xi s\left(\xi, Q^{2}\right)+\theta\left(Q^{2}-m_{c}^{2}\right)\left(1-\frac{m_{c}^{2}}{Q^{2}}\right)^{2} 2 x \bar{c}\left(x, Q^{2}\right) \\
& +2 \xi \frac{\alpha_{s}\left(Q^{2}\right)}{2 \pi}\left\{\int _ { \xi } ^ { 1 } \frac { d z } { z } \left[C _ { 2 , h } ^ { ( n _ { l } ) , 1 } ( z , Q ^ { 2 } , \lambda ) \left(s\left(\frac{\xi}{z}, Q^{2}\right)\right.\right.\right. \\
& \left.+\theta\left(Q^{2}-m_{c}^{2}\right)\left(1-\frac{m_{c}^{2}}{Q^{2}}\right)^{2} 2 x \bar{c}\left(\frac{\xi}{z}, Q^{2}\right)\right) \\
& \left.\left.+C_{2, g}^{\left(n_{l}\right), 1}\left(z, Q^{2}, \lambda\right) g\left(\frac{\xi}{z}, Q^{2}\right)\right]\right\} \\
& -\theta\left(Q^{2}-m_{c}^{2}\right)\left(1-\frac{m_{c}^{2}}{Q^{2}}\right)^{2} 2 x \frac{\alpha_{s}\left(Q^{2}\right)}{2 \pi} \\
& \times \int_{x}^{1} \frac{d z}{z} T_{f}\left(z^{2}+(1-z)^{2}\right) g\left(\frac{x}{z}, Q^{2}\right) .
\end{aligned}
$$

It can be easily verified that Eq. (32) reduces to the FFN scheme, Eq. (9) at the heavy quark threshold $Q^{2}=m_{c}^{2}$, and to the ZM-VFN expression (27) in the asymptotic region $Q^{2} \gg m_{c}^{2}$.

The above derivation generalizes straightforwardly to the other relevant charged current structure functions $x F_{3, c}^{\mathrm{CC}}$ and $F_{L, c}^{\mathrm{CC}}$, as well as to the case with a general CKM quark mixing matrix. 

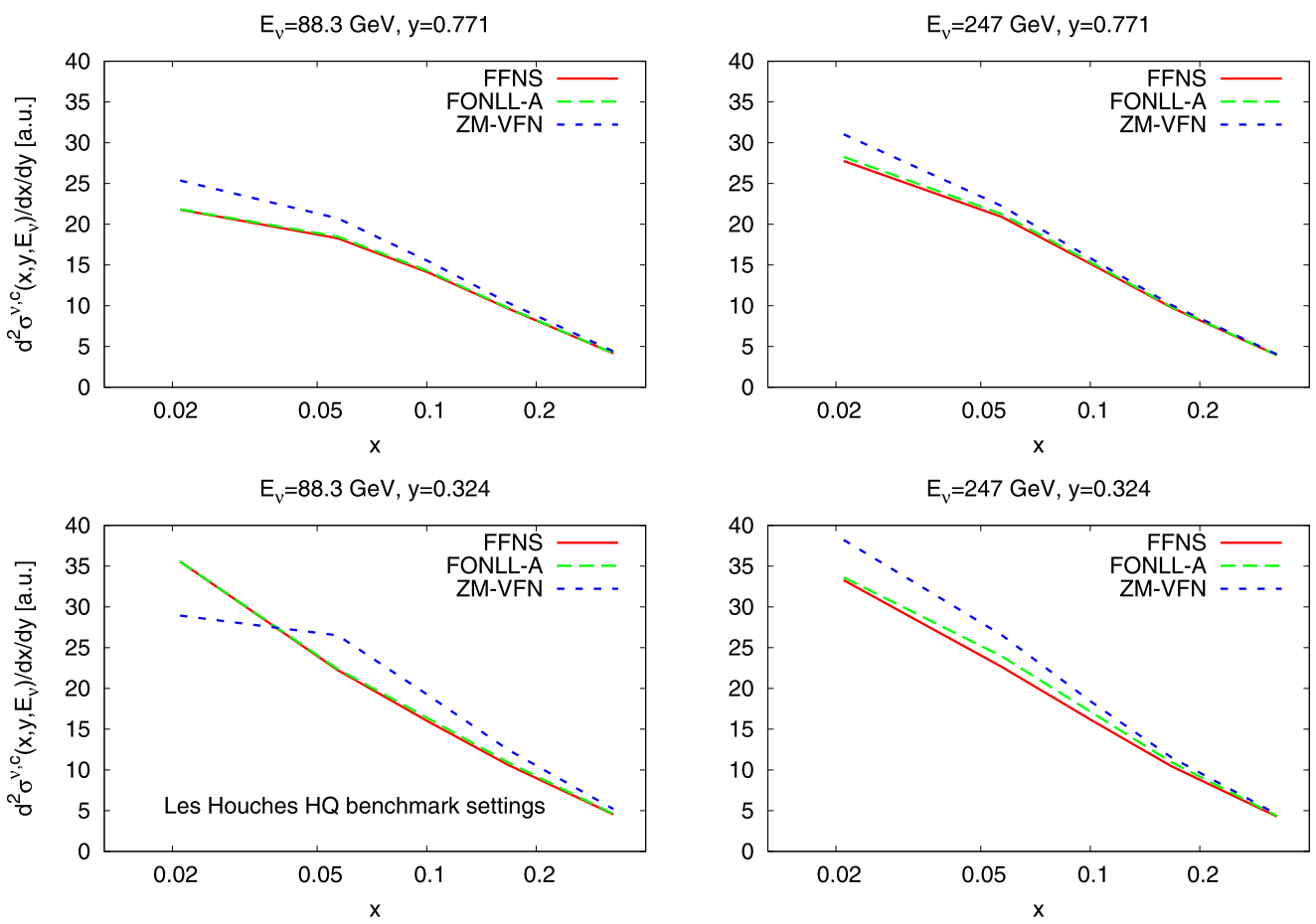

Fig. 8. Comparison of different schemes for charm production in neutrino-induced DIS. The kinematic range is representative of the NuTeV dimuon data range. We compare the ZM-VFN, FFN and FONLL-A schemes at the level of the neutrino induced charm production cross-section, Eq. (33). The settings are the same as those of the Les Houches heavy quark benchmark comparison [19].

Note that in all the results shown below the standard CKM mixing has been assumed, with the CKM matrix elements set to their PDG values [65].

Now that we have defined the FONLL-A general-mass scheme for charged current structure functions, we can compare the various schemes (ZM, FFNS, FONLL-A) in the kinematic region that is most relevant in the global PDF analysis, namely the region covered by the NuTeV dimuon measurements [66] (see Fig. 1). In Fig. 8 we show the results of such a comparison between various schemes for charm production in neutrino-induced charged current scattering. Results are compared at the level of the phenomenologically relevant charm production reduced crosssection, defined as [9]:

$$
\begin{aligned}
\tilde{\sigma}^{v(\bar{v}), c}\left(x, y, Q^{2}\right) \\
\equiv \\
=\frac{1}{E_{v}} \frac{d^{2} \sigma^{v(\bar{v}), c}}{d x d y}\left(x, y, Q^{2}\right) \\
=\frac{G_{F}^{2} M_{N}}{2 \pi\left(1+Q^{2} / M_{W}^{2}\right)^{2}}\left[\left(\left(Y_{+}-\frac{2 M_{N}^{2} x^{2} y^{2}}{Q^{2}}-y^{2}\right)\left(1+\frac{m_{c}^{2}}{Q^{2}}\right)+y^{2}\right) F_{2, c}^{v(\bar{v})}\left(x, Q^{2}\right)\right. \\
\left.\quad-y^{2} F_{L, c}^{v(\bar{v})}\left(x, Q^{2}\right) \pm Y_{-} x F_{3, c}^{v(\bar{v})}\left(x, Q^{2}\right)\right]
\end{aligned}
$$



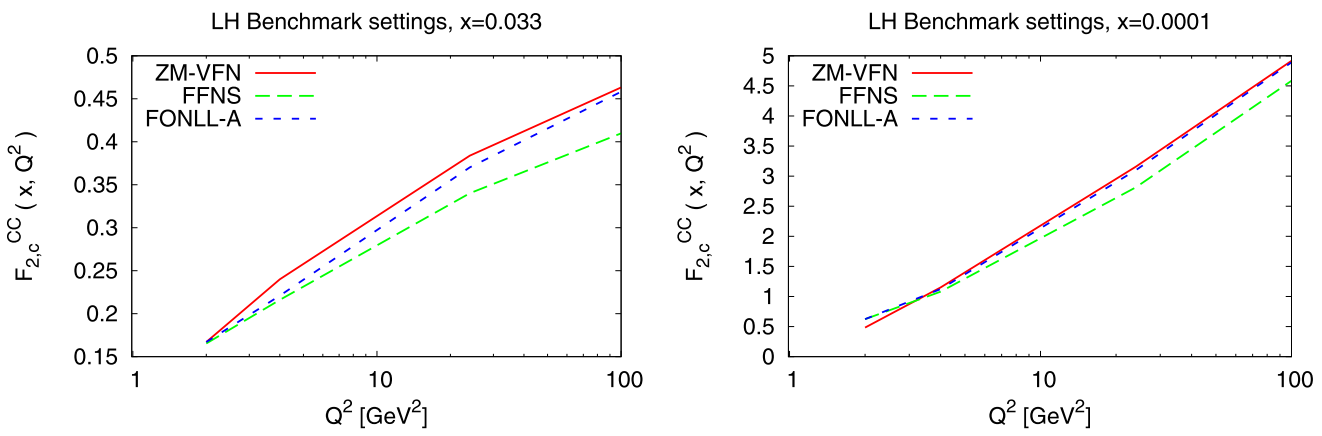

Fig. 9. Comparison of different schemes for charm production in neutrino-induced DIS. We show the $F_{2, c}^{\mathrm{CC}}$ structure function in the massless, massive and FONLL-A schemes; in this case the FONLL-A expression is given by Eq. (32). The settings are the same as those of the Les Houches heavy quark benchmark comparison [19].

with $Q^{2}=2 M_{N} E_{v} x y$ and $Y_{ \pm}=1 \pm(1-y)^{2}$. In Fig. 8 we compare the various schemes in some representative bins of the NuTeV dimuon kinematics [66]. PDFs and other settings are those of the Les Houches heavy quark benchmark comparison [19]. We observe that in the kinematic region of neutrino data (both inclusive CHORUS data and dimuon NuTeV data), the FONLLA result is very close to the FFN scheme computation, and it only begins to differ from it at the highest energies, where resummation of charm mass collinear logarithms begins to become relevant.

Even if the differences between the FFN and FONLL-A schemes for charged current scattering in the NuTeV kinematic region are moderate, as shown in Fig. 8, they become rather more important at small- $x$ and medium-large- $Q^{2}$, where the charm and gluon PDFs become larger. To illustrate this, in Fig. 9 we compare the charged current charm structure function $F_{2, c}^{\mathrm{CC}}$ as a function of $Q^{2}$ for two different values of $x$. Notice in particular that at very small- $x$ the FONLLA expression is essentially the massless result. However, producing dimuons at $x \sim 10^{-3}$ and $Q^{2} \sim 10 \mathrm{GeV}^{2}$, where differences are larger, requires a fixed target neutrino experiment with a neutrino beam with energy in the multi- $\mathrm{TeV}$ range, which is not foreseen in the near future. Therefore one can conclude that any reasonable general-mass scheme for charged current scattering will be very close to the FFNS in the region of experimental data.

The FONLL-A calculation of charged current structure functions has been implemented in an $x$-space code, FONLLdisCC, that we will use for benchmarking purposes. This is the analogue of the FONLLdis code for neutral currents [67], however is rather simpler since the unknown $\mathcal{O}\left(\alpha_{s}^{2}\right)$ massive coefficient functions do not have to be implemented. Our implementation of the FFNS calculations has been benchmarked with the corresponding results of the MSTW08 code [68], finding perfect agreement. We have also compared the FONLL-A and MSTW08 general-mass schemes for charged currents, finding qualitative agreement but some quantitative differences. A detailed comparison between different general-mass schemes for charged current structure functions, analogous to the Les Houches benchmarks for neutral current structure functions [19] is still missing and would be highly desirable.

\section{Results}

In this section we present the NNPDF2.1 parton determination. First, we discuss the statistical features of the fit, then we turn to a comparison of NNPDF2.1 PDFs and uncertainties with other 
Table 3

Table of statistical estimators for NNPDF2.1 with $N_{\text {rep }}=$ 1000 replicas. The total average uncertainty is given in percentage. All the $\chi^{2}$ and $E$ values have been computed using the same $t_{0}$ covariance matrix [69] used for minimization.

\begin{tabular}{ll}
\hline$\chi_{\text {tot }}^{2}$ & 1.16 \\
$\langle E\rangle \pm \sigma_{E}$ & $2.24 \pm 0.09$ \\
$\left\langle E_{\mathrm{tr}}\right\rangle \pm \sigma_{E_{\mathrm{tr}}}$ & $2.22 \pm 0.11$ \\
$\left\langle E_{\mathrm{val}}\right\rangle \pm \sigma_{E_{\mathrm{val}}}$ & $2.28 \pm 0.12$ \\
$\langle\mathrm{TL}\rangle \pm \sigma_{\mathrm{TL}}$ & $(1.6 \pm 0.6) \times 10^{4}$ \\
$\left\langle\chi^{2(k)}\right\rangle \pm \sigma_{\chi^{2}}$ & $1.25 \pm 0.09$ \\
$\left\langle\sigma^{(\mathrm{exp})}\right\rangle_{\text {dat }}(\%)$ & $11.3 \%$ \\
$\left\langle\sigma^{(\mathrm{net})}\right\rangle_{\text {dat }}(\%)$ & $4.4 \%$ \\
$\left\langle\rho^{(\mathrm{exp})}\right\rangle_{\text {dat }}$ & 0.18 \\
$\left\langle\rho^{(\mathrm{net})}\right\rangle_{\text {dat }}$ & 0.56 \\
\hline
\end{tabular}

Table 4

Same as Table 3 for individual experiments. All estimators have been obtained with $N_{\text {rep }}=1000$ replicas. Note that experimental uncertainties are always given in percentage. In the second and third columns the NNPDF2.1 and NNPDF2.0 sets [10] $\chi^{2}$ have been computed with the $t_{0}$ prescription.

\begin{tabular}{llllllll}
\hline Experiment & $\chi^{2}$ & $\chi_{2.0}^{2}$ & $\langle E\rangle$ & $\left\langle\sigma^{(\exp )}\right\rangle_{\text {dat }}(\%)$ & $\left\langle\sigma^{(\text {net })}\right\rangle_{\text {dat }}(\%)$ & $\left\langle\rho^{(\exp )}\right\rangle_{\text {dat }}$ & $\left\langle\rho^{(\text {net })}\right\rangle_{\text {dat }}$ \\
\hline NMC-pd & 0.97 & 1.04 & 2.04 & $1.9 \%$ & $0.5 \%$ & 0.03 & 0.37 \\
NMC & 1.73 & 1.73 & 2.79 & $5.0 \%$ & $1.5 \%$ & 0.16 & 0.71 \\
SLAC & 1.27 & 1.42 & 2.34 & $4.4 \%$ & $1.6 \%$ & 0.31 & 0.79 \\
BCDMS & 1.28 & 1.30 & 2.33 & $5.7 \%$ & $2.3 \%$ & 0.47 & 0.60 \\
HERAI-AV & 1.07 & 1.15 & 2.15 & $2.5 \%$ & $1.2 \%$ & 0.06 & 0.35 \\
CHORUS & 1.15 & 1.24 & 2.23 & $15.1 \%$ & $4.7 \%$ & 0.08 & 0.32 \\
FLH108 & 1.37 & 1.50 & 2.36 & $72.0 \%$ & $4.0 \%$ & 0.64 & 0.67 \\
NTVDMN & 0.76 & 0.73 & 1.77 & $21.1 \%$ & $14.1 \%$ & 0.04 & 0.62 \\
ZEUS-H2 & 1.29 & 1.33 & 2.32 & $13.4 \%$ & $1.2 \%$ & 0.27 & 0.51 \\
ZEUSF2C & 0.78 & - & 1.80 & $23.3 \%$ & $3.1 \%$ & 0.08 & 0.41 \\
H1F2C & 1.50 & - & 2.52 & $17.3 \%$ & $3.0 \%$ & 0.30 & 0.40 \\
DYE605 & 0.84 & 0.87 & 1.92 & $22.3 \%$ & $7.9 \%$ & 0.47 & 0.76 \\
DYE866 & 1.27 & 1.29 & 2.37 & $20.1 \%$ & $9.2 \%$ & 0.20 & 0.52 \\
CDFWASY & 1.86 & 1.84 & 3.08 & $6.0 \%$ & $4.4 \%$ & 0.51 & 0.75 \\
CDFZRAP & 1.65 & 1.85 & 2.80 & $11.5 \%$ & $3.6 \%$ & 0.82 & 0.72 \\
D0ZRAP & 0.60 & 0.60 & 1.62 & $10.2 \%$ & $4.1 \%$ & 0.53 & 0.76 \\
CDFR2KT & 0.97 & 1.01 & 2.10 & $22.2 \%$ & $4.5 \%$ & 0.78 & 0.57 \\
D0R2CON & 0.84 & 0.86 & 1.92 & $16.8 \%$ & 0.77 & 0.59 \\
\hline
\end{tabular}

PDF determinations and with previous NNPDF releases. A detailed comparison between the NNPDF2.1 and 2.0 sets follows, in which we discuss one by one the impact of the differences between the two fits, due both to the choice of dataset and to the different theoretical framework. The implications of the NNPDF2.1 set for LHC observables are discussed in the next sections.

\subsection{Statistical features}

Statistical estimators for the NNPDF2.1 fit are shown in Table 3 for the global fit and in Table 4 for individual experiments. In Table 4 the $\chi^{2}$ values for NNPDF2.0 are also shown for 
comparison. As in Ref. [10], $\chi_{\text {tot }}^{2}$ is computed comparing the central (average) NNPDF2.1 fit to the original experimental data, $\left\langle\chi^{2(k)}\right\rangle$ is computed comparing to the data each NNPDF2.1 replica and averaging over replicas, while $\langle E\rangle$ is the quantity which is minimized, i.e. it coincides with the $\chi^{2}$ computed comparing each NNPDF2.1 replica to the data replica it is fitted to, with the three values given corresponding to the total, training and validation data sets. It is important to observe that all values of $\chi^{2}$ shown in Tables 3-4 are obtained using the covariance matrix with normalization uncertainties included according to the $t_{0}$ method of Ref. [69] (also given as Eq. (1) in Ref. [10]). In Tables 9-10 of Ref. [10] all values of $\chi_{\text {tot }}^{2}$ and $\left\langle\chi^{2(k)}\right\rangle$ were instead given with $\chi^{2}$ defined using the "standard" covariance matrix (given e.g. in Eq. (52) of that reference), which includes normalization uncertainties less accurately than the $t_{0}$ covariance matrix. This was done in order to ease comparison between the results of Ref. [10] and the NNPDF1.x PDFs, in which the $t_{0}$ method was not yet used and normalization uncertainties were not fully accounted for. The values of $\chi_{\text {tot }}^{2}$ for the NNPDF2.0 shown here in Tables 3-4 have been recomputed using the $t_{0}$ covariance matrix in order to ease comparison with NNPDF2.1 and with other PDF determinations which also include normalization uncertainties albeit with various other methods. The value of $\chi_{\text {tot }}^{2}$ for the NNPDF2.0 global fit computed using the $t_{0}$ method, to be compared to the NNPDF2.1 value of Table 3 , is $\chi_{\text {tot }}^{2}=1.23$ (very close to the value $\chi_{\text {tot }}^{2}=1.21$ of Table 9 in Ref. [10], computed with the "standard" covariance matrix).

The NNPDF2.1 PDF fit has the following noticeable features:

- The quality of the global fit as measured by the value $\chi^{2}=1.16$ is rather better than for the NNPDF2.0 fit without heavy quark mass effects.

- As compared to the NNPDF2.0 results, the quality of the fit to all datasets improves or remains similar. The most noticeable improvements can be found for the HERA-I average dataset and for CHORUS. The improvement in the description of HERA data arises both from the improved heavy flavor treatment and the more conservative kinematic cuts.

- An excellent description of the combined HERA-I inclusive data, $\chi^{2}=1.07$, is obtained. Similarly, a reasonable description of the HERA charm structure function data is achieved.

- The quality of the fit to hadronic data is not affected by the use of the FONLL-A GM scheme for deep-inelastic observables, as it can be seen by comparing the second and third column of Table 4 .

The distribution of $\chi^{2(k)}, E_{\mathrm{tr}}^{(k)}$ and training lengths among the $N_{\text {rep }}=1000$ NNPDF2.1 replicas are shown in Figs. 10 and 11 respectively. While most of the replicas fulfill the stopping criterion, a fraction $(\sim 12 \%)$ of them stops at the maximum training length $N_{\text {gen }}^{\max }$ which has been introduced in order to avoid unacceptably long fits. This causes some loss of accuracy for outliers fits (i.e. those in the tail of the distribution): we have checked that as $N_{\text {gen }}^{\max }$ is raised more and more of these replicas stop, and that the loss of accuracy due to this choice of value of $N_{\text {gen }}^{\max }$ is reasonably small, in that the features of the global fit change very little if $N_{\text {gen }}^{\max }$ is raised.

It is instructive to compare the quality of the fit with the corresponding results obtained in the recent CT10 analysis. ${ }^{6}$ In Table 5 we compare the $\chi^{2}$ of the common sets in NNPDF2.1 and CT10, along with the number of data points in each fit (which differ because of different kinematic cuts, see Table 1). It should be borne in mind that the $\chi^{2}$ is defined in a somewhat different way by the CTEQ/CT group, specifically, but not only, in what concerns the treat-

\footnotetext{
6 We thank Pavel Nadolsky for providing us with these numbers.
} 

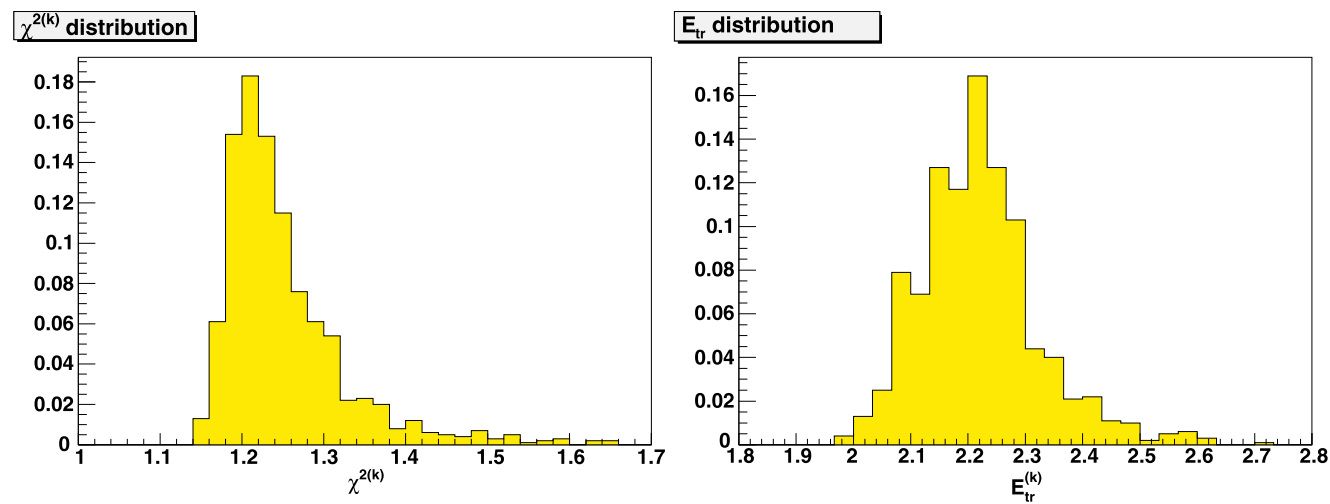

Fig. 10. Distribution of $\chi^{2(k)}$ (left) and $E_{\text {tr }}^{(k)}$ (right), over the sample of $N_{\text {rep }}=1000$ replicas.

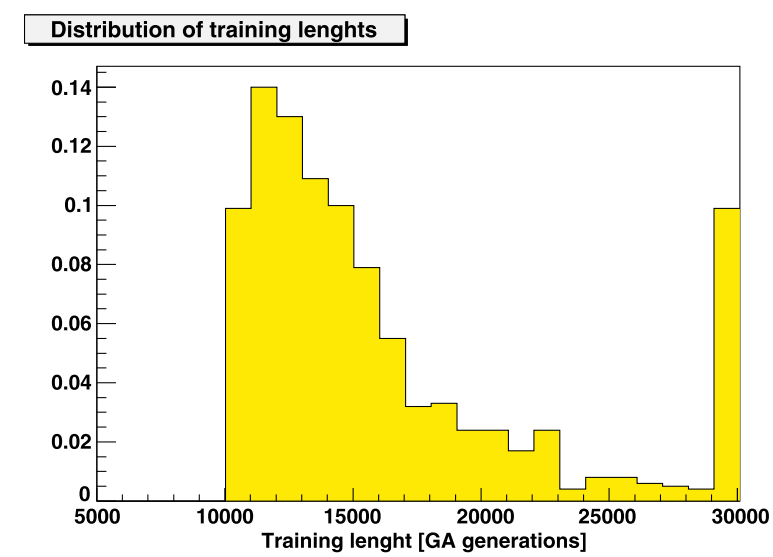

Fig. 11. Distribution of training lengths over the sample of $N_{\text {rep }}=1000$ replicas.

ment of normalization errors (see Ref. [16]): hence this comparison should be taken with care. From this comparison, we can see that the two sets have a comparable fit quality to fixed target DIS, CT10 being somewhat better for BCDMS proton and NNPDF2.1 rather better for NMC deuteron/proton ratio. The fit to HERA-I and Tevatron jet data is rather better in NNPDF2.1. Comparable fit quality to the Drell-Yan and vector boson production data is obtained in the two cases, with somewhat smaller $\chi^{2}$ in the CT10 fit. No comparison is attempted for the HERA $F_{2}^{c}$ data because of the very different kinematic cuts used in the two fits. A similar comparison to MSTW08 would be less significant because in the MSTW08 fit correlated systematics are not included in the covariance matrix for some datasets.

\subsection{Parton distributions}

The NNPDF2.1 PDFs are compared to the previous NNPDF2.0 PDFs in Figs. 12 (singlet sector) and Fig. 13 (non-singlet sector). 
Table 5

Comparison of $\chi^{2}$ per data point for experiments which are common to the NNPDF2.1 and CT10 PDF determinations. For each PDF set the number of data points obtained with the kinematic cuts of Table 1 is given.

\begin{tabular}{lcccc}
\hline Experiment & \multicolumn{2}{l}{ NNPDF2.1 } & \multicolumn{2}{c}{ CT10 } \\
\cline { 2 - 4 } & $N_{\text {dat }}$ & $\chi^{2}$ & $N_{\text {dat }}$ & 121 \\
\hline NMC-pd & 132 & 0.97 & 196 & 1.28 \\
NMC & 221 & 1.73 & 337 & 1.71 \\
BCDMSp & 333 & 1.28 & 250 & 1.14 \\
BCDMSd & 248 & 1.15 & 579 & 1.12 \\
HERAI-AV & 592 & 1.07 & 38 & 1.17 \\
NTVnuDMN & 41 & 0.50 & 33 & 0.94 \\
NTVnbDMN & 38 & 0.42 & 119 & 0.91 \\
DYE605 & 119 & 0.85 & 184 & 0.81 \\
DYE866p & 184 & 1.31 & 15 & 1.21 \\
DYE866r & 15 & 0.77 & 29 & 0.64 \\
CDFZRAP & 29 & 1.62 & 28 & 1.44 \\
D0ZRAP & 28 & 0.59 & 76 & 0.54 \\
CDFR2KT & 76 & 0.97 & 110 & 1.55 \\
D0R2CON & 110 & 0.84 & & 1.13 \\
\hline
\end{tabular}
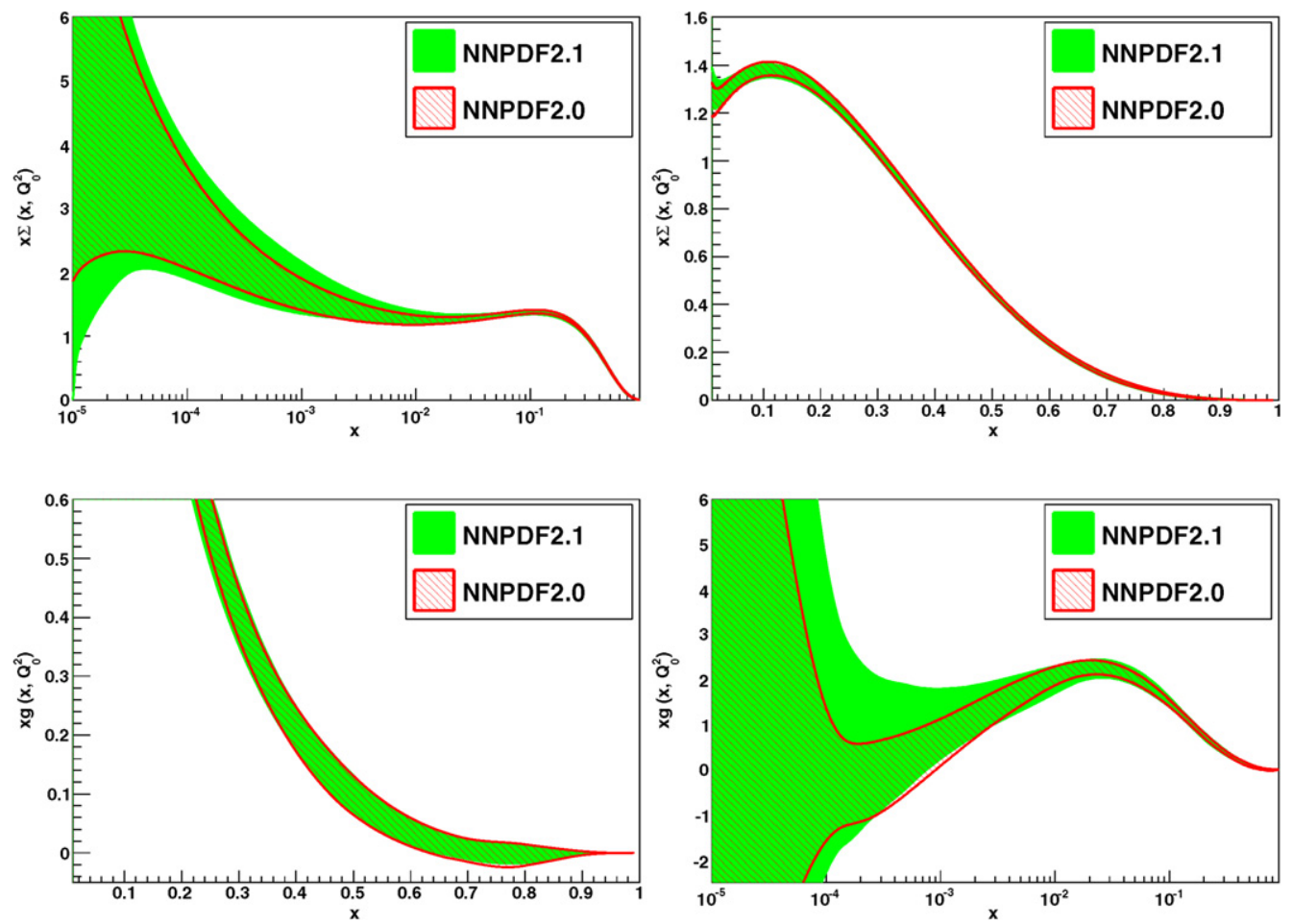

Fig. 12. Comparison of NNPDF2.1 and NNPDF2.0 singlet sector PDFs, computed using $N_{\text {rep }}=1000$ replicas from both sets. All error bands shown correspond to one- $\sigma$. 

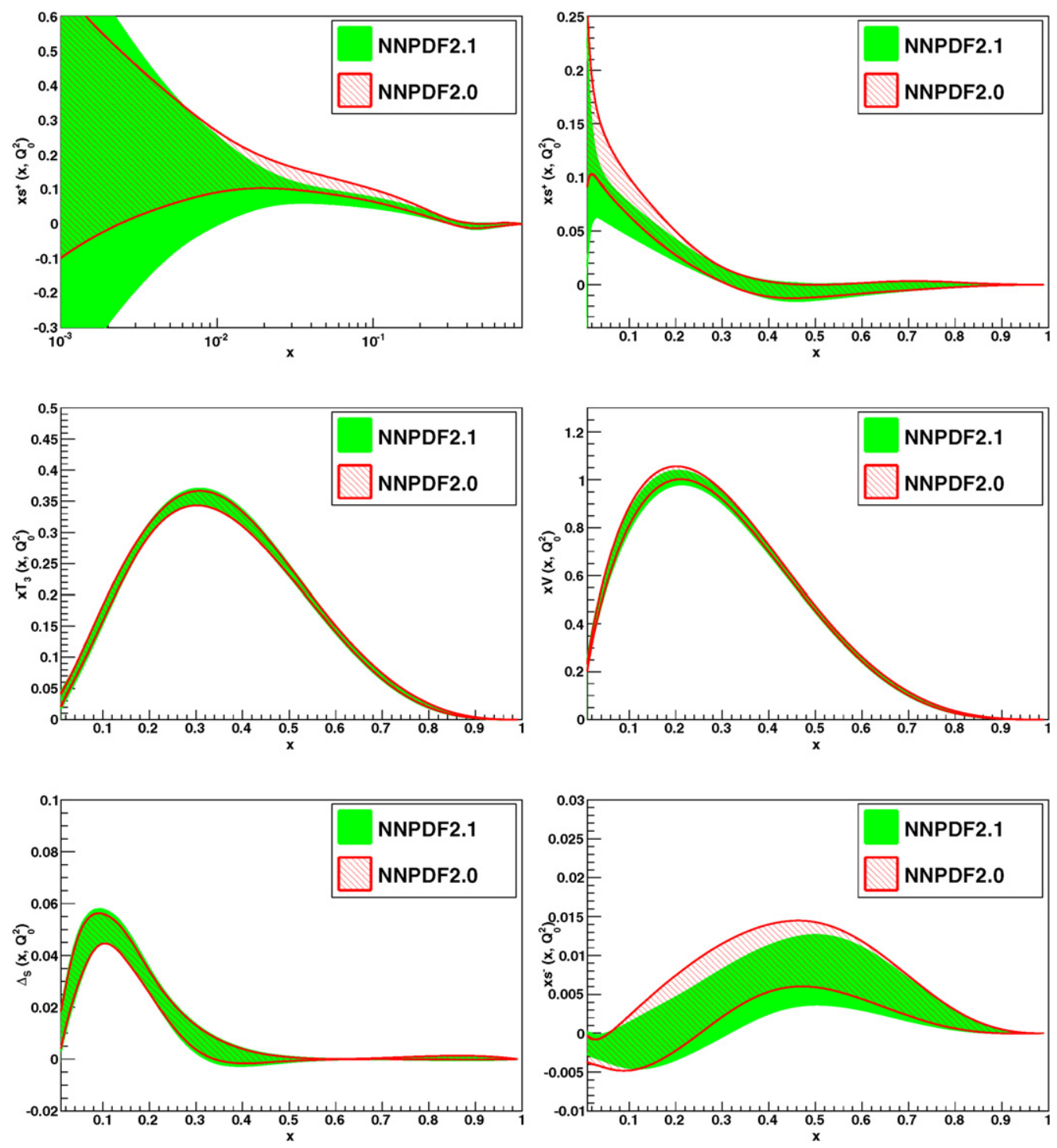

Fig. 13. Same as Fig. 12 for the non-singlet sector PDFs.

- The singlet PDF at medium and small- $x$ is rather similar in the two cases, but it is somewhat larger in the NNPDF2.1 set.

- Thanks to the new positivity constraint on $F_{2}^{c}$ in NNPDF2.1, the gluon remains always positive even at the largest values of $x$, where occasionally went very slightly negative in NNPDF2.0.

- In NNPDF2.1 the small- $x$ gluon is larger than in NNPDF2.0. We will show that this arises from the use of a GM scheme as compared to the ZM scheme in NNPDF2.0. Also, the medium and small- $x$ gluon has a somewhat larger uncertainty in NNPDF2.1 as compared to NNPDF2.0. We will show below that this uncertainty increase is due to the new kinematic cut. 

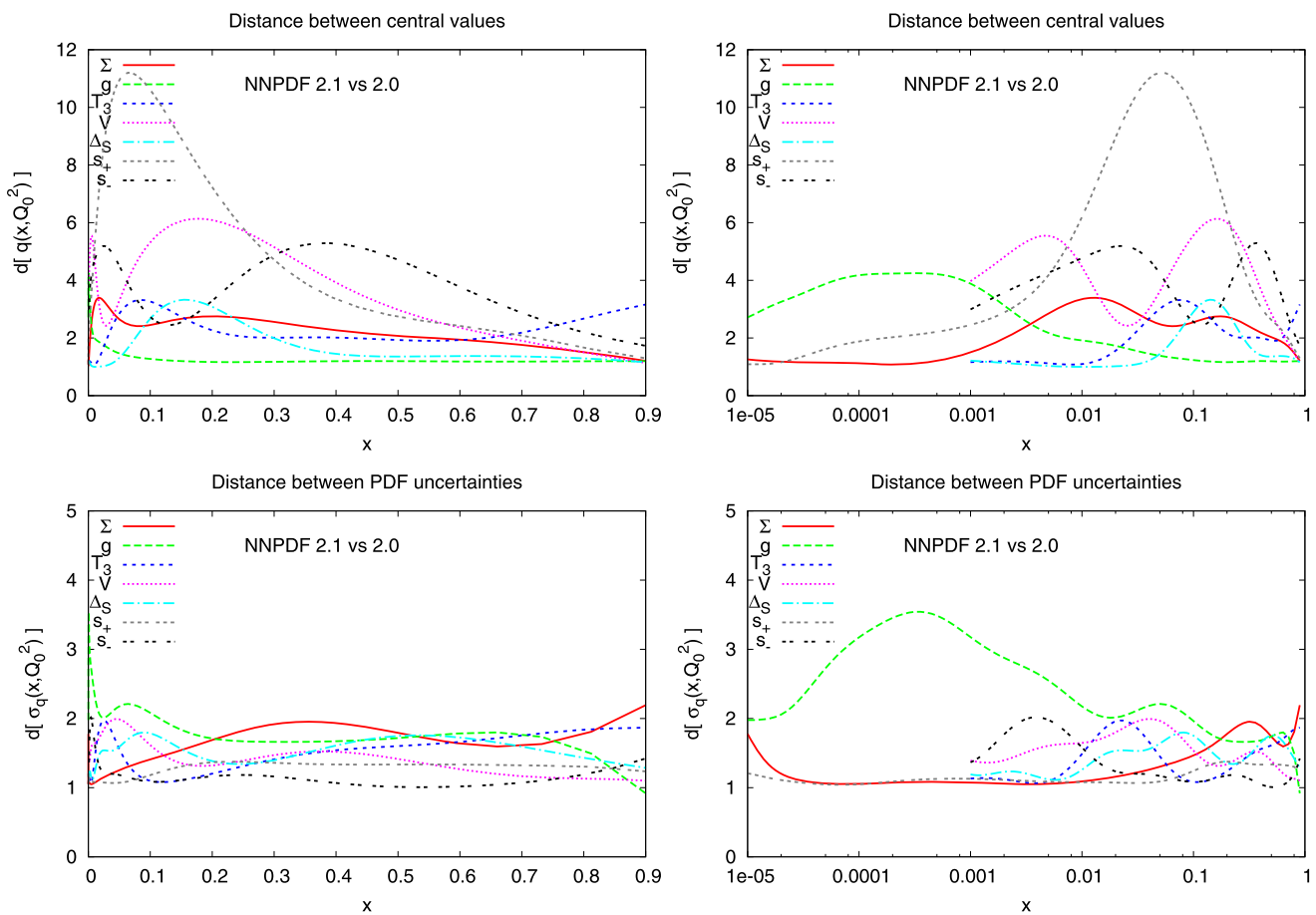

Fig. 14. Distance between the NNPDF2.0 and NNPDF2.1 parton sets. All distances are computed from sets of $N_{\text {rep }}=100$ replicas.

- As expected differences in the large- $x$ valence PDFs are rather modest. The GM scheme does not affect the large- $x$ PDFs but the cross-talk induced by the sum rules and other constraints induces small modifications also in the valence sector, always well below the one- $\sigma$ level.

- The strange PDF in NNPDF2.1 is somewhat smaller than in NNPDF2.0. This may appear surprizing as the main expected effect of the inclusion of heavy quark mass effects is a suppression of charm which then leads to an enhancement of all other PDFs. However, in NNPDF2.0 for dimuon data (which have a sizable impact on strangeness) instead of a pure zero-mass scheme, the so-called improved zero-mass (IZM) approximation of Ref. [55] was used to approximate heavy quark mass effects. It turns out that this IZM method actually overestimates heavy quark mass effects, thus leading to a slight over-suppression of charm in NNPDF2.0. We will check explicitly below (see Fig. 8) that when comparing NNPDF2.1 to a pure zero-mass fit strangeness is somewhat enhanced as one would expect.

- We see from Fig. 13 that the strange asymmetry in NNPDF2.1 is very close to that of NNPDF2.0. An important result of the NNPDF2.0 analysis was that the strange asymmetry $s^{-}\left(x, Q^{2}\right)$ was of the proper size to completely cancel the so-called $\mathrm{NuTeV}$ anomaly with rather reduced uncertainties. It is clear that this holds true also with the updated NNPDF2.1 set, confirming the results of the analysis of [9] that showed that heavy quark mass effects have a very moderate impact in the determination of the strangeness asymmetry. The implications for the NuTeV anomaly will be discussed in Section 5.4. 

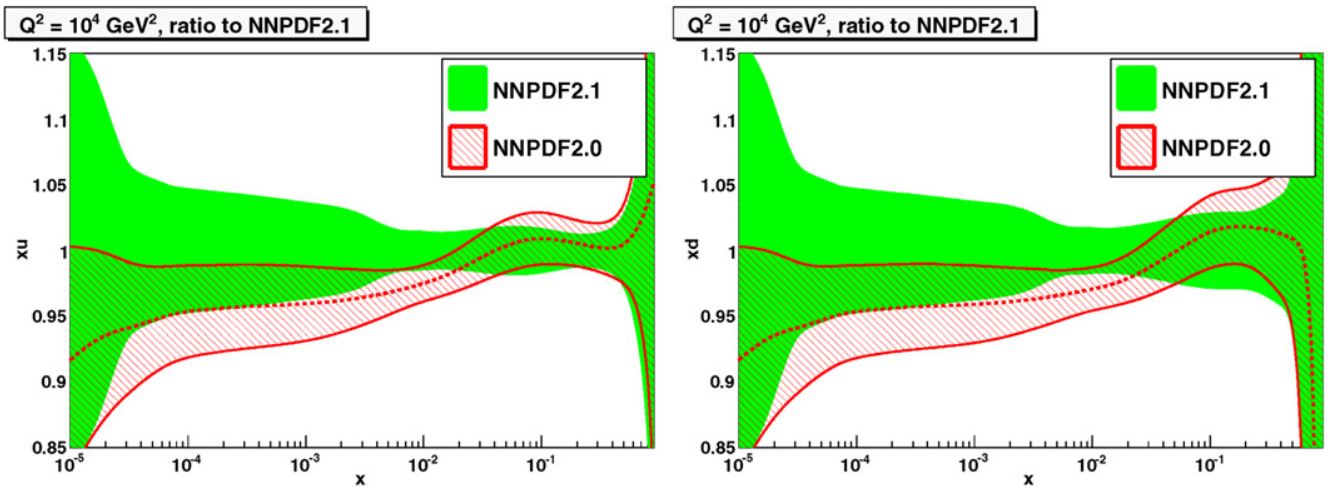

$\mathrm{Q}^{2}=10^{4} \mathrm{GeV}^{2}$, ratio to NNPDF2.1
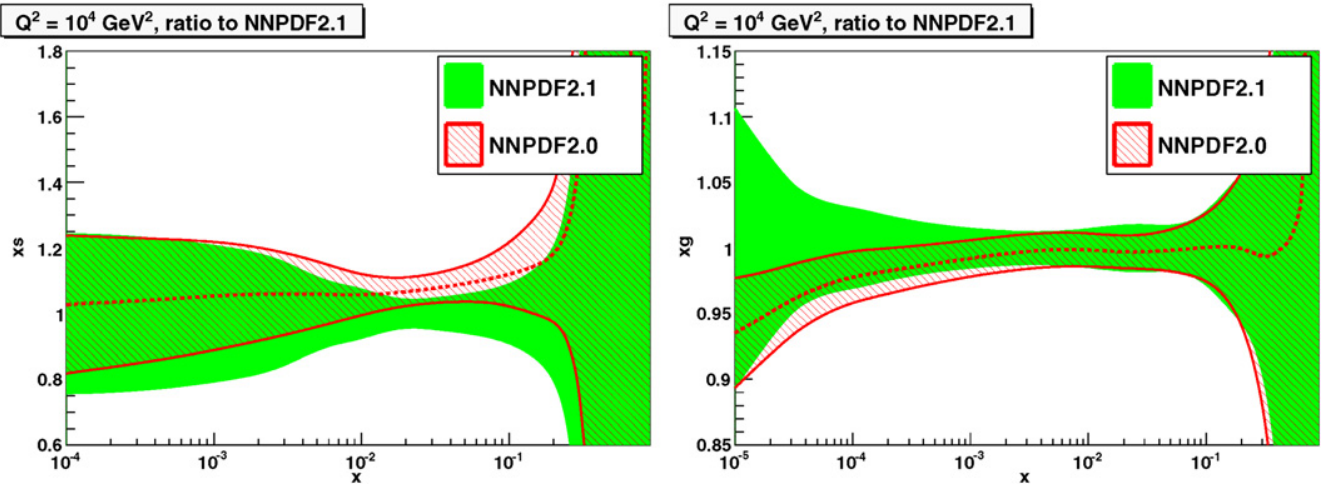

Fig. 15. Comparison between NNPDF2.1 and NNPDF2.0 light quark and gluon PDFs at $Q^{2}=10^{4} \mathrm{GeV}^{2}$. All curves are shown as ratios to the central NNPDF2.1 result.

The comparison between NNPDF2.0 and NNPDF2.1 is further quantified by the computation of the distance [10] between the two sets, shown in Fig. 14. Note that $d \sim 1$ corresponds to two sets of replicas which come from the same underlying probability distribution, while (using $N_{\text {rep }}=100$ replicas) $d \sim 7$ corresponds to a one- $\sigma$ difference (see Appendix A of Ref. [10]). One concludes that while clearly the two sets do not come from the same underlying distributions, all PDFs but the strange are consistent at the one- $\sigma$, and even the strangeness is consistent at the $90 \%$ confidence level. The largest differences are seen in the medium- $x$ strangeness and to a lesser extent in the medium and small- $x$ gluon.

The differences between NNPDF2.0 and NNPDF2.1 PDFs at the initial scale displayed in Figs. 12-14, once evolved up to the $W$ and $Z$ scale, are sufficient to lead to differences between gluon and light sea quark distributions up to the one- $\sigma$ level at small- $x$, as shown in Fig. 15 where we plot the NNPDF2.0/NNPDF2.1 ratio for individual light flavors and the gluon at $Q^{2}=$ $10^{4} \mathrm{GeV}^{2}$.

The NNPDF2.1 PDFs are compared to the other global PDF sets CT10 [16] and MSTW08 [17] in Figs. 16-17, which is interesting to contrast to the analogous plot which in Ref. [10] (Figs. 1819 of that reference) compared the NNPDF2.0, CTEQ6.6 and MSTW08 PDF sets.

- The general agreement of the gluon in the medium-/small- $x$ region is improved, both because the central value of NNPDF2.1 is now in better agreement with MSTW08 (most likely due to the inclusion of heavy quark mass effects) and also because the CT10 central value and 

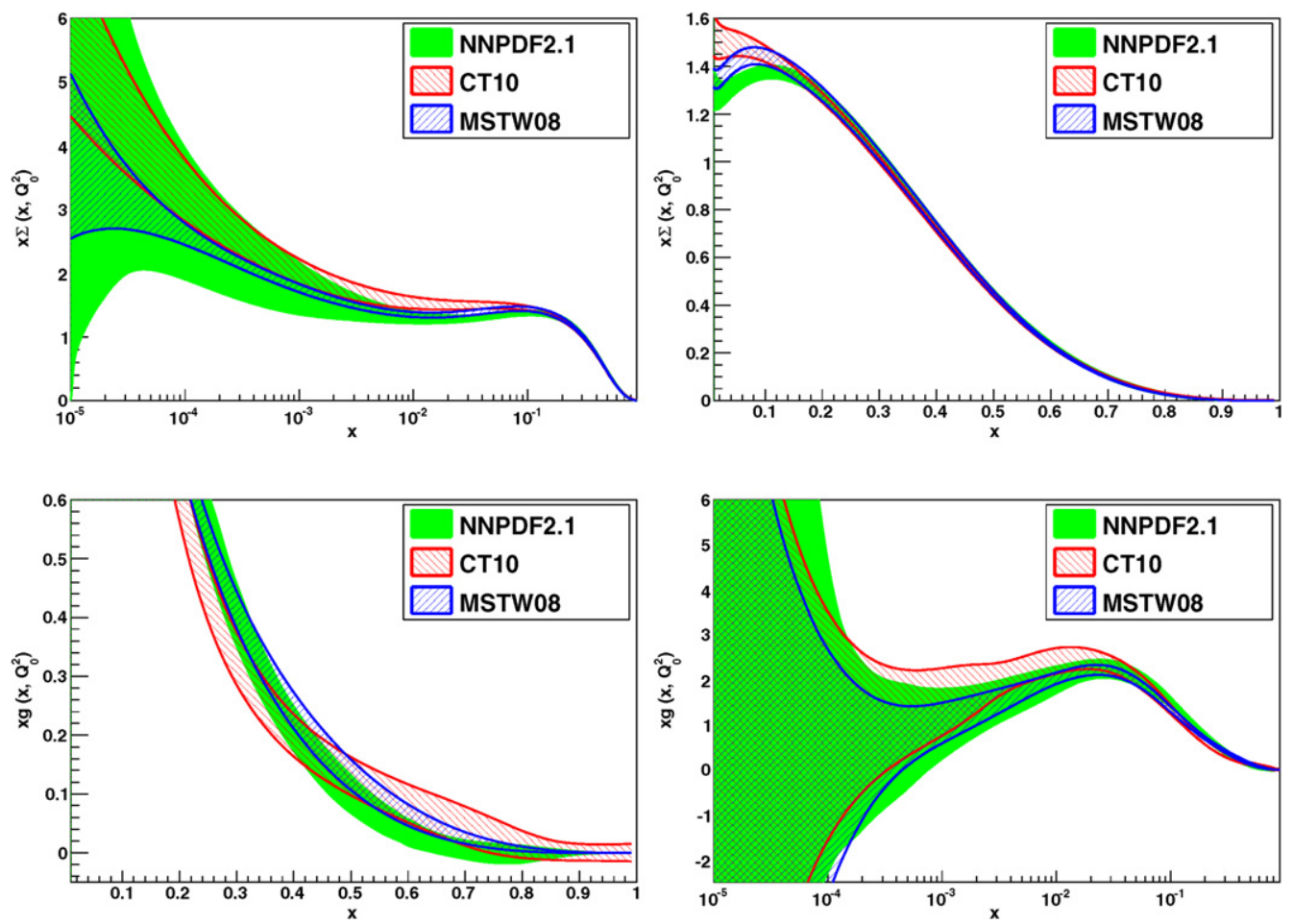

Fig. 16. The NNPDF2.1 singlet sector PDFs, compared with the CT10 and MSTW08 PDFs. The results for NNPDF2.1 have been obtained with $N_{\text {rep }}=1000$ replicas. All PDF errors are given as one- $\sigma$ uncertainties.

especially uncertainty are in much better agreement with the wider NNPDF and MSTW uncertainties, due to the use of a more flexible gluon parametrization in CT10 with respect to CTEQ6.6. The large- $x$ gluon however is in marginal agreement.

- The small changes in valence and triplet distributions between NNPDF2.0 and NNPDF2.1 go anyway in the direction of improving the agreement with the other global sets.

- The strange PDFs are quite different, presumably due to the fact that a much less flexible parametrization is adopted by CT/CTEQ and MSTW in comparison to NNPDF.

- The medium- $x$ singlet is in marginal agreement. This may be a byproduct of the poor agreement in strangeness.

The effect on LHC observables will be discussed in Section 5, where we will show that even though there is generally a reasonable agreement between global sets, there remain some significant differences, mostly related to the rather different large- $x$ gluon in CT10 as shown in Fig. 16.

\subsection{Detailed comparison to NNPDF2.0: theoretical framework and dataset}

We now assess the separate impact of the inclusion of heavy quark mass effects and of the charm structure function data on the NNPDF2.1 PDF determination. 

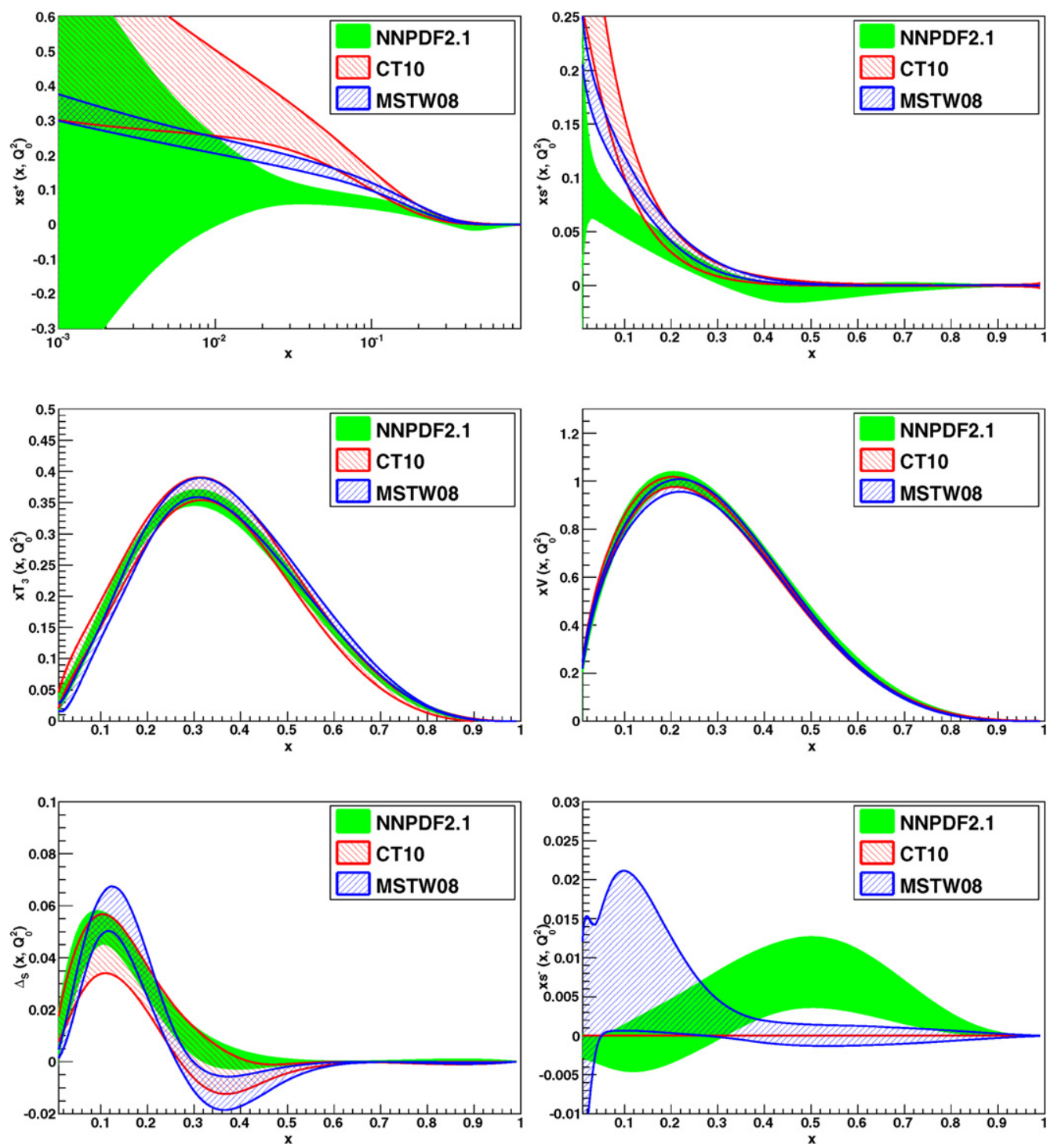

Fig. 17. Same as Fig. 16 for the non-singlet sector PDFs.

First, we must discuss the impact of raising the kinematic cut $Q_{\min }^{2}$ within the ZM scheme; then we compare the ZM and GM fits; next we investigate the impact of including the HERA charm structure function data into the NNPDF2.1 analysis; finally we estimate the impact of ambiguities related to the treatment of heavy quarks. In each case, we will show the distances between PDFs as well as the PDFs that are most affected by each step.

- New kinematic cut $Q_{\text {min }}^{2}$

In Fig. 18 we show the distance between NNPDF2.0 PDFs and a fit with the same dataset but with the new cut $Q_{\text {cut }}^{2}=3 \mathrm{GeV}^{2}$, denoted by NNPDF2.0RED (reduced). Also, in order to 

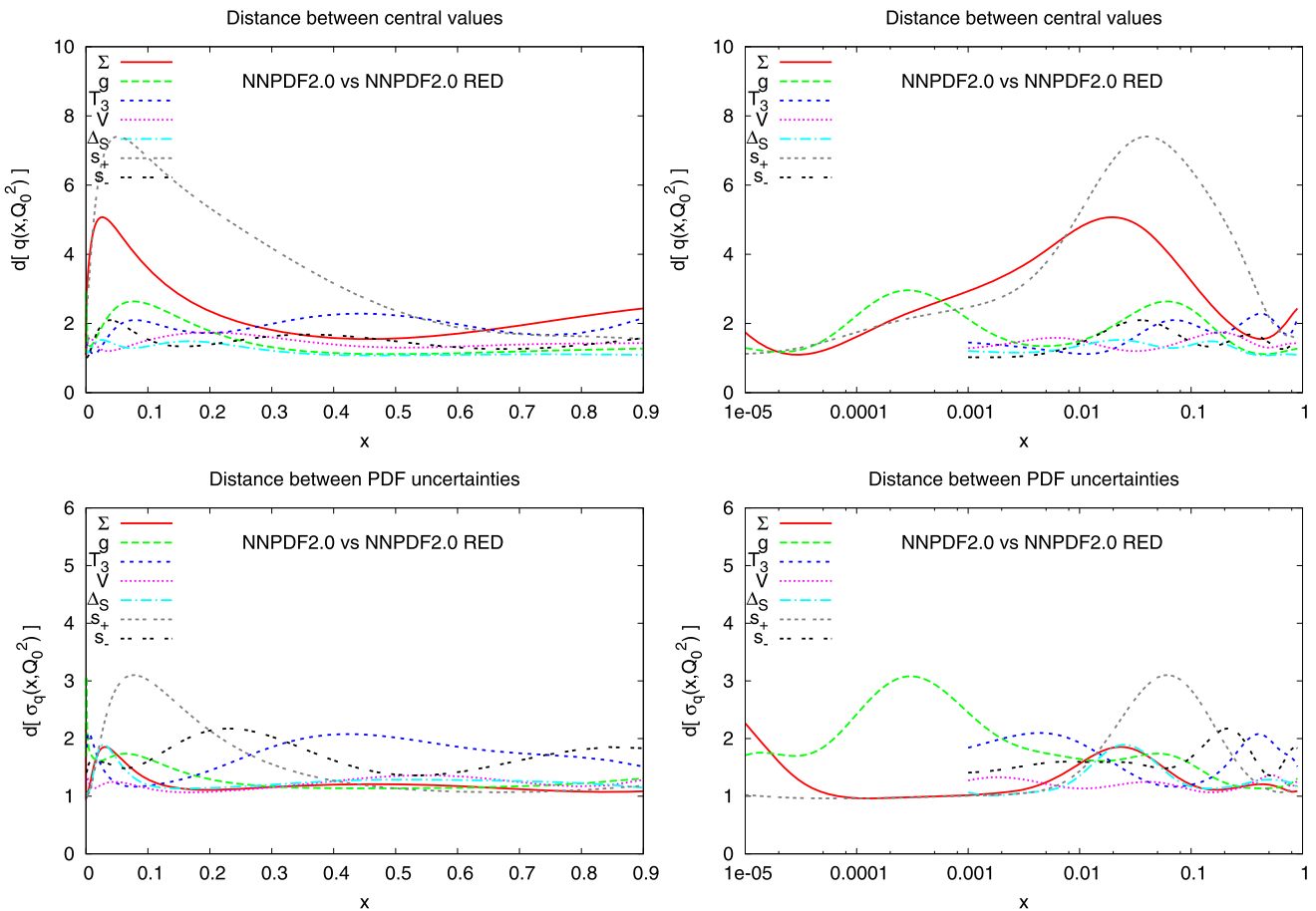

Fig. 18. Distance between the NNPDF2.0 PDF set and a fit to the same data but with $Q_{\text {cut }}^{2}=3 \mathrm{GeV}^{2}$ and the ZM-VFN scheme for all observables (NNPDF2.0 RED). All distances are computed from sets of $N_{\text {rep }}=100$ replicas.
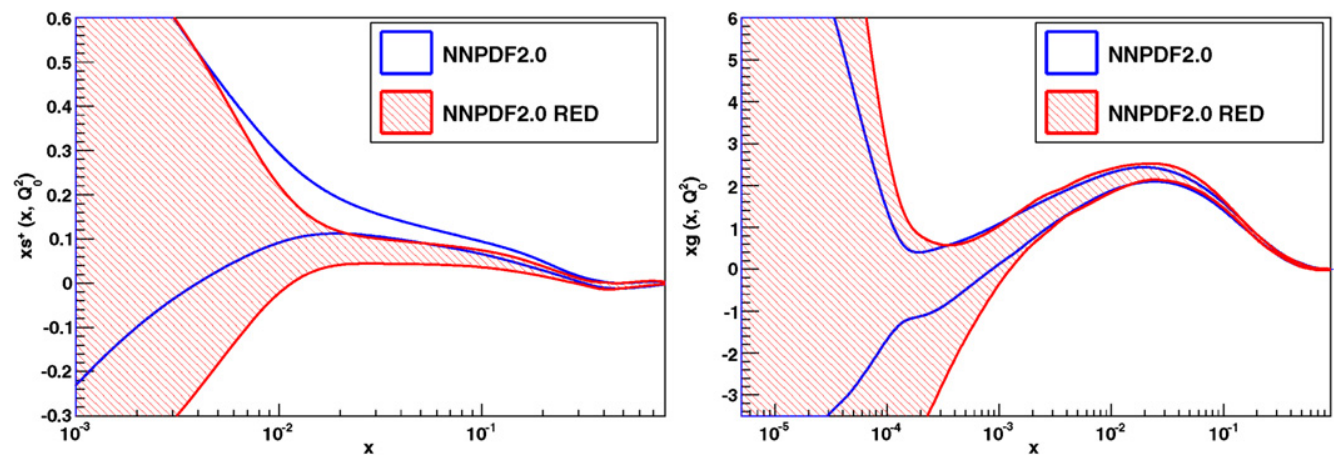

Fig. 19. Comparison of the small- $x$ total strangeness and gluon in NNPDF2.0 and in NNPDF2.0RED (the distances are shown in Fig. 18).

ease the subsequent discussion of the impact of heavy quark mass effects, in NNPDF2.0RED a pure ZM scheme is used for all observables, rather than the IZM scheme [55] used for dimuon data in Ref. [10].

The largest distances correspond to the medium- $x$ strange PDFs and the small $x$ gluon. These PDFs are shown in Fig. 19. The strange is rather smaller in the ZM as compared to the IZM scheme, where it was enhanced due to the approximate inclusion of charm suppression. The gluon is somewhat smaller at small- $x$ and with rather larger uncertainties, due 

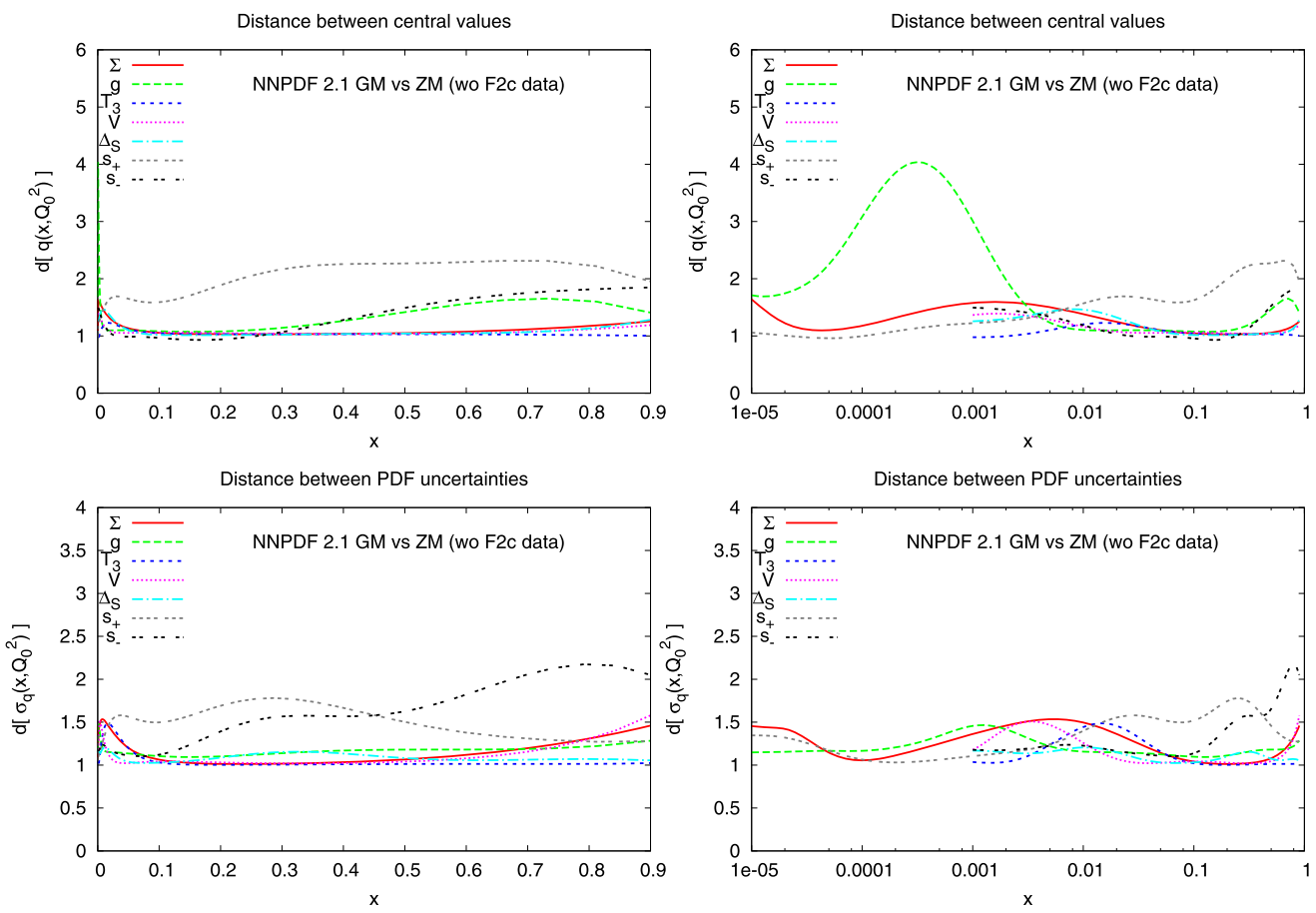

Fig. 20. Distance between the NNPDF2.1 PDF sets in the GM and in the ZM schemes, in both cases without HERA $F_{2}^{c}$ data. All distances are computed from sets of $N_{\text {rep }}=100$ replicas.
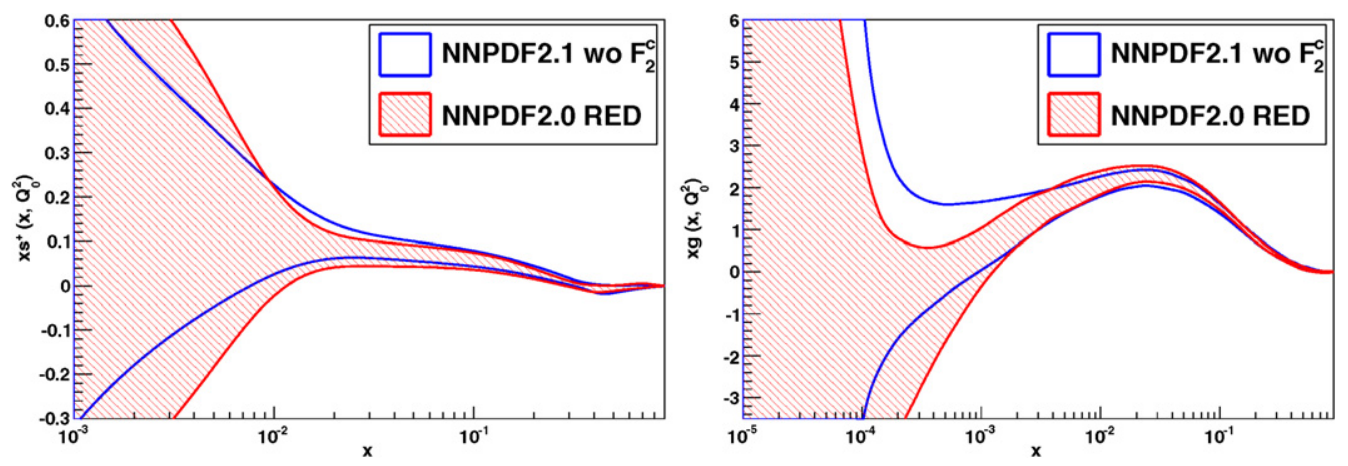

Fig. 21. Comparison of the small- $x$ total strangeness and gluon in NNPDF2.0RED and NNPDF2.1 without $F_{2}^{c}$ data (distances are shown in Fig. 20).

to the reduction in dataset at small- $x$ caused by the new kinematic cut. From the distances we see that the singlet is also modified, but one can check that this is completely due to the strange contribution to it.

- Impact of the general-mass scheme

The impact of the FONLL-A GM is assessed by now comparing the NNPDF2.0RED fit to an NNPDF2.1 fit without $F_{2}^{c}$ data: the dataset is identical, the only difference is in the treatment of heavy quark masses. The distances between these two sets are shown in Fig. 20. 

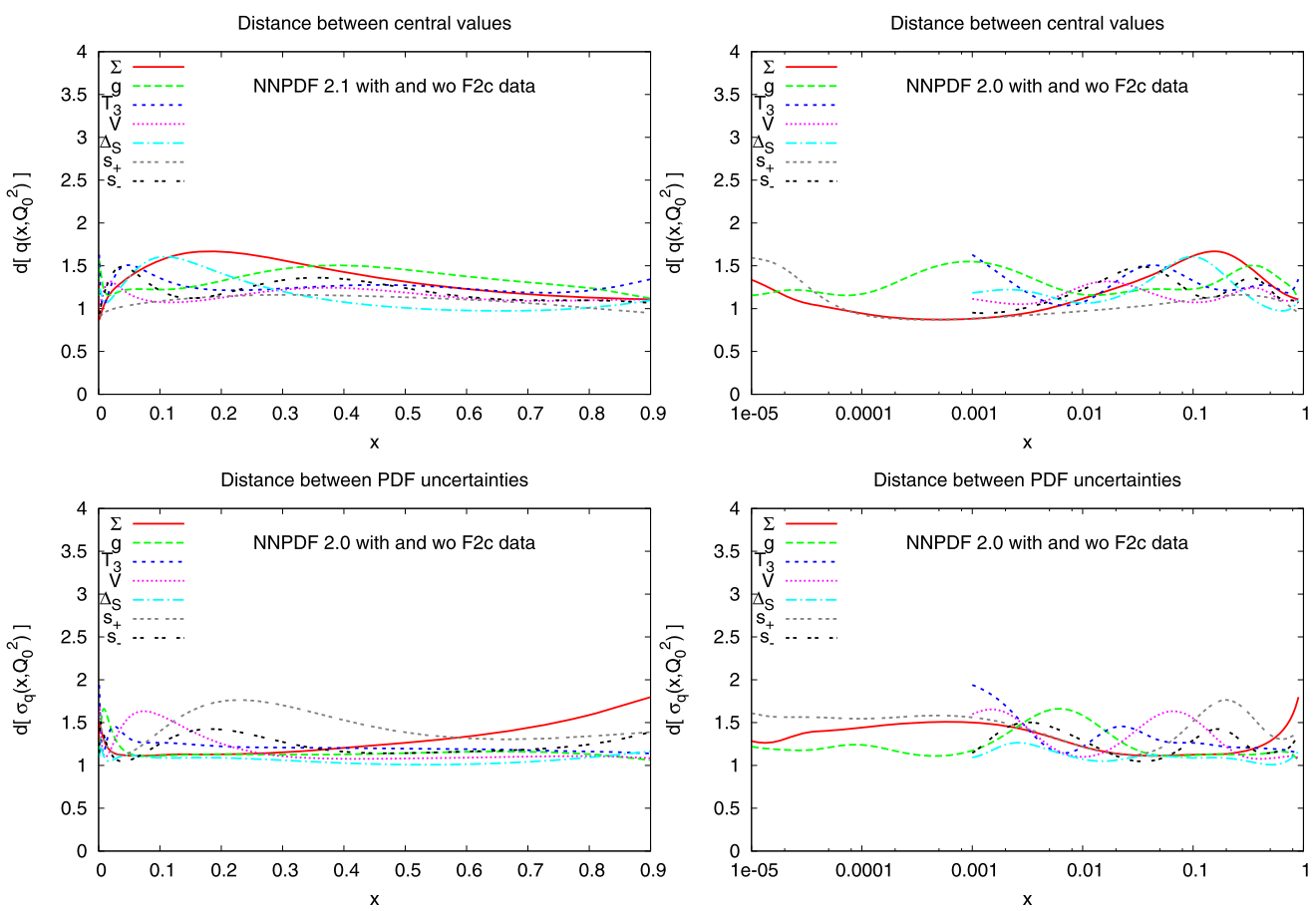

Fig. 22. Distance between NNPDF2.1 PDF sets with and without HERA $F_{2}^{c}\left(x, Q^{2}\right)$ data. Distances have been computed from sets of $N_{\text {rep }}=100$ replicas.

The impact of the inclusion of heavy quark masses is mostly on the small- $x$ gluon and, to a lesser extent, on medium- $x$ strangeness. These two PDFs are shown in Fig. 21. The GM scheme leads to a larger gluon for $x \lesssim 2 \cdot 10^{-3}$, as well as to a somewhat larger strangeness, but it leaves the singlet unaffected. This shows that indeed, as argued in Section 4.2, the relatively large total strangeness in NNPDF2.0 was due to the use of the IZM approximation for dimuon data, which overestimates charm mass effects.

- Impact of HERA $F_{2}^{c}$ data

The impact of HERA $F_{2}^{c}$ data is estimated comparing the results of the NNPDF2.1 fit with and without these data. The distances displayed in Fig. 22 show that current $F_{2}^{c}$ data have little effect: the two fits are almost statistically equivalent, with most distances of order one. This is partly due to the relatively large uncertainties on current these $F_{2}^{c}$, and also to the fact that low- $x$ and $Q^{2}$ data, which are most sensitive to the gluon PDF, are excluded by our kinematic cuts. Inclusion of $\mathcal{O}\left(\alpha_{s}^{2}\right)$ heavy quark mass effects (e.g. by means of the FONLL-B scheme) is necessary in order to take advantage of these data.

- Impact of threshold prescription in the GM scheme

Finally, we have repeated the NNPDF2.1 fit with a different treatment of subleading terms in the inclusion of heavy quark mass effects: namely, we have used FONLL-A but without the threshold damping factor in Eqs. (1), (32). Indeed, the benchmarking exercise of Ref. [19] suggests that the difference between these cases should provide a reasonable estimate of the spread of results obtained by including heavy quark masses according to different prescriptions. Distances are shown in Fig. 23: the PDFs that are most affected are the singlet and 

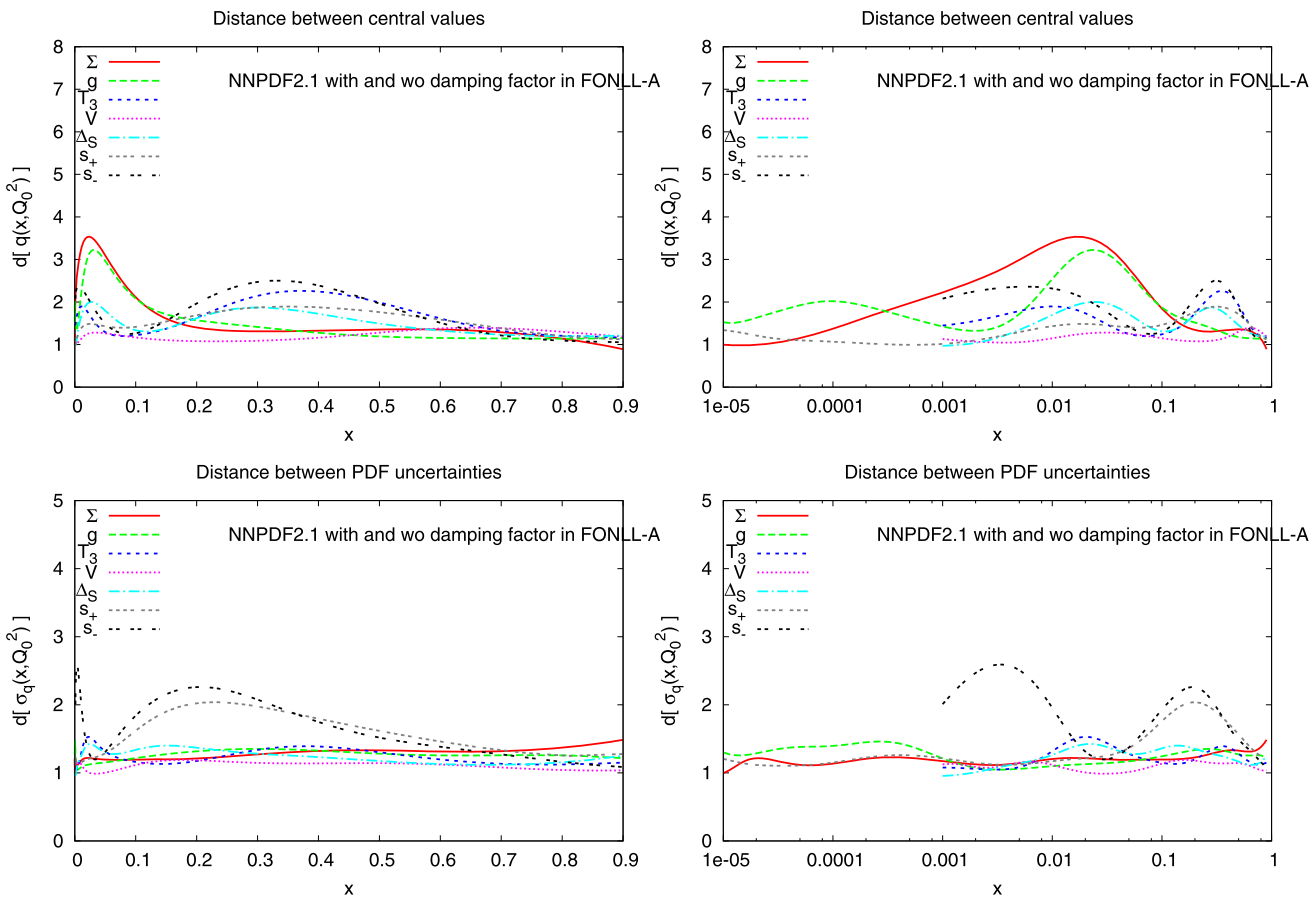

Fig. 23. Distance between the NNPDF2.1 reference set and the same set obtained without threshold damping factor in the computation of the FONLL-A structure functions.
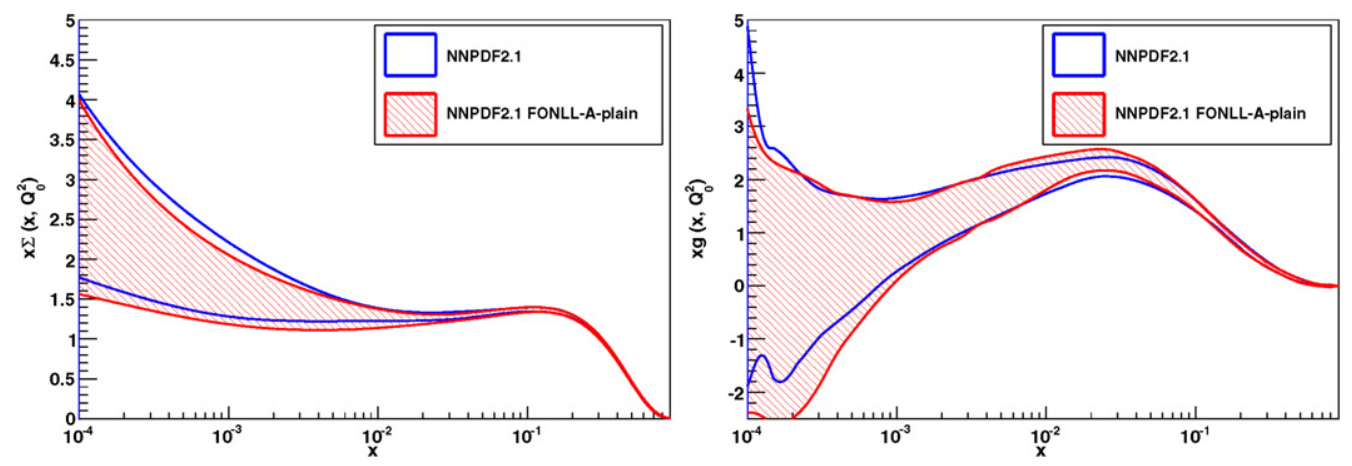

Fig. 24. The small- $x$ singlet and gluon PDFs, in the reference NNPDF2.1 set and in NNPDF2.1 obtained with FONLL-A without threshold damping factor (distances are shown in Fig. 23).

gluon PDFs at medium- $x$, shown in Fig. 24. Without damping factor, the $F_{2}^{c}$ structure function is closer to the massless result even at moderate $Q^{2}$, and this explains why the singlet PDF is somewhat smaller at medium- $x$. 
Table 6

Cross-sections for $W, Z, t \bar{t}$ and Higgs production at the LHC at $\sqrt{s}=7 \mathrm{TeV}$ and the associated PDF uncertainties. All quantities have been computed at NLO using MCFM for the NNPDF2.1, NNPDF2.0, CT10 and MSTW08 PDF sets. All uncertainties shown are one- $\sigma$.

\begin{tabular}{|c|c|c|c|}
\hline & $\sigma\left(W^{+}\right) B_{l v}[\mathrm{nb}]$ & $\sigma\left(W^{-}\right) B_{l v}[\mathrm{nb}]$ & $\sigma\left(Z^{0}\right) B_{l l}[\mathrm{nb}]$ \\
\hline \multirow{2}{*}{$\begin{array}{l}\text { NNPDF2.0 } \\
\text { NNPDF2.1 }\end{array}$} & $5.84 \pm 0.14$ & $3.97 \pm 0.09$ & $0.91 \pm 0.02$ \\
\hline & $5.99 \pm 0.14$ & $4.09 \pm 0.09$ & $0.93 \pm 0.02$ \\
\hline \multirow{2}{*}{$\begin{array}{l}\mathrm{CT} 10-\alpha_{s}=0.118 \\
\mathrm{CT} 10-\alpha_{s}=0.119\end{array}$} & $6.00 \pm 0.13$ & $4.10 \pm 0.09$ & $0.94 \pm 0.02$ \\
\hline & $6.04 \pm 0.13$ & $4.13 \pm 0.09$ & $0.95 \pm 0.02$ \\
\hline \multirow{3}{*}{$\begin{array}{l}\text { MSTW08- } \alpha_{s}=0.119 \\
\text { MSTW08- } \alpha_{s}=0.120\end{array}$} & $5.91 \pm 0.11$ & $4.16 \pm 0.08$ & $0.94 \pm 0.02$ \\
\hline & $5.95 \pm 0.11$ & $4.19 \pm 0.08$ & $0.95 \pm 0.02$ \\
\hline & $\sigma(t \bar{t})[\mathrm{pb}]$ & $\sigma\left(H, m_{H}=120 \mathrm{GeV}\right)[\mathrm{pb}]$ & \\
\hline NNPDF2.0 & $168 \pm 7$ & $11.59 \pm 0.22$ & \\
\hline NNPDF2.1 & $170 \pm 5$ & $11.64 \pm 0.17$ & \\
\hline $\mathrm{CT} 10-\alpha_{s}=0.118$ & $158 \pm 7$ & $10.99 \pm 0.21$ & \\
\hline $\mathrm{CT} 10-\alpha_{s}=0.119$ & $161 \pm 7$ & $11.17 \pm 0.21$ & \\
\hline MSTW08 $-\alpha_{S}=0.119$ & $164 \pm 5$ & $11.48 \pm 0.18$ & \\
\hline MSTW08 $-\alpha_{s}=0.120$ & $168 \pm 5$ & $11.69 \pm 0.18$ & \\
\hline
\end{tabular}

\section{Phenomenological implications}

In this section we discuss the implications of the NNPDF2.1 set for LHC physics. We begin comparing the prediction for LHC benchmark cross-sections obtained using NNPDF2.1 and NNPDF2.0. This comparison allows us to assess the impact of heavy quark mass effects. We also compare to predictions obtained using the CT10 and MSTW08 sets, both using their preferred values of $\alpha_{s}$ and with a common value. We then compare parton luminosities relevant for LHC processes and determine correlations between PDFs and some observables. Next, we determine the correlation between PDFs and $\alpha_{s}$ and discuss PDF sets with varying $\alpha_{s}$, which are needed to compute the combined PDF $+\alpha_{s}$ uncertainties, and present sets in which PDF and $\alpha_{s}$ uncertainties are pre-combined. Finally, we briefly revisit implications of the strangeness asymmetry on the $\mathrm{NuTeV}$ anomaly, confirming our previous result that the anomaly disappears once the strangeness asymmetry is properly determined. We conclude with a comparison of NNPDF2.1 results with published HERA $F_{L}$ and $F_{2}^{c}$ data and predictions for the upcoming $F_{L}$ and $F_{2}^{c}$ HERA combined datasets.

\subsection{LHC benchmark cross-sections}

The assessment of the theoretical uncertainties on LHC standard candles is especially important now that the first $7 \mathrm{TeV}$ LHC results on inclusive cross-sections are appearing [70-73]. In this section we present results at $\sqrt{s}=7 \mathrm{TeV}$ and $\sqrt{s}=14 \mathrm{TeV}$ for $W^{ \pm}, Z^{0}, t \bar{t}$ and Higgs production via gluon fusion with $m_{H}=120 \mathrm{GeV}$. All observables are computed at NLO QCD using MCFM [74,75].

In Tables 6 and 7, and the corresponding Figs. 25 and 27, we compare the predictions for these cross-sections obtained using the NNPDF2.1, NNPDF2.0, CT10 and MSTW08 sets. In the case of the last two sets, we show results both using the respective default value of $\alpha_{S}\left(M_{Z}\right)$ and at the common value of $\alpha_{s}\left(M_{Z}\right)=0.119$, obtained using the PDF sets of Refs. [76,77]. 

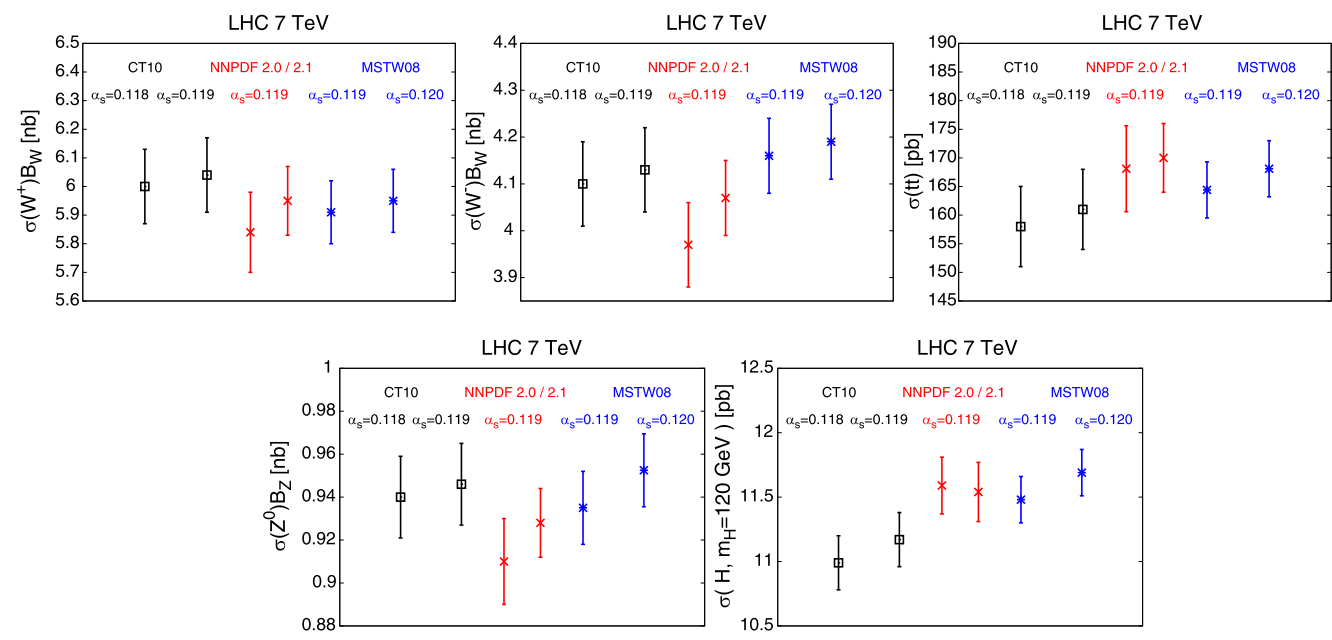

Fig. 25. Graphical representation of the results of Table 6.

Predictions obtained using the NNPDF2.0 and NNPDF2.1 PDF sets mostly differ because of heavy quark mass effects, but other differences such as different kinematic cuts also play a role. As can be seen from Fig. 25 and Table 6, the differences between NNPDF2.0 and NNPDF2.1 are at most at the one- $\sigma$ level for $W^{ \pm}$and $Z$ production $7 \mathrm{TeV}$, while predictions for the $t \bar{t}$ and Higgs are essentially unchanged: these observables are only minimally affected by the heavy quark treatment.

NNPDF2.1 predictions are in rather good agreement with MSTW08 for all observables, though differences with CT10 are somewhat larger, especially for observables which are most sensitive to the gluon distribution, like Higgs and $t \bar{t}$ production. The use of a common value for the strong coupling $\alpha_{s}$ leads to better agreement between predictions, especially for processes which depend on $\alpha_{s}$ already at leading order such as Higgs production in gluon fusion [21]. In Fig. 26 first measurements by the ATLAS and CMS experiments [70,71] are compared to these NLO predictions: with their large uncertainties, dominated by the current large $(\mathcal{O}(11 \%))$ luminosity uncertainty, they cannot yet provide constraint on PDFs (of course, NNLO corrections, which are at the few percent level, are irrelevant on the scale of these uncertainties).

At $\sqrt{s}=14 \mathrm{TeV}$ we expect the effect of the heavy quark treatment to be larger: results are collected in Fig. 27 and Table 7. In this case, the upwards shift in the $W^{ \pm}$and $Z$ cross-sections from NNPDF2.0 to NNPDF2.1 is at or just above the one- $\sigma$ level, while as before Higgs and top-pair cross-sections are essentially unchanged. The comparison with CT10 and MSTW08 is similar as before, but with the agreement somewhat better for the Higgs and somewhat worse for top.

Related important observables at the LHC are the $W^{+} / W^{-}$and $W / Z$ cross-section ratios. These have generally reduced experimental uncertainties, since e.g. normalization uncertainties cancel in the ratio. Predictions at 7 and 14 TeV for NNPDF2.1, CT10 and MSTW08 are compared in Fig. 28. For these observables the dependence on $\alpha_{s}$ is negligible. The agreement for crosssection ratios seems to be worse than for total cross-sections: for example for the $W^{+} / W^{-}$ratio CT10 and NNPDF2.1 are in good agreement but MSTW08 is lower by more than two- $\sigma$. The agreement for the $W / Z$ ratio is better but still marginal at $7 \mathrm{TeV}$. 


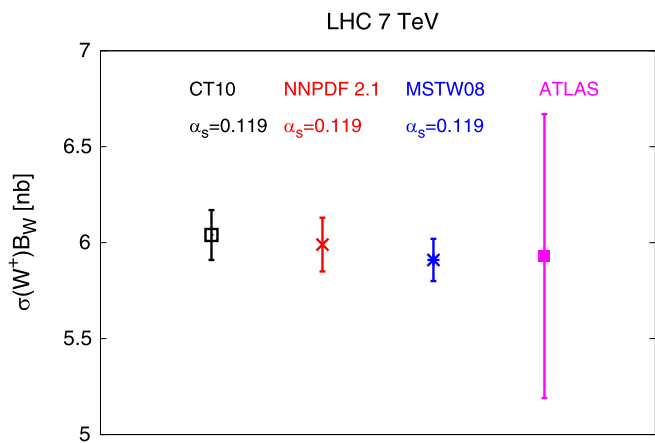

LHC $7 \mathrm{TeV}$

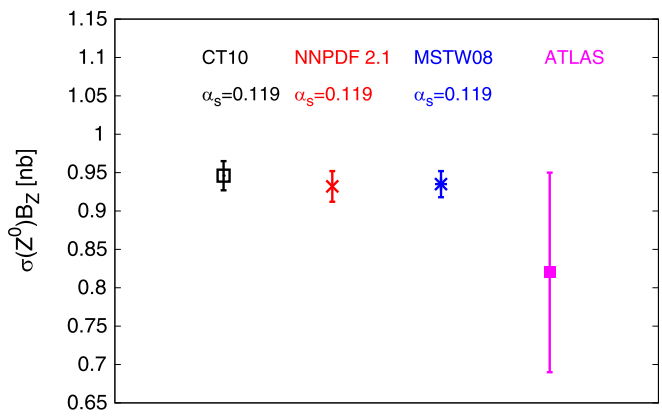

LHC 7 TeV

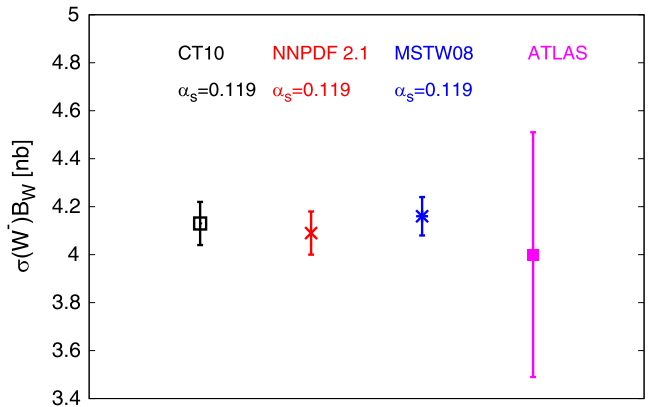

LHC $7 \mathrm{TeV}$

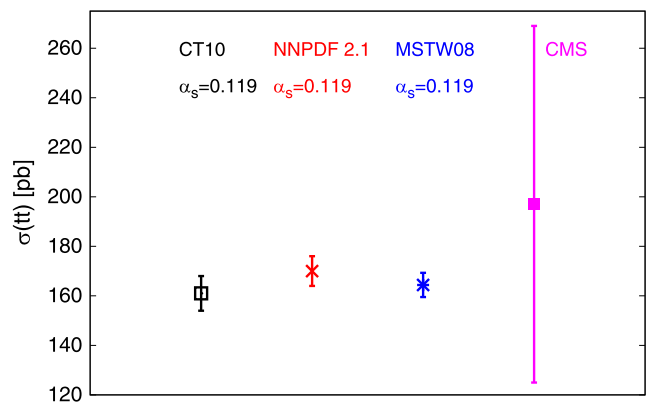

Fig. 26. LHC measurements of the $W^{ \pm}, Z$ and $t \bar{t}$ cross-sections at $\sqrt{s}=7$ TeV from the ATLAS [70] and CMS experiments [71] compared to the predictions of Fig. 25.
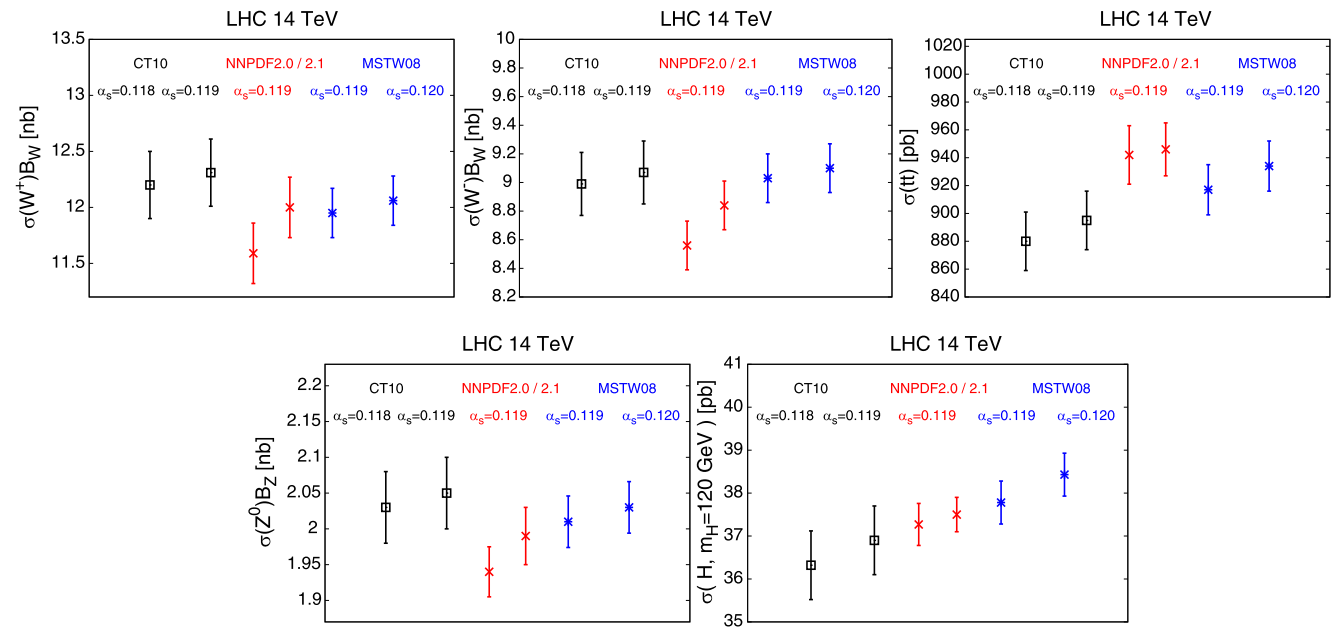

Fig. 27. Graphical representation of the results of Table 7.

The correlation between PDFs and physical observables quantifies the relevance of each PDF for different observables (and conversely) as a function of $x[4,15]$. As an illustration with NNPDF2.1, we have computed the correlations between PDFs and vector boson production total 
Table 7

Same as Table 6 for the LHC at $\sqrt{s}=14 \mathrm{TeV}$.

\begin{tabular}{llll}
\hline & $\sigma\left(W^{+}\right) B_{l v}[\mathrm{nb}]$ & $\sigma\left(W^{-}\right) B_{l v}[\mathrm{nb}]$ & $\sigma\left(Z^{0}\right) B_{l l}[\mathrm{nb}]$ \\
\hline NNPDF2.0 & $11.59 \pm 0.27$ & $8.56 \pm 0.17$ & $1.94 \pm 0.04$ \\
NNPDF2.1 & $12.00 \pm 0.27$ & $8.84 \pm 0.17$ & $1.99 \pm 0.04$ \\
CT10 $-\alpha_{s}=0.118$ & $12.20 \pm 0.30$ & $9.00 \pm 0.22$ & $2.03 \pm 0.05$ \\
CT10 $-\alpha_{s}=0.119$ & $12.31 \pm 0.30$ & $9.07 \pm 0.22$ & $2.05 \pm 0.05$ \\
MSTW08 $-\alpha_{s}=0.119$ & $11.95 \pm 0.22$ & $9.03 \pm 0.17$ & $2.01 \pm 0.04$ \\
MSTW08 $-\alpha_{s}=0.120$ & $12.06 \pm 0.22$ & $9.10 \pm 0.17$ & $2.03 \pm 0.04$ \\
\hline
\end{tabular}

\begin{tabular}{lll}
\hline & $\sigma(t \bar{t})[\mathrm{pb}]$ & $\sigma\left(H, m_{H}=120 \mathrm{GeV}\right)[\mathrm{pb}]$ \\
\hline NNPDF2.0 & $942 \pm 21$ & $37.3 \pm 0.50$ \\
NNPDF2.1 & $946 \pm 19$ & $37.5 \pm 0.40$ \\
CT10 $-\alpha_{s}=0.118$ & $880 \pm 21$ & $36.32 \pm 0.80$ \\
CT10 $-\alpha_{s}=0.119$ & $895 \pm 21$ & $36.90 \pm 0.80$ \\
MSTW08 $-\alpha_{s}=0.119$ & $917 \pm 18$ & $37.78 \pm 0.50$ \\
MSTW08 $-\alpha_{s}=0.120$ & $934 \pm 18$ & $38.43 \pm 0.50$ \\
\hline
\end{tabular}
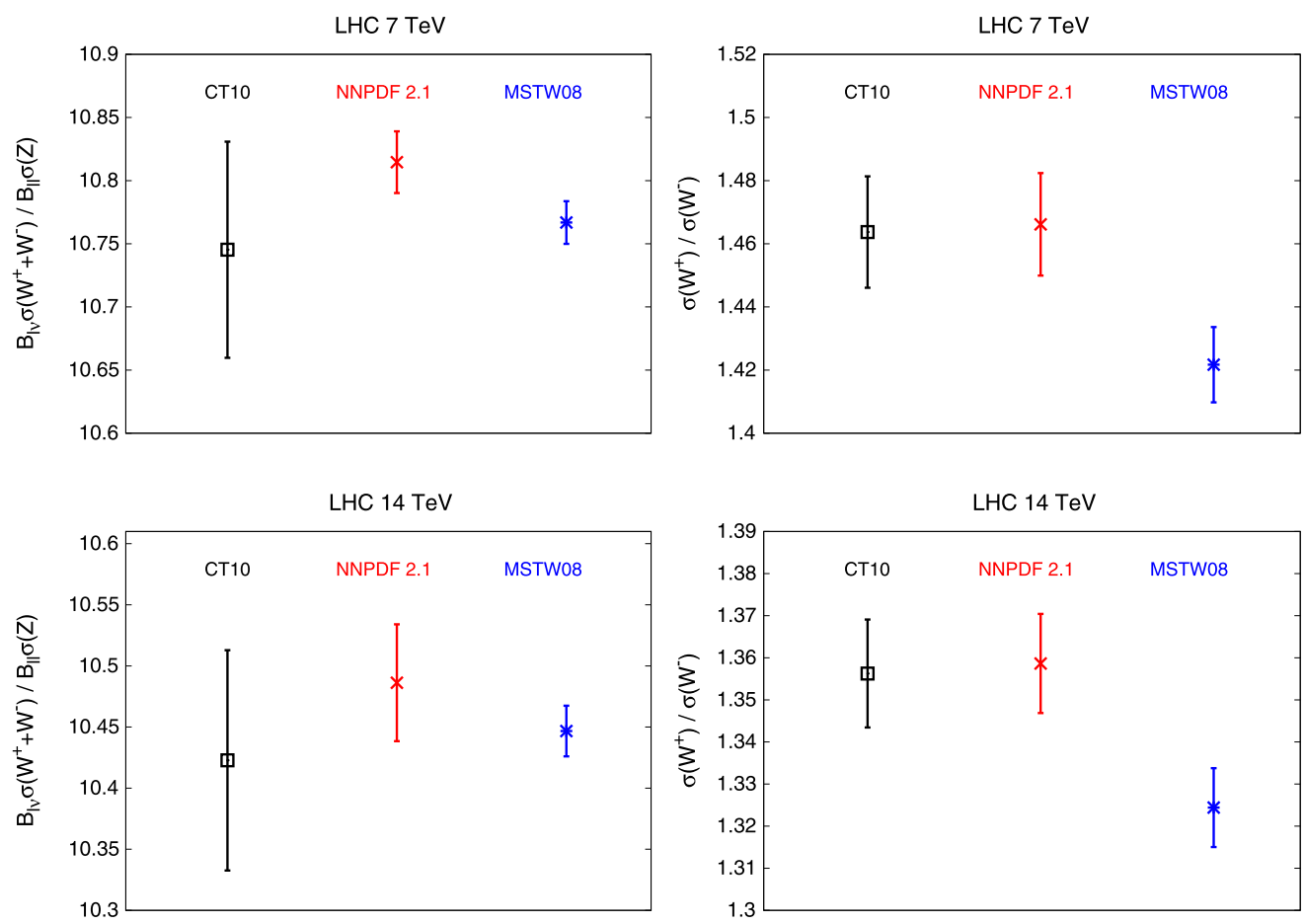

Fig. 28. Comparison between predictions from different PDF sets for the $W^{+} / W^{-}$and $W / Z$ ratios at the LHC $\sqrt{s}=$ $7 \mathrm{TeV}$ (upper plots) and $\sqrt{s}=14 \mathrm{TeV}$ (lower plots). 


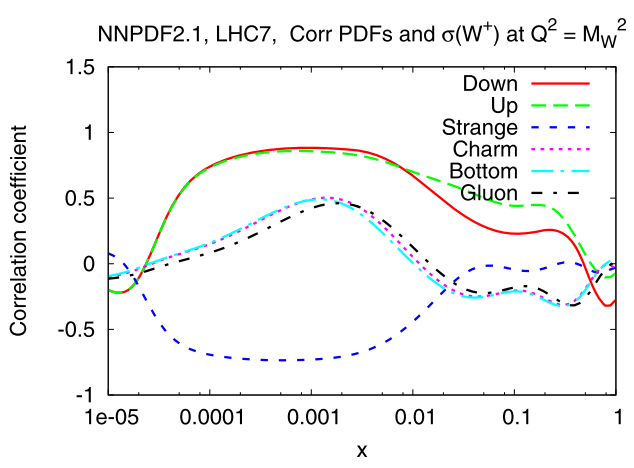

NNPDF2.1, LHC7, Corr PDFs and $\sigma\left(\mathrm{W}^{+}\right) / \sigma\left(\mathrm{W}^{-}\right)$at $\mathrm{Q}^{2}=\mathrm{M}_{\mathrm{W}}{ }^{2}$

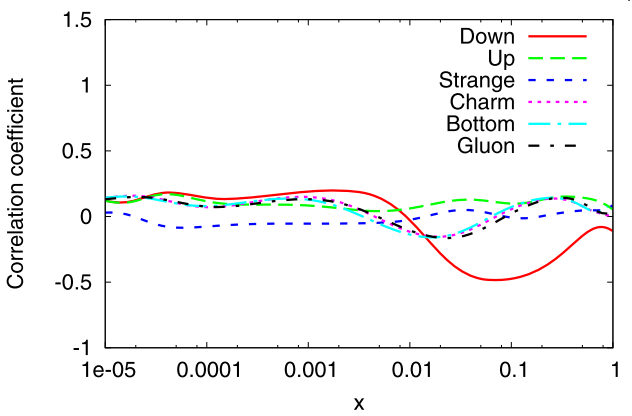

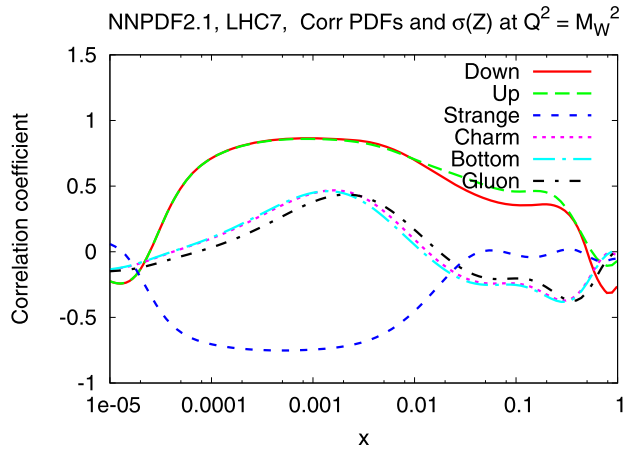

NNPDF2.1, LHC7, Corr PDFs and $\sigma(\mathrm{W}) / \sigma(\mathrm{Z})$ at $\mathrm{Q}^{2}=\mathrm{M}_{\mathrm{W}}{ }^{2}$

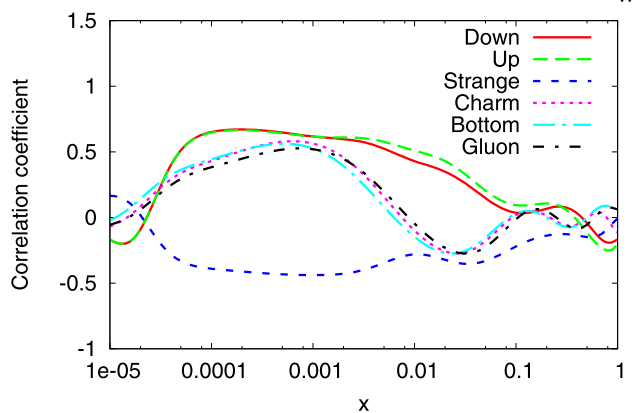

Fig. 29. The correlation between PDFs and vector boson production total cross-sections (upper plots) and their ratios (lower plots) for LHC $7 \mathrm{TeV}$. Correlations for $W^{-}$(not shown) are very similar.

cross-sections and their ratios at LHC 7 TeV. Results are shown in Fig. 29. The total $W$ and $Z$ cross-sections are as expected mostly correlated with the $u$ and $d$ sea quarks and anticorrelated with the strange quarks, the correlation with the gluon (and the heavy flavors generated dynamically from it) being milder. It is also interesting to note that correlations between PDFs and the corresponding physical observable are only moderately reduced in the $W / Z$ ratio as compared to the individual cross-sections, and they are instead almost suppressed in the $W^{+} / W^{-}$ratio. This observation suggests that the latter ratio should be less sensitive to PDF uncertainties.

As for NNPDF2.0 [10], we have produced variants of the NNPDF2.1 fit based on reduced datasets: DIS only, DIS and inclusive jet data only. These fits are useful to study the impact on PDF of the various observables used in the global fit. Results for LHC cross-sections at $7 \mathrm{TeV}$ determined using these fits are collected in Table 8. For these very inclusive observables, it turns out that a purely DIS fit already provides a rather good approximation, though this need not be always the case for other observables.

The inclusion of heavy quark mass effects has a theoretical ambiguity due to subleading terms. A full study of theoretical uncertainties on PDFs has never been performed and goes beyond the scope of this paper. However, we provide here a first estimate of the uncertainty related to the inclusion of heavy quark mass effects to $\mathcal{O}\left(\alpha_{s}\right)$ by comparing results obtained from the three sets discussed in Section 4.3: NNPDF2.0 RED (without heavy quark mass terms, but the same kinematic cuts NNPDF2.1), the default NNPDF2.1, and NNPDF2.1 without damping terms in the FONLL-A method. Results are shown in Table 9 and in Fig. 30. As expected, results obtained without damping prescription sit half way between NNPDF2.0RED and NNPDF2.1 at $7 \mathrm{TeV}$, and 
Table 8

Cross-sections for $W, Z, t \bar{t}$ and Higgs production at the LHC at $\sqrt{s}=7 \mathrm{TeV}$ and the associated PDF uncertainties for the reference NNPDF2.1 set compared to those obtained using sets determined from reduced datasets: DIS only, DIS + JET.

\begin{tabular}{llll}
\hline & $\sigma\left(W^{+}\right) B_{l v}[\mathrm{nb}]$ & $\sigma\left(W^{-}\right) B_{l v}[\mathrm{nb}]$ & $\sigma\left(Z^{0}\right) B_{l l}[\mathrm{nb}]$ \\
\hline NNPDF2.1 DIS & $6.03 \pm 0.11$ & $4.15 \pm 0.08$ & $0.940 \pm 0.014$ \\
NNPDF2.1 DIS + JET & $6.03 \pm 0.12$ & $4.14 \pm 0.08$ & $0.939 \pm 0.015$ \\
NNPDF2.1 & $5.99 \pm 0.14$ & $4.09 \pm 0.09$ & $0.932 \pm 0.020$ \\
\hline
\end{tabular}

\begin{tabular}{lll}
\hline & $\sigma(t \bar{t})[\mathrm{pb}]$ & $\sigma\left(H, m_{H}=120 \mathrm{GeV}\right)[\mathrm{pb}]$ \\
\hline NNPDF2.1 DIS & $167 \pm 7$ & $11.66 \pm 0.21$ \\
NNPDF2.1 DIS + JET & $170 \pm 5$ & $11.66 \pm 0.22$ \\
NNPDF2.1 & $170 \pm 5$ & $11.64 \pm 0.17$ \\
\hline
\end{tabular}

Table 9

Cross-sections for $W, Z, t \bar{t}$ and Higgs production at the LHC at $\sqrt{s}=7 \mathrm{TeV}$ and the associated PDF uncertainties for the reference NNPDF2.1 set compared to those obtained using sets with different treatment of heavy quarks: NNPDF2.0RED, without heavy quark mass effects, and NNPDF2.1 FONLL-A plain with heavy quark mass effects but without threshold damping terms.

\begin{tabular}{llll}
\hline $7 \mathrm{TeV}$ & $\sigma\left(W^{+}\right) B_{l v}[\mathrm{nb}]$ & $\sigma\left(W^{-}\right) B_{l \nu}[\mathrm{nb}]$ & $\sigma\left(Z^{0}\right) B_{l l}[\mathrm{nb}]$ \\
\hline NNPDF2.1 & $5.99 \pm 0.14$ & $4.09 \pm 0.09$ & $0.93 \pm 0.02$ \\
NNPDF2.0 RED & $5.81 \pm 0.13$ & $3.98 \pm 0.08$ & $0.91 \pm 0.02$ \\
NNPDF2.1 FONLL-A plain & $5.90 \pm 0.12$ & $4.03 \pm 0.08$ & $0.92 \pm 0.02$ \\
\hline $14 \mathrm{TeV}$ & $\sigma\left(W^{+}\right) B_{l v}[\mathrm{nb}]$ & $\sigma\left(W^{-}\right) B_{l v}[\mathrm{nb}]$ & $\sigma\left(Z^{0}\right) B_{l l}[\mathrm{nb}]$ \\
\hline NNPDF2.1 & $12.00 \pm 0.27$ & $8.84 \pm 0.17$ & $1.99 \pm 0.04$ \\
NNPDF2.0 RED & $11.57 \pm 0.25$ & $8.57 \pm 0.17$ & $1.93 \pm 0.04$ \\
NNPDF2.1 FONLL-A plain & $11.82 \pm 0.22$ & $8.72 \pm 0.15$ & $1.96 \pm 0.03$ \\
\hline
\end{tabular}

closer to the latter at $14 \mathrm{TeV}$. As discussed in Section 4.3, the difference between the NNPDF2.1 results with and without damping terms can be taken as a conservative estimate of the theoretical uncertainty associated to the uncertainty in the inclusion of heavy quark mass effects to $\mathcal{O}\left(\alpha_{s}\right)$.

\subsection{Parton luminosities}

The processes discussed in Section 5.1 are a small subset of the LHC observables which are sensitive to PDFs. A detailed, systematic study of these would be quite interesting; however, a good deal of information can be gathered by simply studying parton luminosities. Following Ref. [78], we define the parton luminosity

$$
\Phi_{i j}\left(M_{X}^{2}\right)=\frac{1}{s} \int_{\tau}^{1} \frac{d x_{1}}{x_{1}} f_{i}\left(x_{1}, M_{X}^{2}\right) f_{j}\left(\tau / x_{1}, M_{X}^{2}\right),
$$

where $f_{i}\left(x, M^{2}\right)$ is a PDF and $\tau \equiv M_{X}^{2} / s$. We consider in particular the gluon-gluon luminosity, the various heavy quark-antiquark luminosity, and the quark-gluon and quark-quark luminosity respectively defined as

$$
\Phi_{q g} \equiv \sum_{i=1}^{N_{f}} \Phi_{q_{i} g} ; \quad \Phi_{q q} \equiv \sum_{i=1}^{N_{f}} \Phi_{q_{i} \bar{q}_{i}} .
$$



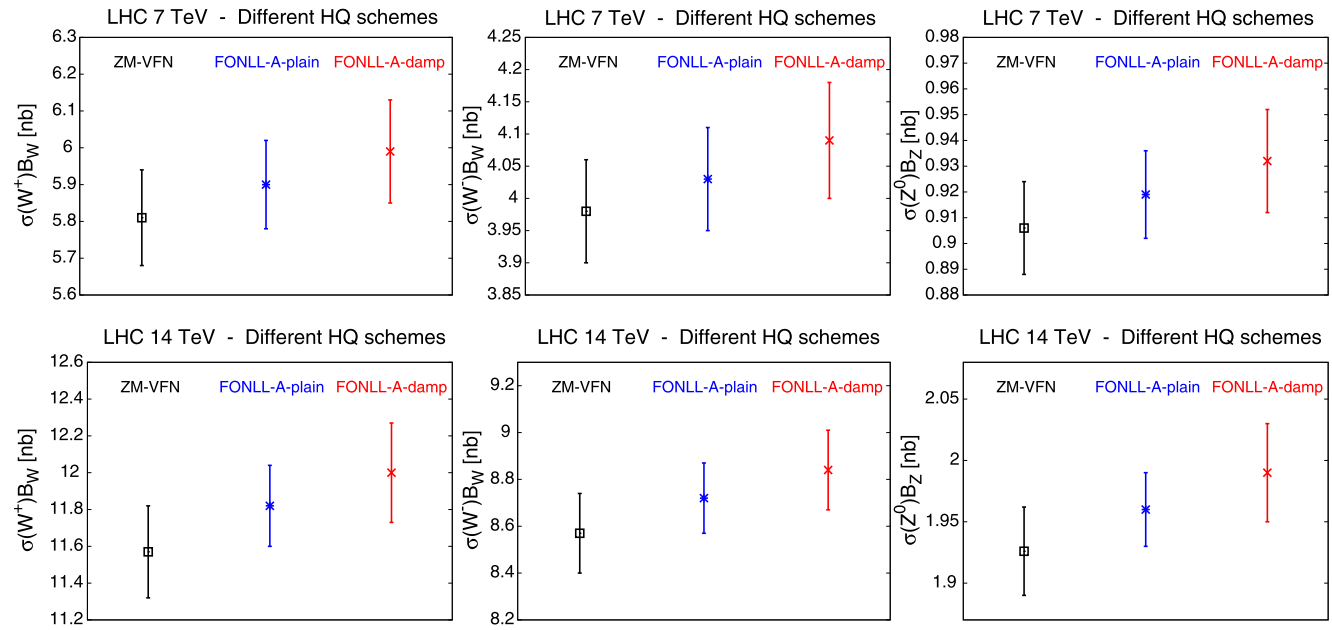

Fig. 30. Graphical representation of the results of Table 9.
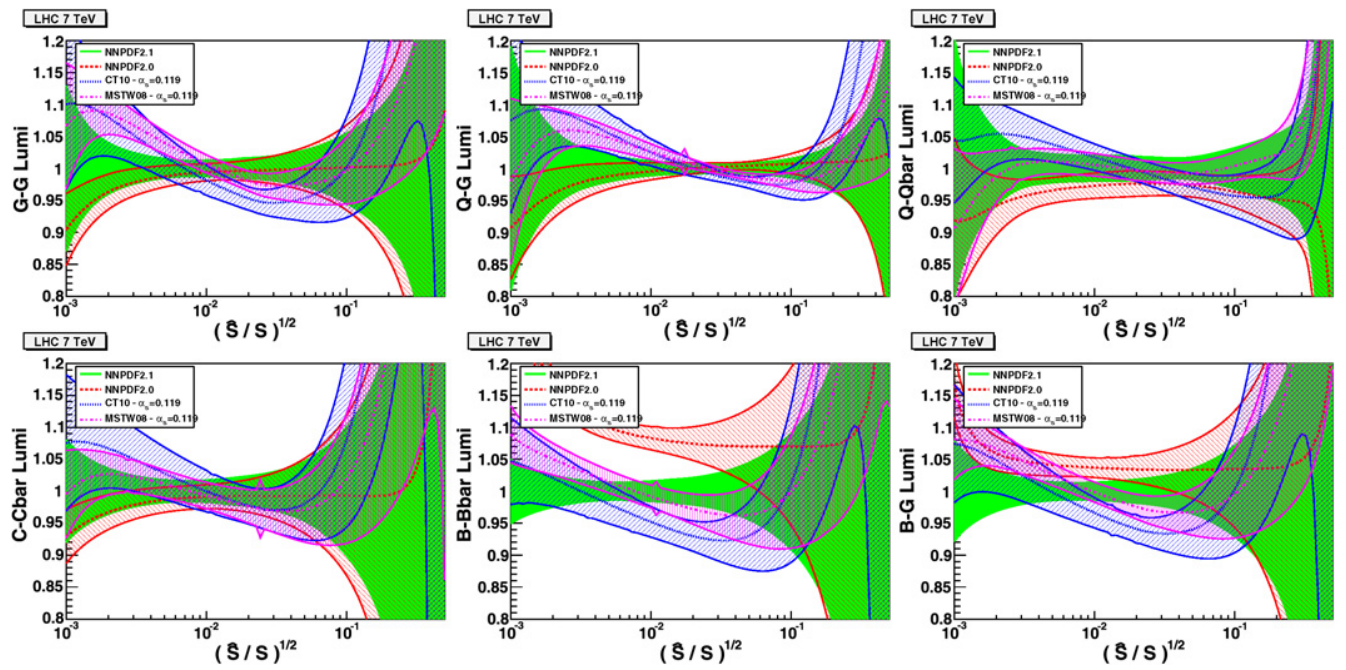

Fig. 31. The parton luminosities (34)-(35) for NNPDF2.1 compared to NNPDF2.0, CT10 and MSTW2008: from left to right and from top to bottom $\Phi_{g g}, \Phi_{q g}, \Phi_{q q}, \Phi_{c c}, \Phi_{b b}, \Phi_{b g}$. All luminosities are plotted as ratios to the NNPDF2.1 central value. PDF sets with $\alpha_{S}\left(M_{Z}\right)=0.119$ have been used in all cases. All uncertainties shown are one- $\sigma$.

Parton luminosities for the NNPDF2.1, NNPDF2.0, CT10 and MSTW08 sets, normalized to the NNPDF2.1 central prediction, are shown in Fig. 31 for $\mathrm{LHC} \sqrt{s}=7 \mathrm{TeV}$, all determined with $\alpha_{s}\left(M_{Z}\right)=0.119$. In Fig. 32 we also compare directly the relative uncertainties on the luminosity for each set. These comparisons show good agreement between global fits at the one- $\sigma$ level, although in some cases, such as the gluon-gluon luminosity at intermediate invariant masses, the agreement is only marginal. Uncertainties blow up both at very small and large values of $M_{X}$ for all sets. Differences between different sets are larger in these regions: for example NNPDF2.1 uncertainties at small $M_{X}$ are rather larger, for luminosities that involve the gluon PDF. The peculiar behavior of the bottom luminosity for NNPDF2.0 is due to the fact that in this set 

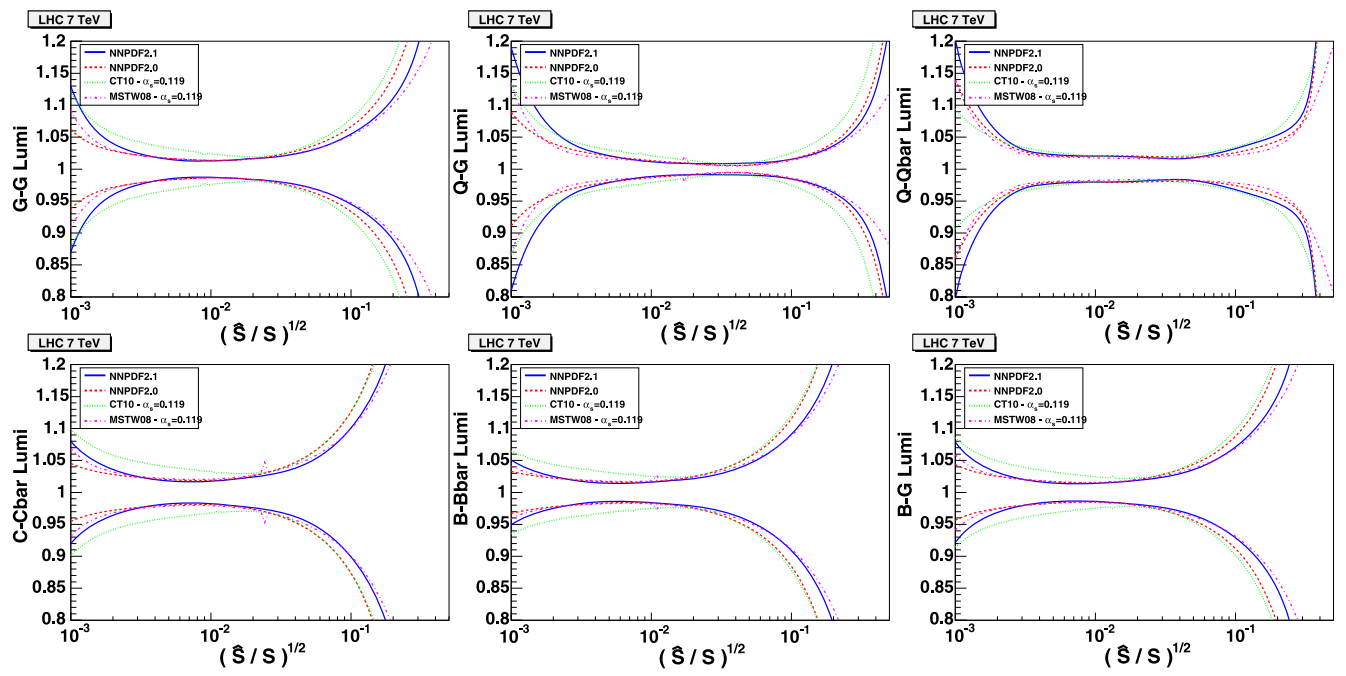

Fig. 32. Same as Fig. 31, but will all luminosities normalized to their respective central values.

$m_{b}=4.3 \mathrm{GeV}$, while $m_{b}=4.75 \mathrm{GeV}$ for all other sets. The dependence of results on the values of the heavy quark masses will be discussed in Section 6 below.

\subsection{The value of $\alpha_{S}\left(M_{Z}\right)$}

We now consider the correlation between NNPDF2.1 partons and the value of $\alpha_{s}$. To this purpose, we provide sets with $\alpha_{s}\left(M_{Z}\right)$ in the range from 0.114 to 0.124 in steps of 0.001 . PDFs from fits performed using different values of $\alpha_{s}$ are show in Fig. 33. Results are similar to those obtained with NNPDF2.0 [20]: as expected, the most sensitive PDF is the gluon. To quantify this it is useful to compute the correlation between PDFs and $\alpha_{s}$ (as defined in Eq. (82) of Ref. [20]). We determine it assuming the uncertainty on $\alpha_{s}$ to be $\delta \alpha_{s}=0.0012$ at the $68 \%$ C.L. Results are plotted in Fig. 34 as a function of $x$, both at $Q^{2}=2 \mathrm{GeV}^{2}$ and $Q^{2}=10^{4} \mathrm{GeV}^{2}$. Clearly, because of asymptotic freedom, correlations are weaker at high scale.

Following the procedure outlined in Ref. [21], it is possible to combine sets with different values of $\alpha_{s}$ to compute the combined PDF $+\alpha_{s}$ uncertainty on any given observable. This procedure has the advantage that both the central value and the uncertainty on the strong coupling are not fixed a priori but can be chosen by the PDF user. In order to simplify this procedure, we provide prepacked PDF sets with combined PDF $+\alpha_{s}$ uncertainty. Once a central value and uncertainty for $\alpha_{S}\left(M_{Z}\right)$ are assumed, this is done by constructing a set of $N_{\text {rep }}$ replicas, extracted from the original sets with different $\alpha_{s}$, in such a way that the prepacked sets contains a number of replicas for each value of $\alpha_{s}$ which corresponds to a Gaussian distribution with given mean and standard deviation [21] (of course, any other distribution could be used). The statistical accuracy of the prediction obtained using the prepacked sets scales with the number of replicas $N_{\text {rep }}$.

We have produced prepacked PDF $+\alpha_{s}$ uncertainty sets with $\alpha_{s}\left(M_{z}\right)=0.119$ and uncertainties $\delta_{\alpha_{s}}=0.0012$ and $\delta_{\alpha_{s}}=0.002$ as one- $\sigma$ errors. These values have been chosen to agree with the PDF4LHC recommendation [79] for the combination of PDF $+\alpha_{s}$ uncertainties. Sets with any other values are easily produced and are available upon request. For completeness, we have produced the same prepacked sets also for NNPDF2.0. We have checked that results for Higgs 

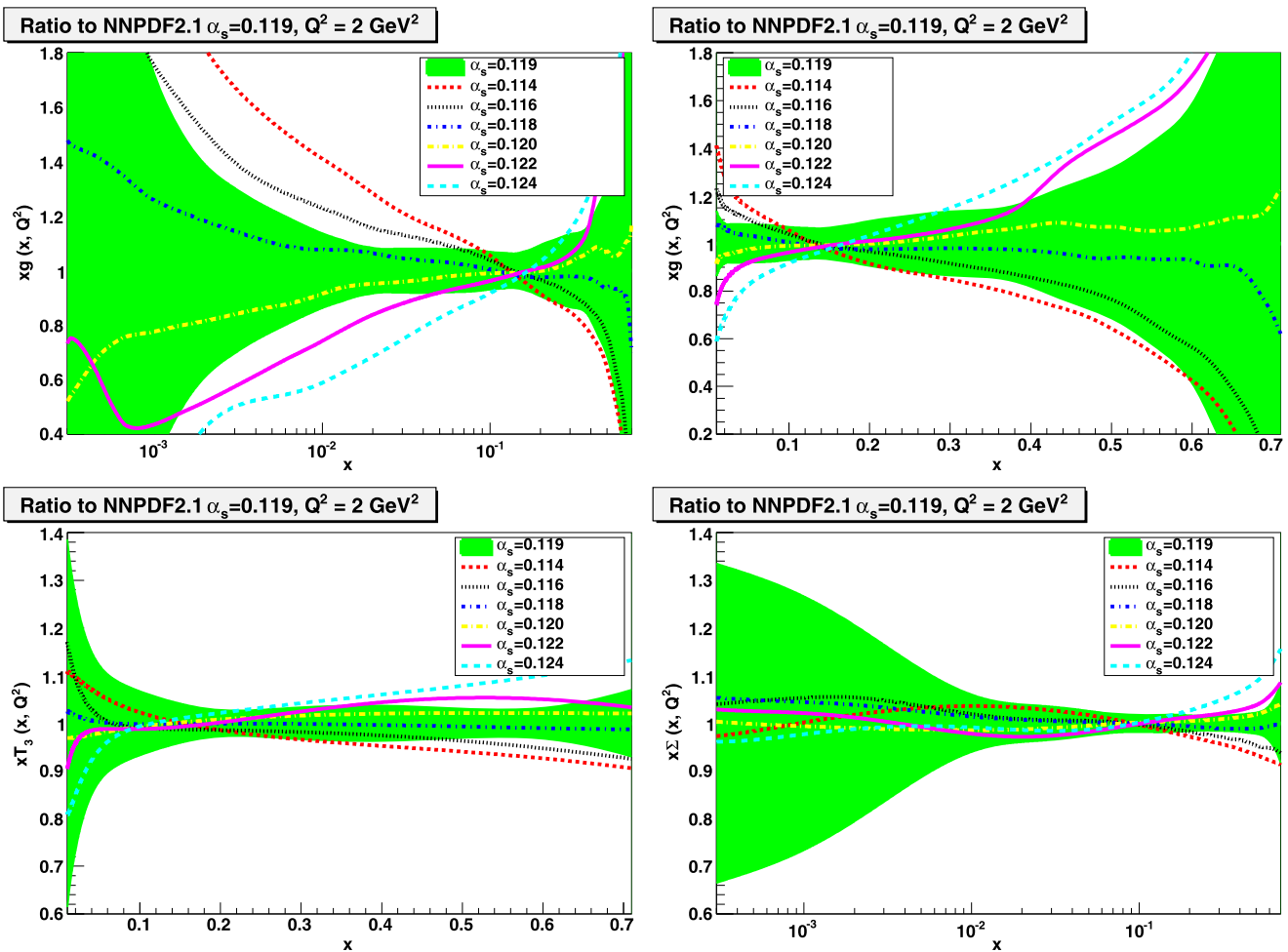

Fig. 33. Comparison between NNPDF2.1 sets with different values of the strong coupling, shown as ratios with respect the reference fit with $\alpha_{S}=0.119$. The PDFs shown are the gluon at small and large- $x$ (upper plots), the triplet at large- $x$ and the singlet at small- $x$ (lower plots).
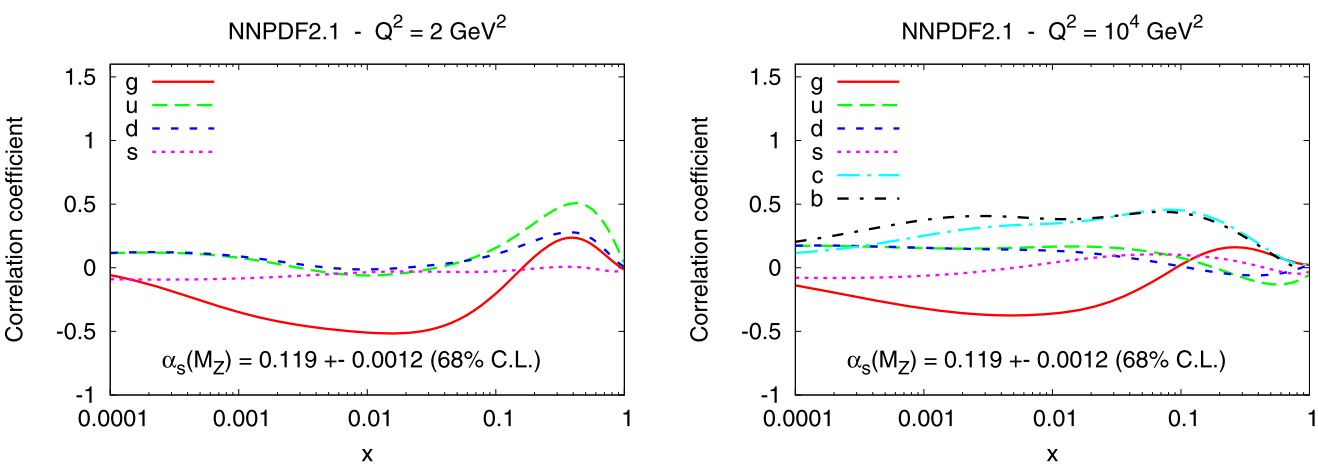

Fig. 34. Correlation coefficient between PDFs and $\alpha_{S}\left(M_{Z}\right)$ computed assuming $\alpha_{S}\left(M_{Z}\right)=0.119 \pm 0.0012$ at $68 \%$ C.L. Results are shown at low scale $\left(Q^{2}=2 \mathrm{GeV}^{2}\right.$, left $)$ and high scale $\left(Q^{2}=100 \mathrm{GeV}^{2}\right.$, right $)$.

production in gluon fusion at LHC $7 \mathrm{TeV}$ kinematics (which has rather large $\alpha_{s}$ uncertainties and correlations) become essentially independent of the number of replicas in the prepacked set provided $N_{\text {rep }} \gtrsim 100$. For smaller number of replicas there is a certain loss of accuracy, so a minimum of $N_{\text {rep }}=100$ is recommended. 


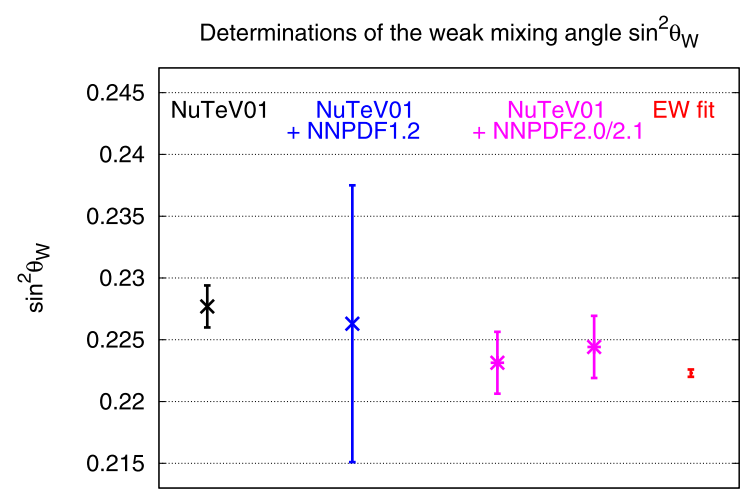

Fig. 35. Determination of the Weinberg angle from the uncorrected $\mathrm{NuTeV}$ data [66], with $\left[S^{-}\right]$correction determined from NNPDF1.2, NNPDF2.0 and NNPDF2.1. The uncertainty shown on NNPDF2.0 and NNPDF2.1 is the one- $\sigma$ PDF uncertainty only.

An important caveat in the usage of prepacked PDF sets is that some widely used codes, such as MCFM, assume the value of $\alpha_{s}$ is the same for all PDFs in a given set. Prepacked sets cannot be used with these codes. Similar prepacked PDF sets could be prepared to include the uncertainty on other physical parameters, such as heavy quark masses. An important limitation however is imposed by the current LHAPDF standard which assumes that all physical parameters except the strong coupling take the same value for all PDFs in a given set. For this reason, only $\mathrm{PDF}+\alpha_{s}$ prepacked sets are provided for the time being.

\subsection{The NuTeV anomaly}

In previous NNPDF releases $[9,10]$ we studied the implications that the determination of the strangeness asymmetry $s^{-}\left(x, Q^{2}\right)$ has on the so-called NuTeV anomaly [80]. These results are updated here. For the first moment of the strangeness asymmetry with NNPDF2.1 we find

$$
\begin{aligned}
R_{S}\left(Q^{2}\right) & \equiv 2 \frac{\int_{0}^{1} d x x s^{-}\left(x, Q^{2}\right)}{\int_{0}^{1} d x x\left(u^{-}\left(x, Q^{2}\right)+d^{-}\left(x, Q^{2}\right)\right)} \\
& =2 \frac{\left[S^{-}\right]}{\left[U^{-}+D^{-}\right]}=(1.37 \pm 0.77) \times 10^{-2} .
\end{aligned}
$$

In Fig. 35 we show the NuTeV determination of the Weinberg angle [66], uncorrected and then corrected for the strangeness asymmetry using the values from previous $[9,10]$ and the current NNPDF sets. The three corrected values are in excellent agreement with the electroweak fit and with each other, with the NNPDF2.0 and NNPDF2.1 values very close to each other, thereby showing that the impact of heavy quark mass effects on the determination of the strangeness asymmetry is very small.

\subsection{Comparison with present and future HERA $F_{2}^{c}$ and $F_{L}$ data}

In conclusion, we look at NNPDF2.1 predictions for $F_{2}^{c}$ and $F_{L}$, which are especially sensitive to the treatment of heavy quark mass effects. For instance, heavy quark mass corrections to $F_{L}$ for $Q^{2} \leqslant 20 \mathrm{GeV}^{2}$ are larger than $30 \%$, see Fig. 3. In Fig. 36 we compare to the best fit result 

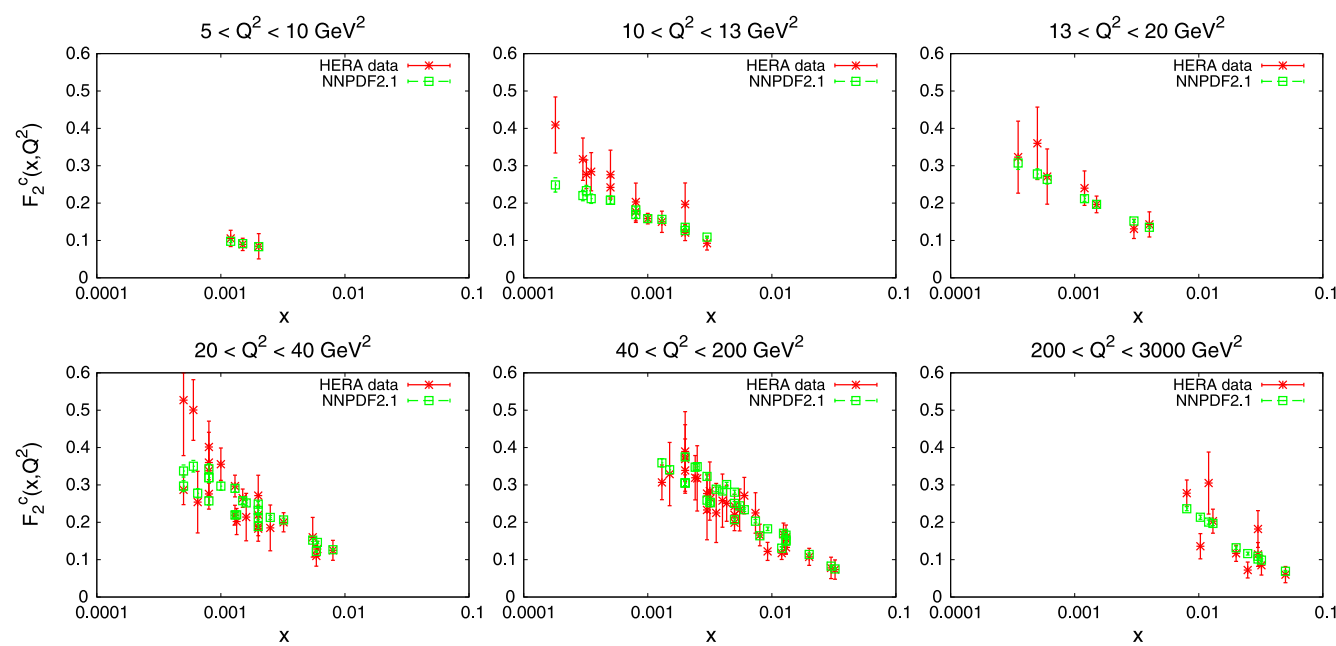

Fig. 36. Comparison between the H1 and ZEUS $F_{2}^{c}$ data included in the present analysis and the NNPDF2.1 best fit. The data uncertainty includes statistical and systematic errors added in quadrature, while the theoretical uncertainty is the PDF uncertainty only.
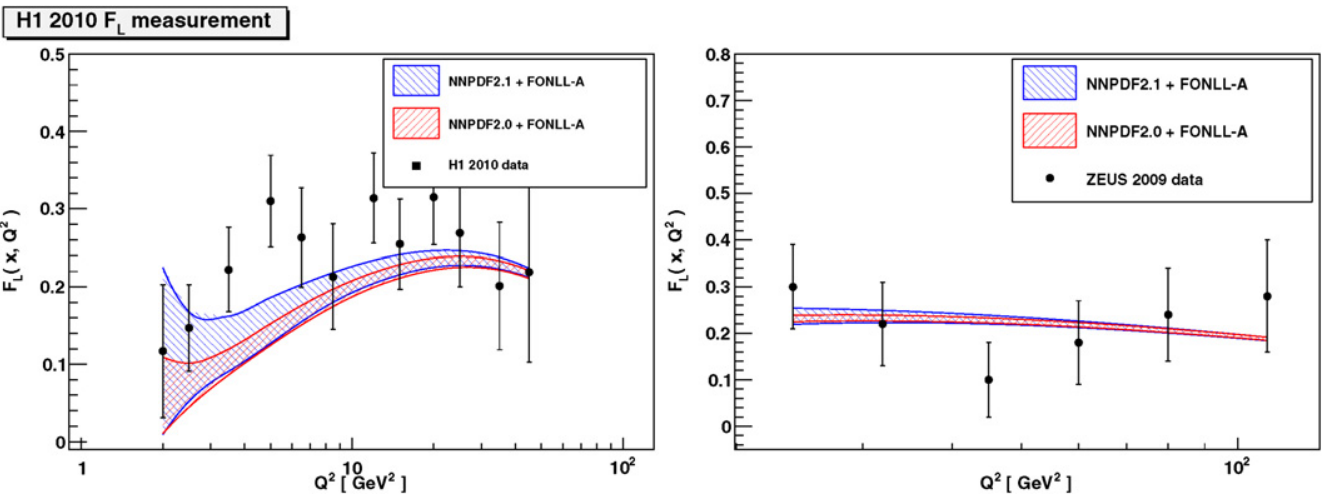

Fig. 37. Comparison between H1 [82] (left) and ZEUS [81] (right) $F_{L}$ data and NNPDF2.1 predictions. Predictions using NNPDF2.0 with the FONLL-A are also shown.

the HERA $F_{2}^{c}$ data which have been included in the present analysis: in general the agreement is rather good, though the lowest $Q^{2}$ and $x$ bins have been removed from the fitted dataset, because $\mathcal{O}\left(\alpha_{s}^{2}\right)$ heavy quark corrections, not included in the present analysis, are large there [11]. The NNPDF2.1 predictions for $F_{L}\left(x, Q^{2}\right)$ is compared to published ZEUS [81] and H1 [82] data in Fig. 37. Note that while the H1 data are included in the fit, they have rather large uncertainties and thus carry very little weight in the global fit. Predictions obtained using NNPDF2.0 PDFs, but including heavy quark mass effects in the computation of the structure function through FONLL-A are also shown. This comparison is particularly interesting, because heavy quark mass effects are quite large especially at low- $Q^{2}$ : this correction is included here with both sets, though NNPDF2.0 PDFs were determined without it. The good agreement between results found using the two sets shows that NNPDF2.0 PDFs are quite accurate despite the lack of heavy quark mass corrections in the fit. 


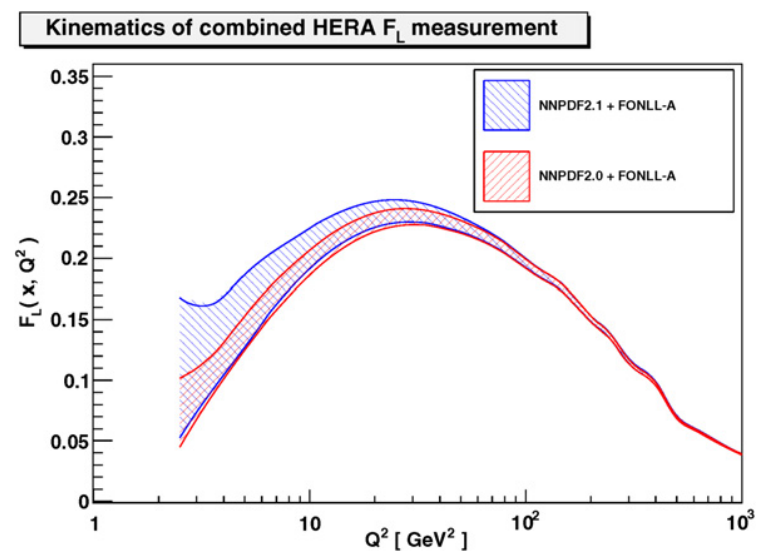

Fig. 38. The NNPDF2.1 predictions for $F_{L}$ in the kinematics of upcoming combined HERA data. Predictions using NNPDF2.0 with the FONLL-A are also shown.

We now turn to the predictions in view of upcoming combined HERA data. In Fig. 38 we provide the NNPDF2.1 predictions for $F_{L}$ in the kinematic region of the upcoming combined HERA data. ${ }^{7}$ The increase in uncertainty at small- $x$ is driven by the larger uncertainty on the gluon at small- $x$, as seen in Fig. 12. We also show the results using FONLL-A with both NNPDF2.0 and NNPDF2.1 input PDFs. The NNPDF2.1 results have been compared with preliminary combined HERA $F_{L}$ dataset in Ref. [83].

In conclusion, we give predictions for $F_{2}^{c}$ in Fig. 39 in the range of upcoming combined HERA data. ${ }^{8}$ These predictions are obtained with heavy quark mass effects included up to $\mathcal{O}\left(\alpha_{s}^{2}\right)$ through the FONLL-B scheme, but using input PDFs determined with $\mathcal{O}\left(\alpha_{s}\right)$ heavy quark mass corrections. The fact that FONLL-A and FONLL-B coincide for moderate and large values of $Q^{2}$, where the $F_{2}^{c}$ data included in NNPDF2.1 lie, justifies the use of FONLL-B to extrapolate to the low- $Q^{2}$ region with the same input PDF set. PDF uncertainties at small- $x$ and $Q^{2}$ are rather large, suggesting that the combined HERA $F_{2}^{c}$ data will impose severe constraints on the small- $x$ gluon; comparison with preliminary data [83] suggests that the NNPDF2.1 will be in very good agreement with the HERA data down to the smallest values of $Q^{2}$.

\section{Heavy quark mass dependence}

We will now discuss the dependence of PDFs on the values of heavy quark masses $m_{c}$ and $m_{b}$, and present some preliminary investigations on uncertainties related to heavy quark masses, along the lines of the recent detailed study by the MSTW group [84]; as an outcome of this analysis we will present NNPDF2.1 sets with varying $m_{c}$ and $m_{b}$ masses. We will first discuss how different features of the NNPDF2.1 PDFs depend on the values of $m_{c}$ and $m_{b}$. We then briefly investigate the dependence on $m_{c}$ of some LHC observables and summarize how uncertainties on heavy quark masses can be treated in the Monte Carlo approach. Finally, as an example of a

7 We thank S. Glazov for providing us this information.

8 We thank K. Lipka for providing these plots. 


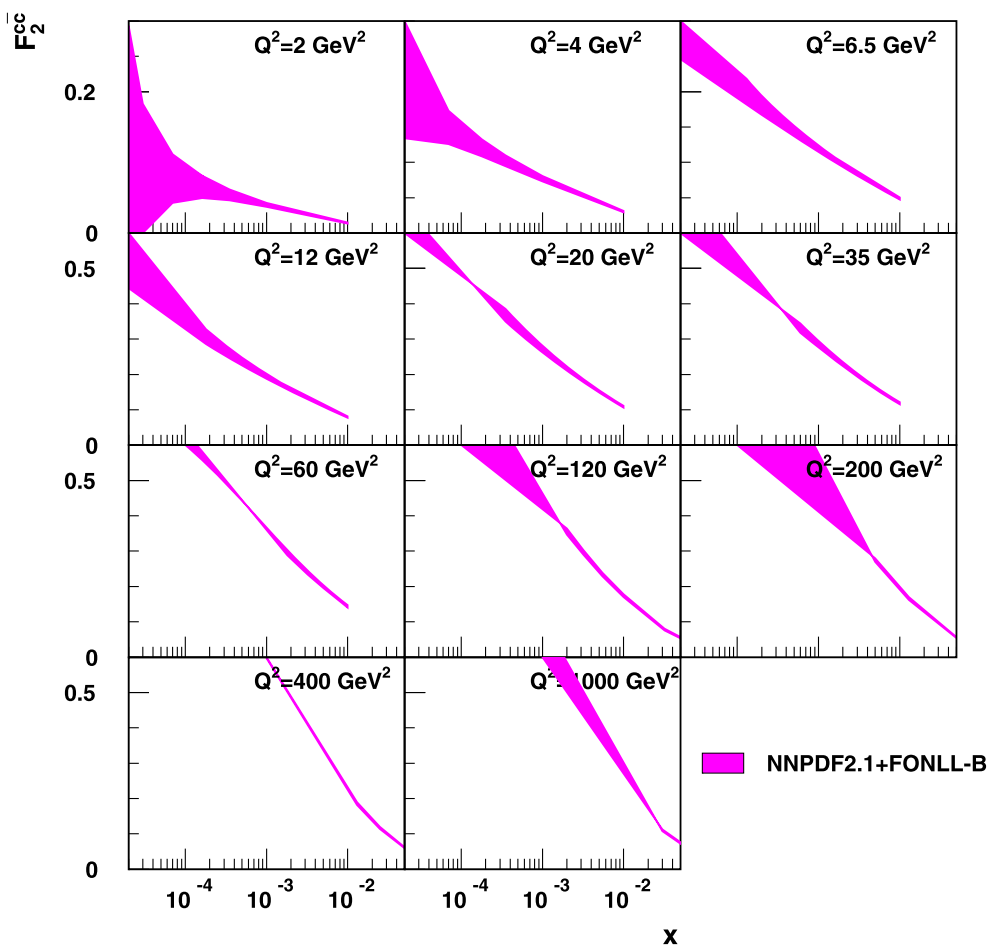

Fig. 39. NNPDF2.1 predictions for $F_{2}^{c}$ with heavy quark mass effects included up to $\mathcal{O}\left(\alpha_{s}^{2}\right)$ through the FONLL-B scheme in the kinematic region of upcoming combined HERA data.

Table 10

The default values of the heavy quark masses used in NNPDF2.1 and in several recent PDF sets.

\begin{tabular}{lll}
\hline & $m_{c}[\mathrm{GeV}]$ & $m_{b}[\mathrm{GeV}]$ \\
\hline NNPDF2.1 & $\sqrt{2}$ & 4.75 \\
NNPDF2.0 [10] & $\sqrt{2}$ & 4.3 \\
CT10 [16] & 1.30 & 4.75 \\
MSTW2008 [84] & 1.40 & 4.75 \\
ABKM09 [24] & 1.50 & 4.50 \\
HERAPDF1.0 [25] & 1.40 & 4.75 \\
\hline
\end{tabular}

possible application we evaluate the combined PDF $+m_{b}$ uncertainty for MSSM Higgs boson production.

\subsection{Dependence of PDFs on heavy quark masses}

The default value of heavy quark masses used so far are summarized and compared to those of other PDF sets in Table 10. The dependence of PDFs on the heavy quark masses is studied by repeating the NNPDF2.1 fit with different mass values. In particular, we have repeated the reference fit for charm quark masses of $1.5,1.6$ and $1.7 \mathrm{GeV}$ as well as for bottom masses of $4.25,4.5,5.0$ and $5.25 \mathrm{GeV}$. It is important to observe that at the order at which we are working, 

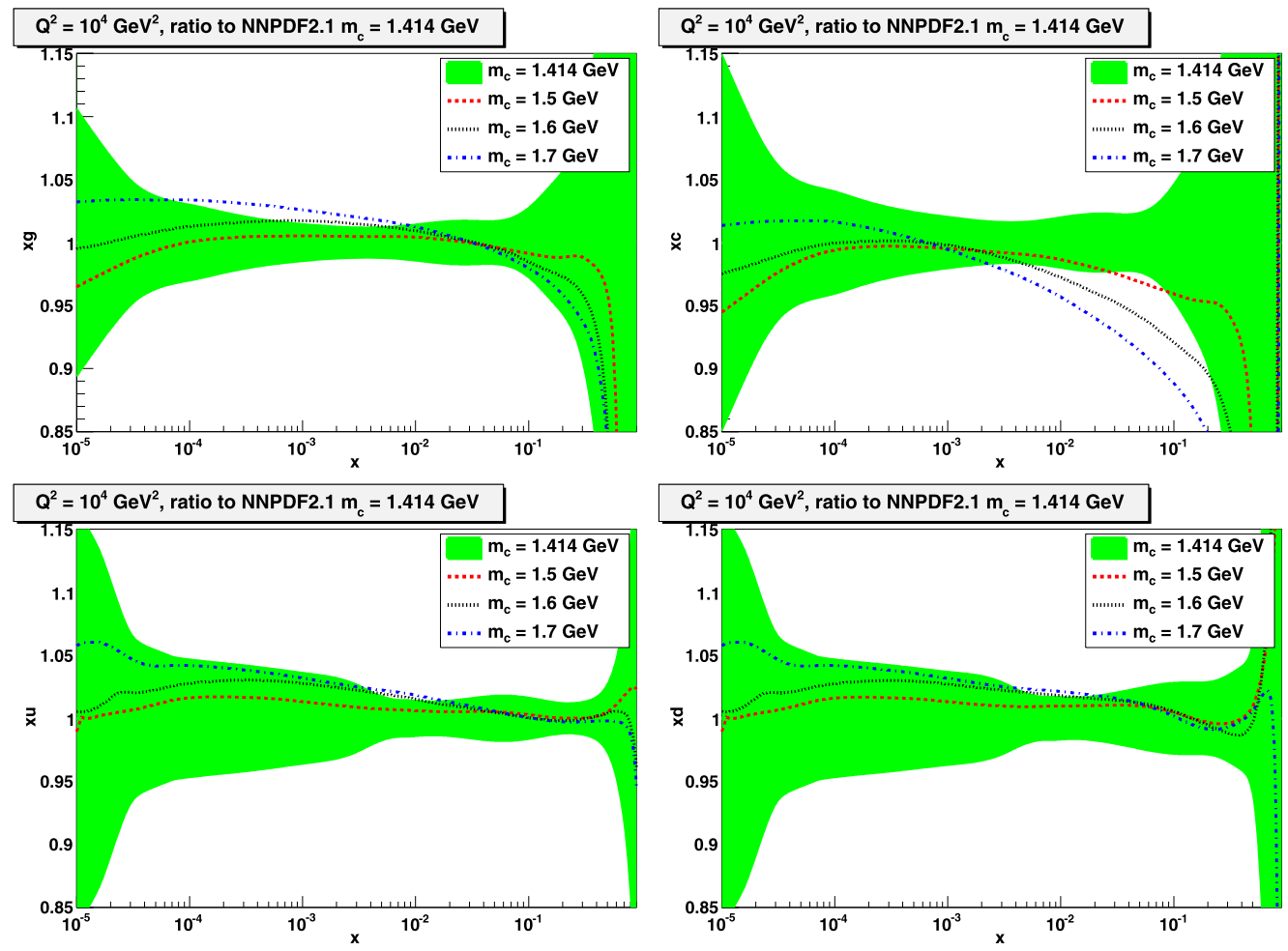

Fig. 40. Ratio of NNPDF2.1 PDFs obtained for different values of the charm quark mass to the reference NNPDF2.1 set at $Q^{2}=10^{4} \mathrm{GeV}^{2}$. Top left: gluon; top right: charm; bottom left: up; bottom right: down.

the perturbative definition of the heavy quark mass is immaterial: indeed different definitions (such as, for example, the pole and $\overline{\mathrm{MS}}$ mass definitions) differ by terms of $\mathcal{O}\left(\alpha_{S}\right)$. However, we are including heavy quark mass corrections up to $\mathcal{O}\left(\alpha_{s}\right)$ only, so the difference is subleading (it becomes relevant once one includes $\mathcal{O}\left(\alpha_{s}^{2}\right)$ heavy quark corrections, for example using the FONLL-C scheme). Therefore, the value of the quark mass in our PDF determination (as well as in other PDF determinations based on an NLO ACOT treatment of heavy quarks, such as $\mathrm{CT} / \mathrm{CTEQ}$ ) can be equivalently interpreted as, say, a pole mass or an $\overline{\mathrm{MS}}$ mass. The $\overline{\mathrm{MS}}$ mass is better known, and it has been recently shown [85] to lead to perturbatively more stable results for deep-inelastic structure functions.

Results are shown in Figs. 40, 41 where the ratios of PDFs for different values of $m_{c}$ and $m_{b}$ to the reference NNPDF2.1 fit are plotted as a function of $x$ for $Q^{2}=10^{4} \mathrm{GeV}^{2}$. The dependence of the heavy quark PDFs on the value of the mass is easily understood: heavy quark PDFs are generated radiatively, and assumed to vanish at a scale equal to their mass. Therefore, a lower mass value corresponds to a longer evolution length and thus to a larger heavy quark PDF, and conversely. Thus, if one allowed [86] for an "intrinsic" [87] heavy component (i.e. for a nonvanishing initial condition) this uncertainty would be absorbed in the initial intrinsic heavy PDF. Because of the momentum sum rule, if the charm PDF becomes larger, other PDFs are accordingly smaller (and conversely). For bottom in principle the same mechanism is at work, but in practice the effect on all other PDFs is negligible. 
Table 11

LHC standard candles at $\sqrt{s}=7 \mathrm{TeV}$ (upper table) and $14 \mathrm{TeV}$ (lower table) obtained using NNPDF2.1 fits with different values of the charm mass $m_{c}$; the values in the top line of each table are the same given in Section 5.1. The bottom line of each table gives the correlation coefficient between the observable and the mass.

\begin{tabular}{|c|c|c|c|c|c|}
\hline LHC $7 \mathrm{TeV}$ & $W^{+} B_{l v}[\mathrm{nb}]$ & $W^{-} B_{l v}[\mathrm{nb}]$ & $Z^{0} B_{l \bar{l}}[\mathrm{nb}]$ & $t \bar{t}[\mathrm{pb}]$ & $g g \rightarrow H[\mathrm{pb}]$ \\
\hline $\begin{array}{l}m_{c}= \\
1.414 \mathrm{GeV}\end{array}$ & $5.99 \pm 0.14$ & $4.09 \pm 0.09$ & $\begin{array}{l}0.932 \pm \\
0.020\end{array}$ & $170 \pm 5$ & $11.64 \pm 0.17$ \\
\hline $\begin{array}{l}m_{c}= \\
1.5 \mathrm{GeV}\end{array}$ & $6.06 \pm 0.17$ & $4.14 \pm 0.12$ & $\begin{array}{l}0.943 \pm \\
0.024\end{array}$ & $169 \pm 6$ & $11.65 \pm 0.25$ \\
\hline $\begin{array}{l}m_{c}= \\
1.6 \mathrm{GeV}\end{array}$ & $6.11 \pm 0.14$ & $4.17 \pm 0.10$ & $\begin{array}{l}0.951 \pm \\
0.020\end{array}$ & $167 \pm 6$ & $11.70 \pm 0.21$ \\
\hline $\begin{array}{l}m_{c}= \\
1.7 \mathrm{GeV}\end{array}$ & $6.14 \pm 0.14$ & $4.19 \pm 0.09$ & $\begin{array}{l}0.956 \pm \\
0.019\end{array}$ & $166 \pm 5$ & $11.71 \pm 0.22$ \\
\hline$\rho\left[\sigma, m_{c}\right]$ & 0.44 & 0.41 & 0.48 & -0.31 & 0.16 \\
\hline$\underline{\mathrm{LHC}} 14 \mathrm{TeV}$ & $W^{+} B_{l v}[\mathrm{nb}]$ & $W^{-} B_{l v}[\mathrm{nb}]$ & $Z^{0} B_{l \bar{l}}[\mathrm{nb}]$ & $t \bar{t}[\mathrm{pb}]$ & $g g \rightarrow H[\mathrm{pb}]$ \\
\hline $\begin{array}{l}m_{c}= \\
1.414 \mathrm{GeV}\end{array}$ & $12.00 \pm 0.27$ & $8.84 \pm 0.17$ & $1.99 \pm 0.036$ & $946 \pm 19$ & $37.50 \pm 0.40$ \\
\hline $\begin{array}{l}m_{c}= \\
1.5 \mathrm{GeV}\end{array}$ & $12.01 \pm 0.31$ & $8.94 \pm 0.22$ & $2.01 \pm 0.04$ & $942 \pm 24$ & $37.62 \pm 0.62$ \\
\hline $\begin{array}{l}m_{c}= \\
1.6 \mathrm{GeV}\end{array}$ & $12.24 \pm 0.28$ & $9.02 \pm 0.20$ & $2.03 \pm 0.04$ & $939 \pm 22$ & $37.90 \pm 0.55$ \\
\hline $\begin{array}{l}m_{c}= \\
1.7 \mathrm{GeV}\end{array}$ & $12.37 \pm 0.28$ & $9.10 \pm 0.18$ & $2.05 \pm 0.04$ & $935 \pm 19$ & $38.15 \pm 0.58$ \\
\hline$\rho\left[\sigma, m_{c}\right]$ & 0.48 & 0.50 & 0.56 & -0.19 & 0.41 \\
\hline
\end{tabular}

\subsection{Mass uncertainties and LHC observables}

The dependence of light quark distributions and the gluon on the charm mass displayed in Fig. 40 is strong enough to affect the LHC standard candles studied in Section 5.1 at the percent level or more, as was discovered relatively recently [15]. On the other hand, the dependence on the bottom mass of all PDFs but $b$ itself is below the percent level, so only observables which depend on bottom are affected significantly: an example will be considered in Section 6.4 below.

Values of LHC standard candles computed as in Section 5.1 but using the PDF sets with different values of the charm mass of Fig. 40 are collected in Table 11, and shown in Fig. 42. For completeness, we also give in Table 12 standard candles at $7 \mathrm{TeV}$ for several values of the $b$ mass. The variation of all standard candles is at the percent level for charm mass variations of order of $10 \%$. It is interesting to observe that the variation seen when modifying subleading charm mass terms is (recall Table 9) of the same order of magnitude and in fact somewhat larger. This suggests that even though PDF uncertainties on standard candles are still dominant at present, theoretical uncertainties related to the treatment of charm will become relevant and possibly dominant as soon as PDF uncertainties are reduced by a factor of two or three.

\subsection{Combined $\mathrm{PDF}+m_{h}$ uncertainties and correlations}

Uncertainties which combine PDF uncertainty and heavy quark mass dependence are easily determined in a Monte Carlo approach, provided that PDF sets for several values of the quark 

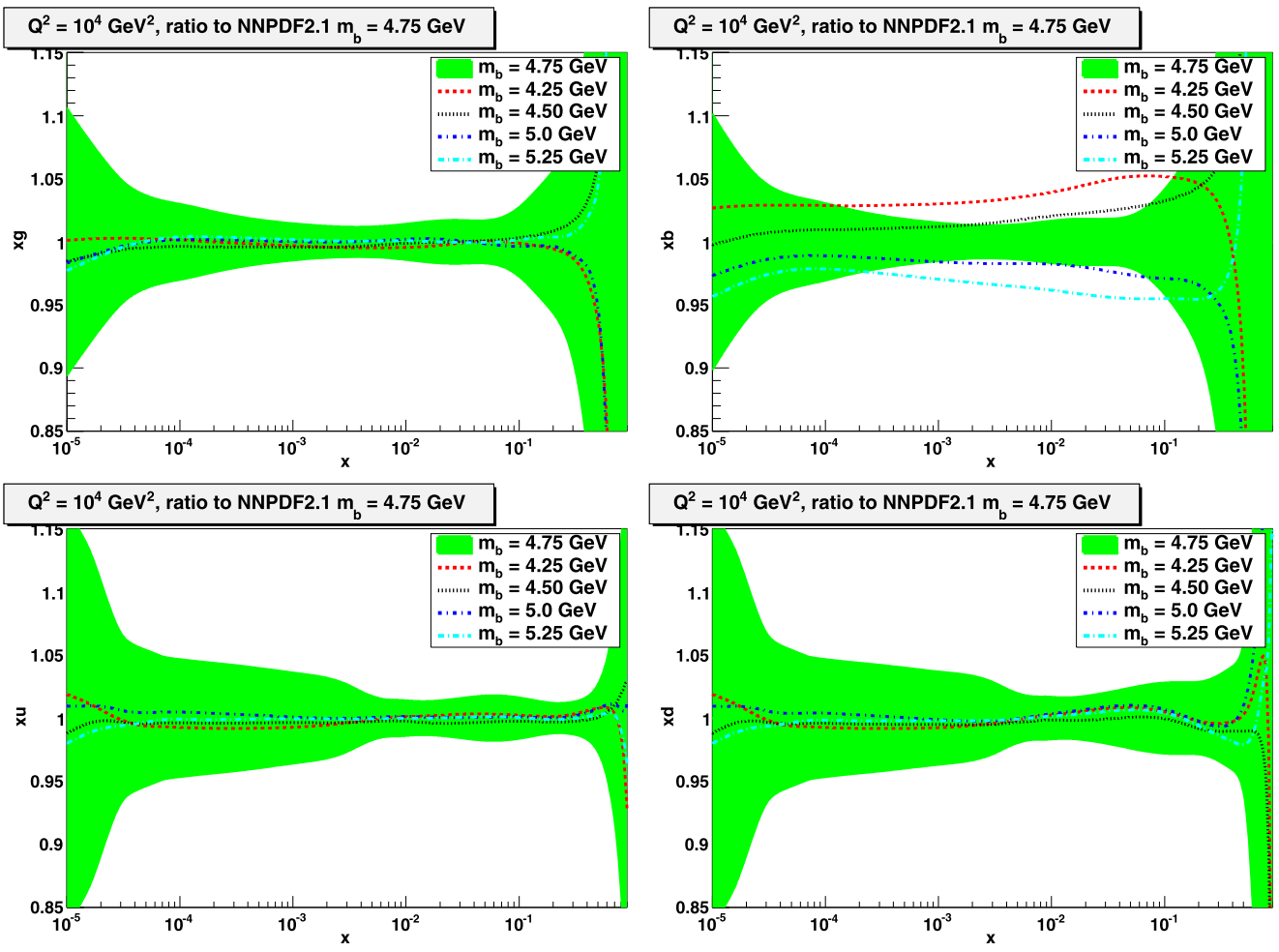

Fig. 41. Ratio of NNPDF2.1 PDFs obtained for different values of the bottom quark mass to the reference NNPDF2.1 set at $Q^{2}=10^{4} \mathrm{GeV}^{2}$. Top left: gluon; top right: bottom; bottom left: up; bottom right: down.

Table 12

LHC standard candles at $\sqrt{s}=7 \mathrm{TeV}$ obtained using NNPDF2.1 fits with different values of the bottom mass $m_{b}$; the values in the top line of each table are the same given in Section 5.1.

\begin{tabular}{llllll}
\hline & $W^{+} B_{l v}[\mathrm{nb}]$ & $W^{-} B_{l v}[\mathrm{nb}]$ & $Z^{0} B_{l \bar{l}}[\mathrm{nb}]$ & $t \bar{t}[\mathrm{pb}]$ & $g g \rightarrow H[\mathrm{pb}]$ \\
\hline$m_{b}=4.25 \mathrm{GeV}$ & $5.97 \pm 0.12$ & $4.07 \pm 0.08$ & $0.930 \pm 0.016$ & $170 \pm 6$ & $11.58 \pm 0.26$ \\
$m_{b}=4.5 \mathrm{GeV}$ & $5.95 \pm 0.21$ & $4.07 \pm 0.11$ & $0.928 \pm 0.025$ & $171 \pm 7$ & $11.64 \pm 0.18$ \\
$m_{b}=4.75 \mathrm{GeV}$ & $5.99 \pm 0.14$ & $4.09 \pm 0.09$ & $0.932 \pm 0.020$ & $170 \pm 5$ & $11.64 \pm 0.17$ \\
$m_{b}=5.0 \mathrm{GeV}$ & $5.99 \pm 0.12$ & $4.11 \pm 0.07$ & $0.932 \pm 0.016$ & $170 \pm 5$ & $11.64 \pm 0.17$ \\
$m_{b}=5.25 \mathrm{GeV}$ & $5.98 \pm 0.11$ & $4.10 \pm 0.07$ & $0.930 \pm 0.015$ & $171 \pm 6$ & $11.66 \pm 0.18$ \\
\hline
\end{tabular}

masses are available. Here we provide several such sets, and more with a finer mass spacing and wider range will be made available in the future (such as already available for the MSTW08 sets [84]). Given sets of PDF replicas labeled by heavy quark mass values, such that $\operatorname{PDF}^{\left(k_{i j}, i, j\right)}$ is the $k_{i j}$-th replica of the PDF set with heavy quark mass values $m_{c}^{(i)}$ and $m_{b}^{(j)}$, the mean value of any observable $\mathcal{F}$ is

$$
\langle\mathcal{F}\rangle_{\text {rep }}=\frac{1}{N_{\text {rep }}} \sum_{i=1}^{N_{m_{c}}} \sum_{j=1}^{N_{m_{b}}} \sum_{k_{i j}=1}^{N_{\text {rep }}^{(i, j)}} \mathcal{F}\left(\mathrm{PDF}^{\left(k_{i j}, i, j\right)}, m_{c}^{(i)}, m_{b}^{(j)}\right),
$$



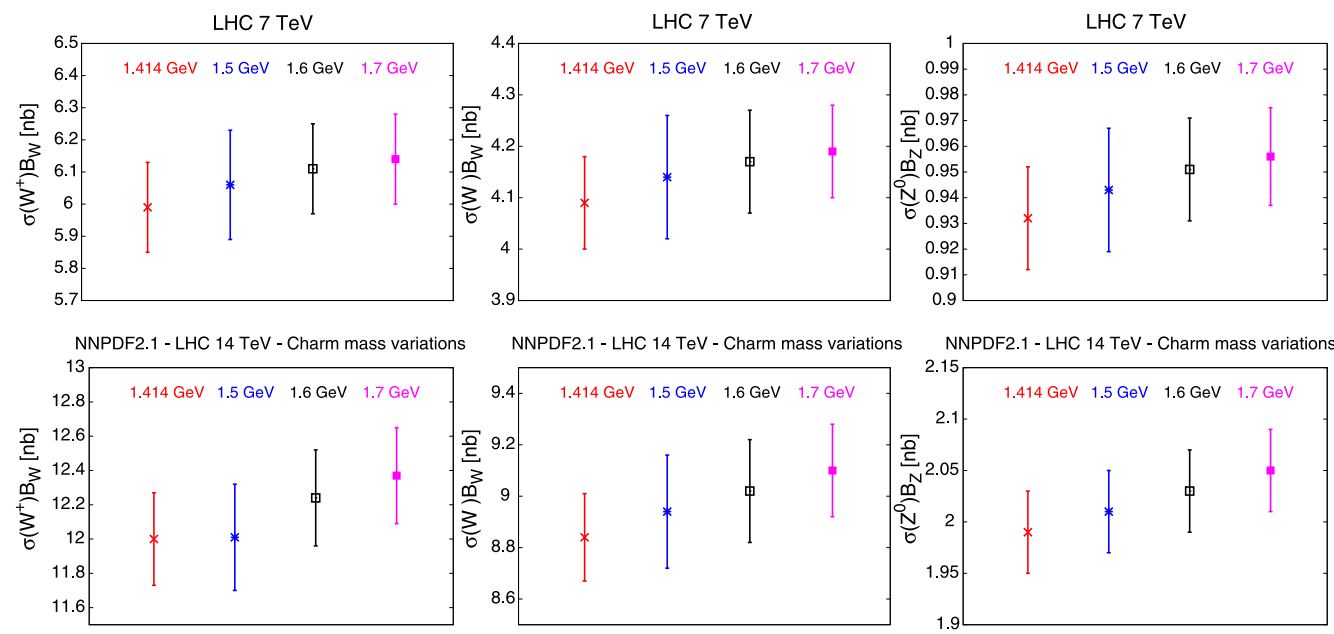

Fig. 42. Graphical representation of the results of Table 11.

where

$$
N_{\text {rep }}=\sum_{i=1}^{N_{m_{c}}} \sum_{j=1}^{N_{m_{b}}} N_{\text {rep }}^{(i, j)}
$$

is the total number of replicas, and $N_{\text {rep }}^{(i, j)}$ are distributed according to a two-dimensional Gaussian distribution with mean $\left(m_{c}^{(0)}, m_{b}^{(0)}\right)$ and width $\left(\delta m_{c}, \delta m_{b}\right)$ (assuming the values of charm and bottom masses are uncorrelated):

$$
N_{\text {rep }}^{(i, j)} \propto \exp \left(-\frac{\left(m_{c}^{(i)}-m_{c}^{(0)}\right)^{2}}{2 \delta_{m_{c}}^{2}}-\frac{\left(m_{b}^{(j)}-m_{b}^{(0)}\right)^{2}}{2 \delta_{m_{b}}^{2}}\right) .
$$

The combined PDF $+m_{h}$ uncertainty is then the standard deviation of the observable over the replica sample:

$$
\delta_{\mathrm{PDF}+m_{h}} \mathcal{F}=\sqrt{\left\langle\mathcal{F}^{2}\right\rangle-\langle\mathcal{F}\rangle^{2}},
$$

where averages over replicas are to be understood as in Eq. (37). Of course, a different probability distribution (possibly including a correlation between heavy quark mass values) could be assumed instead of Eq. (39).

We can easily compute the correlation between PDFs and heavy flavor masses $m_{h}$ :

$$
\rho\left[m_{h}, \operatorname{PDF}\left(x, Q^{2}\right)\right]=\frac{\left\langle m_{h} \operatorname{PDF}\left(x, Q^{2}\right)\right\rangle_{\text {rep }}-\left\langle m_{h}\right\rangle_{\text {rep }}\left\langle\operatorname{PDF}\left(x, Q^{2}\right)\right\rangle_{\text {rep }}}{\sigma_{m_{h}} \sigma_{\operatorname{PDF}\left(x, Q^{2}\right)}},
$$

where averages over replicas are to be understood in the sense of Eq. (37). The correlation (41), computed assuming $m_{c}=1.55 \pm 0.15 \mathrm{GeV}$ and $m_{b}=4.75 \pm 0.25 \mathrm{GeV}$, is displayed in Fig. 43, as a function of $x$ for $Q^{2}=10^{4} \mathrm{GeV}^{2}$. As discussed in Section 6.1, as the mass is increased the corresponding heavy quark PDF is reduced, i.e. the PDF is strongly anticorrelated with its mass. As a consequence of the heavy quark suppression other PDFs are enhanced and this appears as a positive correlation, though the effect for bottom is negligibly small. Correlations between $m_{h}$ 

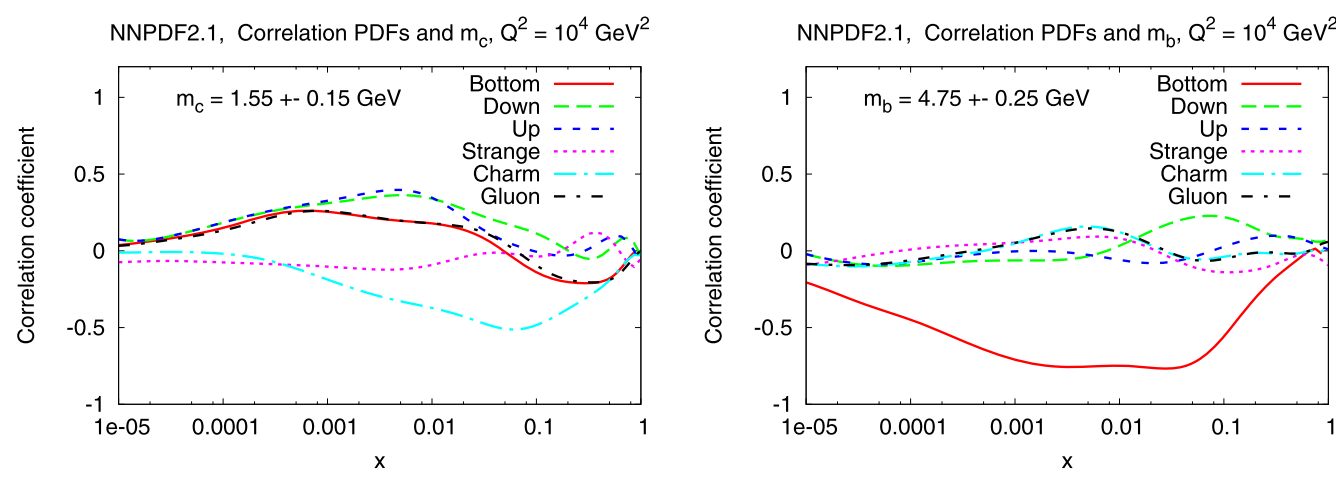

Fig. 43. Correlation between PDFs and the heavy quark masses at a typical LHC scale $Q^{2}=10^{4} \mathrm{GeV}^{2}: \mathrm{charm}^{\mathrm{mass}}$ variations (left plot) and bottom mass variations (right plot). These correlations quantify the qualitative behavior observed in Figs. 40, 41.
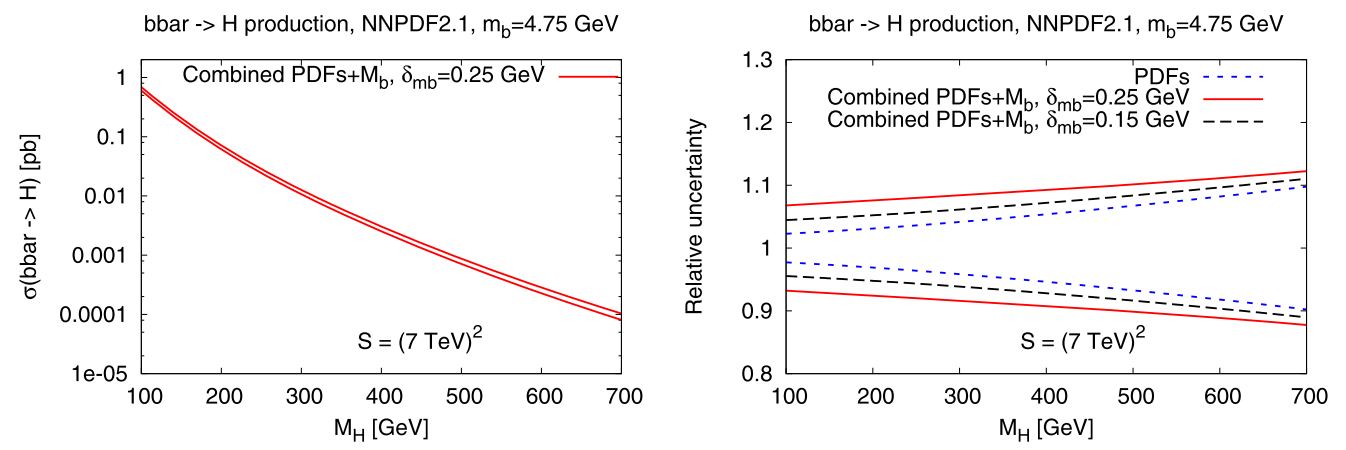

Fig. 44. Combined PDF $+m_{b}$ uncertainties on the total cross-section for $b \bar{b} \rightarrow H$ Higgs production at the LHC 7 TeV with $m_{b}=4.75 \mathrm{GeV}$. The absolute cross-section (left) and relative uncertainty (right) are shown. In the right plot, the PDF-only uncertainty is compared to the combined PDF $+m_{b}$ uncertainty with $\delta_{m_{b}}=0.15$ or $\delta_{m_{b}}=0.25$ at one- $\sigma$.

and physical observables can be computed analogously. They are given in Table 11 and can be used for a quick estimate of the corresponding uncertainty.

\subsection{The combined $\mathrm{PDF}+m_{b}$ uncertainties in MSSM $b \bar{b} \rightarrow H$ production}

As an illustration of the procedure to combine PDF and $m_{b}$ uncertainties discussed in Section 6.3, we have evaluated the combined uncertainty for Higgs production in association with bottom quarks [88]. Higgs production via bottom fusion is enhanced in the MSSM in large $\tan \beta$ scenarios as compared to the SM, so this channel is important for supersymmetry searches.

We have used the code of Ref. [88] to computed the $b \bar{b} \rightarrow H$ cross-section to NLO in the MSSM, using the NNPDF2.1 sets with variable $m_{b}$ of Section 6.1. For other physical parameters we take the default values. Results are shown in Fig. 44 as a function of the Higgs mass for LHC $7 \mathrm{TeV}$, with two different uncertainty ranges for the bottom mass (the current PDG [65] quotes ${ }_{-0.07}^{+0.17}$ as uncertainty on the $\overline{\mathrm{MS}} b$ mass). Even with the smaller range the mass uncertainty is not negligible in comparison to the PDF uncertainty.

It would be interesting to extend this analysis to several LHC processes which are expected to depend significantly on heavy quark masses, such as for instance $t$-channel single-top production. 
Table 13

NNPDF2.1 PDF sets with maximum fixed flavor number of active quarks. In all cases, the number of flavours of the reference NNPDF2.1 PDF set is frozen at $Q_{\text {match }}^{2}$, and PDFs are then evolved upwards with a fixed number of flavours. For each set, the values of the heavy quark masses, matching scale, and strong coupling at the matching scale and at $Q^{2}=M_{z}^{2}$ are shown.

\begin{tabular}{lllllll}
\hline LHAPDF name & $N_{f}^{\max }$ & $m_{c}[\mathrm{GeV}]$ & $m_{b}[\mathrm{GeV}]$ & $Q_{\text {match }}[\mathrm{GeV}]$ & $\alpha_{s}\left(Q_{\text {match }}\right)$ & $\alpha_{s}^{\left(N_{f}^{\max }\right)}\left(M_{Z}\right)$ \\
\hline NNPDF21_100.LHgrid & 6 & $\sqrt{2}$ & 4.75 & - & - & 0.11900 \\
NNPDF21_FFN_NF3_100.LHgrid & 3 & - & - & $\sqrt{2}$ & 0.359912 & 0.10585 \\
NNPDF21_FFN_NF4_100.LHgrid & 4 & $\sqrt{2}$ & - & 4.75 & 0.218200 & 0.11343 \\
NNPDF21_FFN_NF5_100.LHgrid & 5 & $\sqrt{2}$ & 4.75 & 175 & 0.108283 & 0.11900 \\
\hline
\end{tabular}

\section{PDFs with fixed flavor number}

The NNPDF2.1 PDFs discussed so far are determined in a factorization scheme in which the number of flavors depends on the scale, and in particular it varies from $N_{f}=3$ when $Q^{2}<m_{c}^{2}$ to $N_{f}=6$ when $Q^{2}>m_{t}^{2}$. Such a scheme is advantageous in that it includes terms to all orders in $\alpha_{s}$ up to the desired logarithmic order (NLO in our case) both for light and for heavy quarks, while in a general-mass scheme such as the FONLL-A scheme used here heavy quark mass terms are also included up to some fixed order in $\alpha_{s}$. Indeed, given that the charm mass is of the same order as the starting scale of perturbative evolution at which PDFs are parametrized (in fact, they coincide for the default NNPDF2.1 set), the resummation to all orders in $\alpha_{s}$ of charm mass logarithms of the form $\ln Q^{2} / m_{c}^{2}$ is as important as that for any other parton distribution. However, the LO and NLO resummation of logarithms related to heavier flavors is usually rather less important at scales relevant for LHC phenomenology, especially for top, but in practice sometimes also for bottom. In these cases, PDF sets in which the maximum number of flavors is fixed at some value lower than $N_{f}^{\max }=6$ may lead to equally accurate results. Furthermore, use of these sets is necessary in conjunction with matrix elements computed with a number of active flavor smaller than six, such as single top production [89], Higgs production in association with bottom quarks [90], as well as with Monte Carlo codes based on similar computations, such as the HVQDIS [91] Monte Carlo, widely used for the analysis of $F_{2}^{c}$, which is based on the $N_{f}=3$ scheme computation of the observable.

With these motivations, we have constructed PDF sets in which the maximum number of flavors is $N_{f}=3, N_{f}=4$ and $N_{f}=5$ (see Table 13 ). These are simply constructed by freezing the number of flavors at some scale $Q_{\text {match }}^{2}$ which is thus viewed as a matching scale between a scheme in which the number of flavors depends on scale (for $Q^{2}<Q_{\text {match }}^{2}$ ) and a scheme in which the number of flavors is fixed (for $Q^{2}>Q_{\text {match }}^{2}$ ). We will refer to these as Fixed-Flavor Number (FFN) PDFs, though this is strictly speaking a misnomer except in the $N_{f}=3$ case: for instance, below the top threshold the default NNPDF2.1 set and the $N_{f}=5$ set are identical. Note that if these PDFs are to be used with matrix elements computed with the given number of flavors, the strong coupling must be consistently determined with the same fixed number of flavor, lest a spurious $N_{f}$ dependence be introduced in physical observables (see Ref. [92], where the effect of an incorrect choice in this respect is also estimated).

In order to illustrate the differences between these PDFs in Fig. 45 we compare the FFN PDFs in the $N_{f}=3$ and $N_{f}=4$ to the reference NNPDF2.1 PDFs at the scale $Q^{2}=10^{2} \mathrm{GeV}^{2}$. Differences stem both from the milder evolution due to the reduced number of quark flavors and 

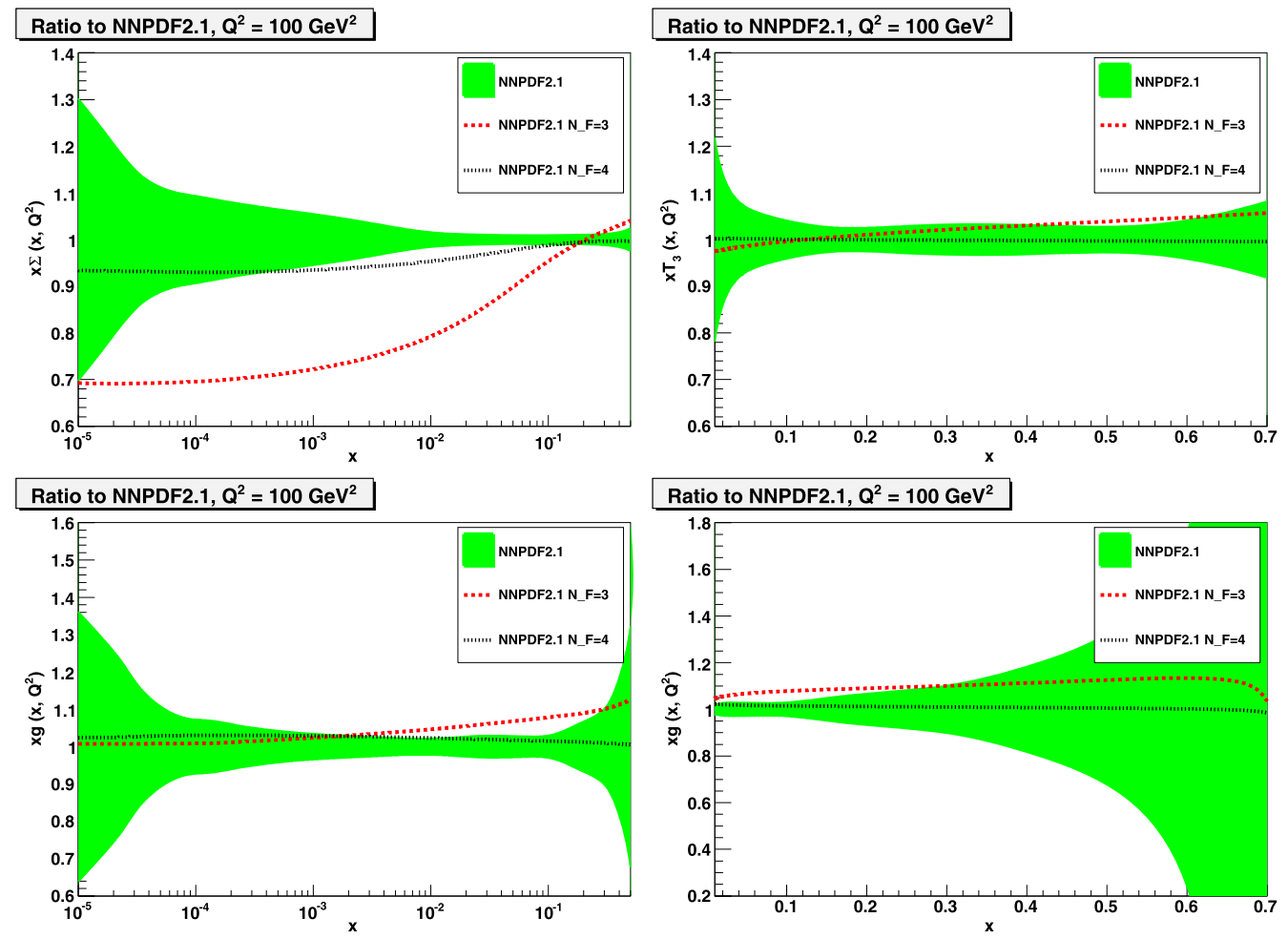

Fig. 45. Comparison between the default NNPDF2.1 set and the $N_{f}=3$ and $N_{f}=4$ FFN scheme sets at the scale $Q^{2}=10^{2} \mathrm{GeV}^{2}$. Results are shown as ratios to the default set. The singlet and the triplet (top) and the gluon in a linear and logarithmic scale (bottom) are shown.

from the correspondingly smaller value of $\alpha_{s}$. This is particularly clear in the case of the singlet at small- $x$ which is substantially smaller in the $N_{f}=3$ scheme due to the missing contribution from the charm and bottom PDFs. In the $N_{f}=4$ case differences are smaller both because we are closer to the heavy quark threshold and because now only bottom is not included into the beta function running and the DGLAP evolution equations.

A similar comparison, but now as a function of scale for fixed $x$ is performed in Fig. 46. The default and FFN PDFs coincide below the matching scale, and become increasingly different as $Q^{2}$ grows. Differences are larger for the gluon, which is coupled by evolution to the singlet, which depends on $N_{f}$ already at leading order.

Several commonly used PDF sets, such as for instance MSTW08 [17] and CT10 [16] use $N_{f}=5$ as a maximum number of flavors. In most cases this makes very little difference at LHC energies, but, again, care must be taken that a scheme in which $N_{f}=6$ above top threshold is sometimes used matrix element calculations such as for example, the NLO Higgs production cross-section of Ref. [93]. To illustrate the size of the effects involved, in Fig. 47 we compare the gluon and the singlet PDFs for the reference NNPDF2.1 set and the FFN set with $N_{f}=5$ as a function of scale at $x=10^{-4}$.

It is beyond the scope of this work to study the phenomenological implications of these FFNS PDFs. However several applications can be envisaged, such as helping to determine the optimal scales in the massless computations when comparing with the massive result, where in each case 

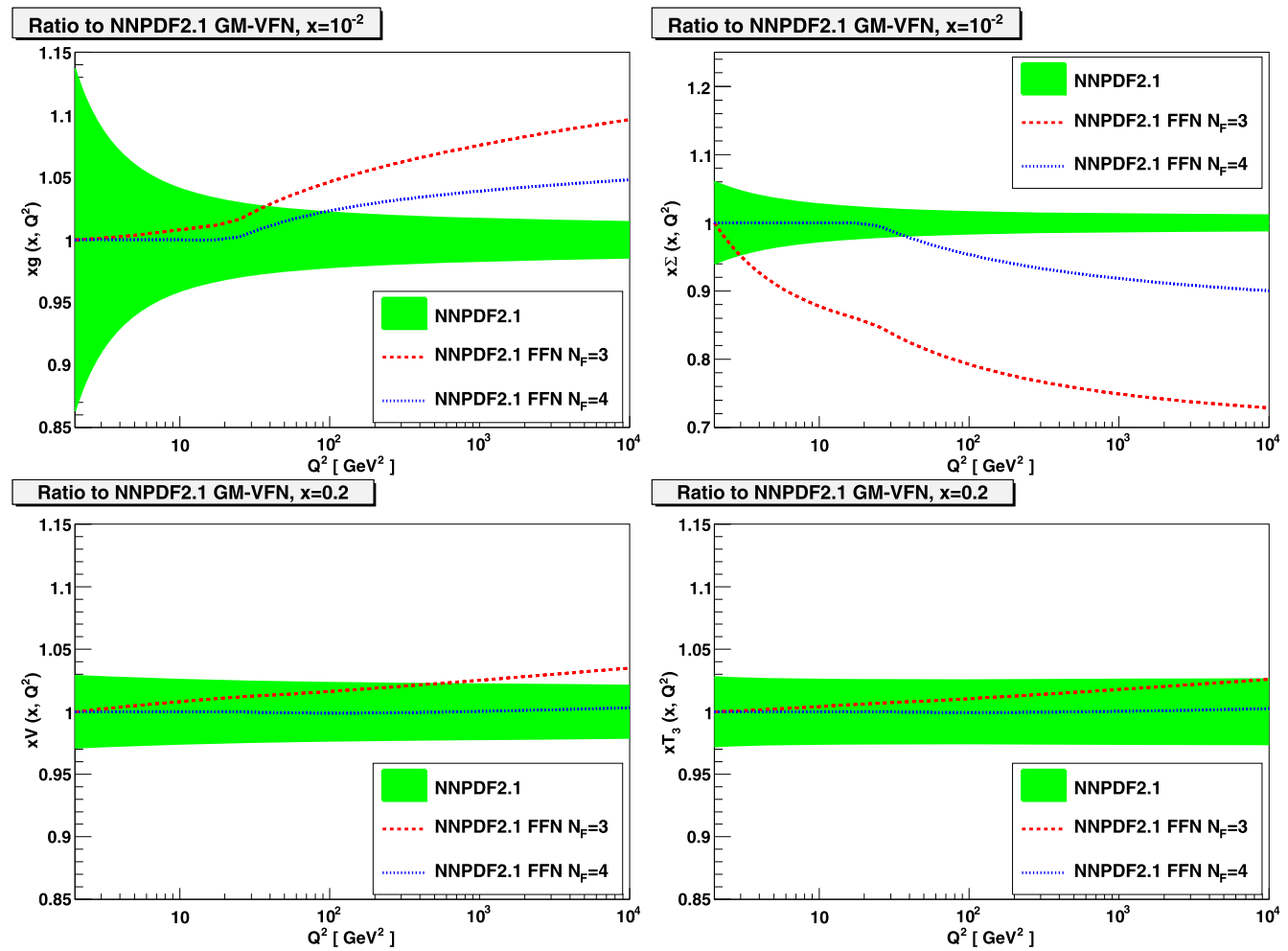

Fig. 46. Comparison between the default NNPDF2.1 set and the $N_{f}=3$ and $N_{f}=4$ FFN sets as a function of scale. Results are shown as ratios to the default set. The gluon and singlet at $x=0.01$ (top) and the triplet and the total valence at $x=0.2$ (bottom) are shown.
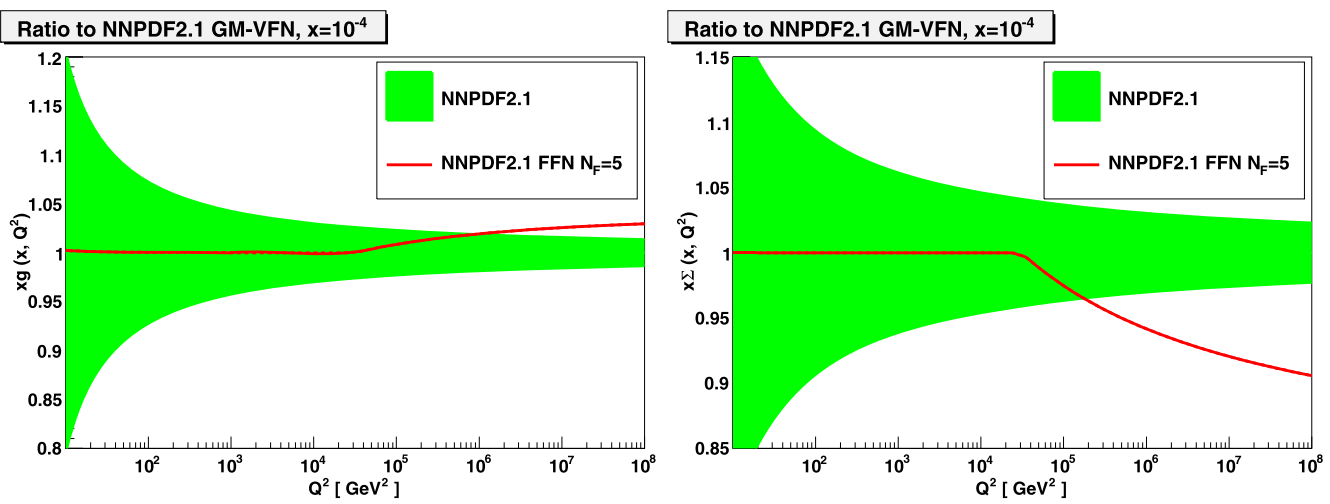

Fig. 47. Comparison between the default NNPDF2.1 set and the $N_{f}=5 \mathrm{FFN}$ set as a function of scale, shown as ratios to the default set. The gluon (left) and singlet (right) are shown. 
the corresponding PDF set in the same scheme has been used. We will discuss these applications in future work.

\section{Conclusions and outlook}

The NNPDF2.1 PDFs presented here fulfill most of the requirements of an ideal [1] NLO parton set: they are based on a global dataset which includes most if not all relevant deep-inelastic and hadronic data, they are free of parametrization bias, they are provided with reliable and statistically meaningful uncertainty estimates, they include NLO contributions without resorting to $K$-factors, they include a consistent treatment of heavy quark mass effects, and they are available for a variety of values of the strong coupling and heavy quark masses, which allows for the estimate of the associated uncertainties. We find that the heavy quark mass effects, while substantial for observables which probe directly the heavy quark distributions, are rather small for more inclusive observables. In particular the benchmark LHC cross-sections (inclusive vector boson, top and Higgs production) change by little more than one standard deviation when going from NNPDF2.0 to NNPDF2.1.

An important missing feature of NNPDF2.1 is a reliable estimate of theoretical uncertainties related to missing higher order corrections (in particular, no study of renormalization and factorization scale dependence is performed). The obvious direction for future improvement is thus the determination of NNLO corrections to PDFs, the inclusion of mass effects at $O\left(\alpha_{s}^{2}\right)$, and the inclusion of resummation corrections at large and small- $x$. We expect all these corrections to be generally rather less than current PDF uncertainties: nonetheless, they need to be computed.

To the extent that the criteria fulfilled by NNPDF2.1 are the dominant ones for accurate phenomenology, NNPDF2.1 is perhaps the most reliable parton set currently available: no other set fulfills all of these criteria. We believe it to be adequate for precise phenomenology at the LHC, at least for the levels of experimental precision to be expected in the near future.

All the NNPDF2.1 PDF sets that have been discussed in this work are available from the NNPDF web site, http://sophia.ecm.ub.es/nnpdf and will be also available through the LHAPDF interface [94].

The PDF sets that have been produced in the present analysis and that will be available in LHAPDF are the following:

- The reference NNPDF2.1 sets, sets of $N_{\text {rep }}=100$ and 1000 replicas: NNPDF21_100.LHgrid and NNPDF21_1000.LHgrid.

- NNPDF2.1 sets of $N_{\text {rep }}=100$ replicas with $\alpha_{s}$ varied from 0.114 to 0.124 with steps of $\delta \alpha_{s}=0.001$ :

NNPDF21_as_0114_100.LHgrid, .., NNPDF21_as_0124_100.LHgrid

- NNPDF2.1 sets with combined PDF $+\alpha_{s}$ uncertainty:

NNPDF21_as_0119_pm_00012_100.LHgrid,

NNPDF21_as_0119_pm_00020_100.LHgrid,

NNPDF21_as_0119_pm_00012_50.LHgrid, and

NNPDF21_as_0119_pm_00020_50.LHgrid

Note than in this case the corresponding NNPDF2.0 sets have also been produced.

- NNPDF2.1 sets based on reduced datasets: DIS only, DIS + DY, DIS + jets:

NNPDF21_dis_100.LHgrid, NNPDF21_dis+dy_100.LHgrid, and NNPDF21_dis+jet_100.LHgrid, as well as NNPDF21_dis_1000.LHgrid

- NNPDF2.1 sets of $N_{\text {rep }}=100$ replicas with varying charm mass: 
NNPDF21_mc_150_100.LHgrid, NNPDF21_mc_160_100.LHgrid, and NNPDF21_mc_170_100.LHgrid.

- NNPDF2.1 sets of $N_{\text {rep }}=100$ replicas with varying bottom mass:

NNPDF21_mb_425_100.LHgrid, NNPDF21_mb_450_100.LHgrid, NNPDF21_mb_500_100.LHgrid, and NNPDF21_mb_525_100.LHgrid.

- NNPDF2.1 sets in the $N_{f}=3, N_{f}=4$ and $N_{f}=5$ FFN schemes:

NNPDF21_FFN_NF3_100.LHgrid, NNPDF21_FFN_NF4_100.LHgrid, and NNPDF21_FFN_NF5_100.LHgrid.

\section{Acknowledgements}

We are especially grateful to G. Watt for providing us the MSTW08 benchmark computations for heavy quark charged current structure functions and for discussions. We are grateful to R. Harlander for providing us with his bbg@ nnlo code. We would like to thank M. Corradi, K. Lipka, B. List and P. Thompson for help with HERA $F_{2}^{c}$ data, S. Glazov for assistance with the HERA $F_{L}$ data, P. Nason for illuminating discussions on heavy quarks, and F. Maltoni, P. Nadolsky and R. Thorne for various discussions. L.D.D. is funded by an STFC Advanced Fellowship and M.U. is supported by the Bundesministerium für Bildung and Forschung (BmBF) of the Federal Republic of Germany (project code 05H09PAE). This work was partly supported by the Spanish MEC FIS2007-60350 grant and by the European network HEPTOOLS under contract MRTN-CT-2006-035505. We would like to acknowledge the use of the computing resources provided by the Black Forest Grid Initiative in Freiburg and by the Edinburgh Compute and Data Facility (ECDF) (http://www.ecdf.ed.ac.uk/). The ECDF is partially supported by the eDIKT initiative (http://www.edikt.org.uk).

\section{Appendix A. Mellin space implementation of neutral current structure functions}

In this appendix we compute the analytic Mellin transform of the $x$-space $\mathcal{O}\left(\alpha_{s}\right)$ heavy quark neutral current coefficient functions and we discuss the implementation and benchmarking of FONLL neutral current structure functions in the FastKernel framework. The corresponding results for charged current structure functions are collected in Appendix B.

The $x$-space gluon $\mathcal{O}\left(\alpha_{s}\right)$ heavy quark coefficient function is given by Eq. (4). Its Mellin transform is defined in the standard way as

$$
C_{2, g}^{\left(n_{l}\right), 1}\left(N, \frac{Q^{2}}{m_{h}^{2}}\right)=\int_{0}^{\left(1+4 m_{h}^{2} / Q^{2}\right)^{-1}} d z z^{N-1} C_{2, g}^{\left(n_{l}\right), 1}\left(z, \frac{Q^{2}}{m_{h}^{2}}\right) .
$$

It is easy to see that the integral (42) can be written in the following way

$$
\begin{aligned}
C_{2, g}^{\left(n_{l}\right), 1}(N, \epsilon)= & T_{R} a^{N} \int_{0}^{1} d t t^{N-1}\left\{\left[1+2 a(2 \epsilon-1) t+2 a^{2}\left(1-6 \epsilon-4 \epsilon^{2}\right) t^{2}\right] \ln \frac{1+v}{1-v}\right. \\
& \left.-\left[1+4 a(\epsilon-2) t-4 a^{2}(\epsilon-2) t^{2}\right] v\right\}
\end{aligned}
$$




$$
\begin{aligned}
= & T_{R} a^{N} \int_{0}^{1} d t t^{N-1}\left\{\left[1+(1-3 a) t-\frac{1}{2}\left(1+4 a-9 a^{2}\right) t^{2}\right] \ln \frac{1+v}{1-v}\right. \\
& \left.-\left[1+(1-9 a) t-a(1-9 a) t^{2}\right] v\right\},
\end{aligned}
$$

where we have defined $a(\epsilon) \equiv(1+4 \epsilon)^{-1}$ to simplify the coefficients. The integrals we need are thus

$$
J_{1}(N) \equiv \int_{0}^{1} d t t^{N-1} \ln \frac{1+v}{1-v}, \quad J_{2}(N) \equiv \int_{0}^{1} d t t^{N-1} v,
$$

since extra powers of $t$ can be accommodated by a shift in $N$ by an integer. Here as usual $v=$ $(1-t)^{1 / 2} /(1-a t)^{1 / 2}$.

The two integrals that we need are related by an integration by parts. To show this, we need

$$
\begin{aligned}
\frac{d}{d t} \ln \frac{1+v}{1-v} & =\frac{d v}{d t} \frac{d}{d v} \ln \frac{1+v}{1-v} \\
& =\left(-\frac{1}{2} \frac{1-a}{(1-t)^{1 / 2}(1-a t)^{3 / 2}}\right)\left(\frac{2(1-a t)}{(1-a) t}\right) \\
& =-\frac{1}{t} \frac{1}{(1-t)^{1 / 2}(1-a t)^{3 / 2}} .
\end{aligned}
$$

Thus

$$
J_{1}(N)=-\frac{1}{N} \int_{0}^{1} d t t^{N} \frac{d}{d t} \ln \frac{1+v}{1-v}=\frac{1}{N} I(N),
$$

where we have defined

$$
I(N) \equiv \int_{0}^{1} d t t^{N-1}(1-t)^{-1 / 2}(1-a t)^{-1 / 2} .
$$

Note that the boundary term in the integration by parts vanishes for all $\operatorname{Re} N>0$, and thus its analytic continuation vanishes for all $N$, so it can be safely ignored. Trivially $J_{2}(N)=$ $I(N)-I(N+1)$. The integral $I(N)$ may be evaluated in the usual way in terms of a standard hypergeometric function:

$$
I(N)=\frac{\Gamma(N) \Gamma\left(\frac{1}{2}\right)}{\Gamma\left(N+\frac{1}{2}\right)} 2 F_{1}\left(\frac{1}{2}, N, N+\frac{1}{2} ; a\right) .
$$

Note that when $a=0$, this reduces to $B\left(N, \frac{1}{2}\right)$, as it should.

We thus get

$$
\begin{aligned}
C_{2, g}^{\left(n_{l}\right), 1}(N, \epsilon)= & T_{R} a^{N}\left\{\left[\frac{1}{N} I(N)+\frac{1-3 a}{N+1} I(N+1)-\frac{1}{2} \frac{1+6 a-9 a^{2}}{N+2} I(N+2)\right]\right. \\
& -[I(N)-I(N+1)+(1-9 a)(I(N+1)-I(N+2))
\end{aligned}
$$




$$
\begin{aligned}
& -a(1-9 a)(I(N+2)-I(N+3))]\} \\
= & T_{R} a^{N}\left\{\left(\frac{1}{N}-1\right) I(N)+\left(\frac{1-3 a}{N+1}+9 a\right) I(N+1)\right. \\
& -\left(\frac{1}{2} \frac{1+4 a-9 a^{2}}{N+2}-(1+a)(1-9 a)\right) I(N+2) \\
& -a(1-9 a) I(N+3)\} .
\end{aligned}
$$

This result is the required ingredient to implement the FONLL-A neutral current structure functions in the FastKernel framework.

A cross-check of the Mellin transform of the massive coefficient function (49) is provided by the fact that its massless limit coincides with the Mellin transform of the $x$-space massive asymptotic $\left(n_{l}, 0\right)$ coefficient function, Eq. (8). To this purposes, we need to expand Eq. (49) near $a=1$. Near $a=1$, i.e. $\epsilon=0$, we need to use the asymptotic expansion

$$
\begin{aligned}
F\left(\frac{1}{2}, N, N+\frac{1}{2} ; a\right)= & \frac{\Gamma\left(N+\frac{1}{2}\right)}{\Gamma\left(\frac{1}{2}\right)^{2} \Gamma(N)^{2}} \sum_{n=0}^{\infty} \frac{\Gamma\left(n+\frac{1}{2}\right) \Gamma(N+n)}{(n !)^{2}} \\
& \times\left[2 \psi(n+1)-\psi\left(n+\frac{1}{2}\right)-\psi(N+n)-\ln (1-a)\right](1-a)^{n},
\end{aligned}
$$

so that

$$
\begin{aligned}
I(N)= & \frac{1}{\Gamma\left(\frac{1}{2}\right) \Gamma(N)} \sum_{n=0}^{\infty} \frac{\Gamma\left(n+\frac{1}{2}\right) \Gamma(N+n)}{(n !)^{2}} \\
& \times\left[2 \psi(n+1)-\psi\left(n+\frac{1}{2}\right)-\psi(N+n)-\ln (1-a)\right](1-a)^{n} .
\end{aligned}
$$

The $n=0$ term then gives the $\ln \epsilon$ collinear divergence, which is subtracted by the massless coefficient function: as $\epsilon \rightarrow 0$

$$
I(N)=-\ln (4 \epsilon)-2 \gamma_{E}-\psi\left(\frac{1}{2}\right)-\psi(N)+O(\epsilon) .
$$

Substituting in Eq. (49) we obtain

$$
C_{g}^{\left(n_{l}\right), 1}(N, \epsilon)=T_{R} \frac{\left[-N^{3}+3 N^{2}-N\left(2+N+N^{2}\right)\left(\ln \epsilon+\gamma_{E}+\psi(N)\right)\right]}{N^{2}(1+N)(2+N)}+\mathcal{O}(\epsilon),
$$

as expected: the coefficient of the singularity is precisely the LO anomalous dimension $\gamma_{q g}^{(0)}(N)$. Therefore we have checked that the massless limit is properly reproduced,

$$
C_{g}^{\left(n_{l}\right), 1}(N, \epsilon)+\mathcal{O}(\epsilon)=C_{g}^{\left(n_{l}, 0\right), 1}(N, \epsilon),
$$

with the massive asymptotic coefficient function given by the Mellin transform of Eq. (8), as desired.

For completeness we also provide the corresponding expressions for the $\mathcal{O}\left(\alpha_{S}\right)$ heavy quark coefficient function for the longitudinal structure function $F_{L, c}$, which is implicitly contained 
in Eq. (49) since $F_{2, c}=F_{T, c}+F_{L, c}$. The $x$-space expression for the longitudinal heavy quark coefficient function is

$$
C_{L, g}^{\left(n_{l}\right), 1}\left(z, \frac{Q^{2}}{m^{2}}\right)=\theta\left(W^{2}-4 m^{2}\right) \times T_{R}\left[-8 \epsilon z^{2} \log \frac{1+v}{1-v}+4 v z(1-z)\right] .
$$

Its Mellin transform can be computed using the integrals discussed above, with the result

$$
\begin{aligned}
& C_{L, g}^{\left(n_{l}\right), 1}\left(N, \frac{Q^{2}}{m^{2}}\right) \\
& =T_{R} a^{N+1}\left[-8 \epsilon a \frac{I(N+2)}{N+2}+4(I(N+1)-I(N+2)(1+a)+I(N+2))\right] .
\end{aligned}
$$

The massless limits of the $x$ - and $N$-space results are straightforwardly computed and checked to be related by Mellin transformation as they ought to.

Now we turn to the implementation and benchmarking of these results in the FastKernel framework. The major improvement in the FastKernel framework as compared to Ref. [10] is the inclusion of heavy quark mass effects in deep-inelastic scattering structure functions, following the FONLL-A general-mass scheme [11]. As discussed in Ref. [10], FastKernel requires to write down all the DIS observables in Mellin space and precomputing all the associated $x$-space Green's functions. Therefore, to extend FastKernel with FONLL structure functions we need to formulate FONLL in Mellin space.

The $x$-space expression for the FONLL-A heavy quark structure functions, Eq. (1), can be easily written down in $N$-space as follows:

$$
\begin{aligned}
F_{2, h}^{\mathrm{FONLL}}\left(N, Q^{2}\right)= & F_{2, h}^{\left(n_{l}\right)}\left(N, Q^{2}\right)-\theta\left(Q^{2}-m^{2}\right)\left(1-\frac{m^{2}}{Q^{2}}\right)^{2} \\
& \times\left[F_{2, h}^{\left(n_{l}, 0\right)}\left(N, Q^{2}\right)-F_{2, h}^{\left(n_{l}+1\right)}\left(N, Q^{2}\right)\right],
\end{aligned}
$$

with the default damping factor as threshold prescription. In order to implement Eq. (56) in the FastKernel framework, we need the Mellin space expressions of the heavy quark coefficient function in the $\left(n_{l}\right),\left(n_{l}, 0\right)$ and $\left(n_{l}+1\right)$ schemes. While the last two are known, the former was not available in a closed form suitable for analytical continuation. The details of the computation have been presented above, and the desired result is Eq. (49).

With all the Mellin space heavy quark coefficient functions available, it becomes possible to implement the FONLL-A heavy quark structure functions, Eq. (56) into the FastKernel framework. To show that the $N$-space implementation has the required accuracy, in Table 14 for $F_{2, c}$ and in Table 15 for $F_{L, c}$, we compare the results for the Les Houches Heavy Quark benchmarks [19] for FONLL-A obtained with the FONLLdis code [11,67] and with the FastKernel framework for various relevant values of $Q^{2}$. The benchmark settings for the PDFs and $\alpha_{s}$ are used for this comparison. What we can see is that the accuracy on the FONLL heavy quark structure functions is essentially always below the percent level, enough for precision phenomenological studies. For completeness we also show the analogous results for the case of the massive scheme results, where similar accuracies are obtained.

\section{Appendix B. Mellin space implementation of charged current structure functions}

In this appendix the analysis of Appendix A is repeated for charged current structure functions. 
Table 14

Results of the benchmark comparison for the $F_{2 c}\left(x, Q^{2}\right)$ structure function in the FONLL-A scheme for the FONLLdis code [11] and for the FastKernel code. Results are provided at the benchmark kinematic points in $x, Q^{2}$. Results for the massive (FFN) scheme are also given for completeness.

\begin{tabular}{|c|c|c|c|c|c|c|}
\hline \multirow{2}{*}{$x$} & \multicolumn{3}{|l|}{ FONLL-A } & \multicolumn{3}{|l|}{ FFN } \\
\hline & FONLLdis & FastKernel & Accuracy & FONLLdis & FastKernel & Accuracy \\
\hline \multicolumn{7}{|c|}{$\overline{Q^{2}=4 \mathrm{GeV}^{2}}$} \\
\hline $10^{-5}$ & 0.1507 & 0.1501 & $0.4 \%$ & 0.1088 & 0.1091 & $0.3 \%$ \\
\hline $10^{-4}$ & 0.0936 & 0.0931 & $0.5 \%$ & 0.0697 & 0.0698 & $0.1 \%$ \\
\hline $10^{-3}$ & 0.0506 & 0.0504 & $0.4 \%$ & 0.0392 & 0.391 & $0.2 \%$ \\
\hline $10^{-2}$ & 0.0174 & 0.0177 & $1.5 \%$ & 0.0136 & 0.0137 & $0.7 \%$ \\
\hline \multicolumn{7}{|c|}{$Q^{2}=10 \mathrm{GeV}^{2}$} \\
\hline $10^{-5}$ & 0.563 & 0.561 & $0.4 \%$ & 0.3598 & 0.3602 & $0.1 \%$ \\
\hline $10^{-4}$ & 0.312 & 0.311 & $0.3 \%$ & 0.2007 & 0.2011 & $0.2 \%$ \\
\hline $10^{-3}$ & 0.1499 & 0.1495 & $0.3 \%$ & 0.0981 & 0.0982 & $0.1 \%$ \\
\hline $10^{-2}$ & 0.05056 & 0.05052 & $0.1 \%$ & 0.0328 & 0.0327 & $0.3 \%$ \\
\hline \multicolumn{7}{|c|}{$Q^{2}=100 \mathrm{GeV}^{2}$} \\
\hline $10^{-5}$ & 2.28636 & 2.28577 & $0.02 \%$ & 1.9779 & 1.9877 & $0.5 \%$ \\
\hline $10^{-4}$ & 1.12186 & 1.12082 & $0.1 \%$ & 0.9161 & 0.9184 & $0.3 \%$ \\
\hline $10^{-3}$ & 0.48008 & 0.47919 & $0.2 \%$ & 0.3644 & 0.3647 & $0.1 \%$ \\
\hline $10^{-2}$ & 0.15207 & 0.15200 & $0.04 \%$ & 0.1037 & 0.1038 & $0.1 \%$ \\
\hline
\end{tabular}

Table 15

Same as Table 14 for the $F_{L c}\left(x, Q^{2}\right)$ structure function.

\begin{tabular}{|c|c|c|c|c|c|c|}
\hline \multirow{2}{*}{$x$} & \multicolumn{3}{|l|}{ FONLL-A } & \multicolumn{3}{|l|}{ FFN } \\
\hline & FONLLdis & FastKernel & Accuracy & FONLLdis & FastKernel & Accuracy \\
\hline \multicolumn{7}{|c|}{$\overline{Q^{2}=4 \mathrm{GeV}^{2}}$} \\
\hline $10^{-5}$ & 0.0130174 & 0.013094 & $0.6 \%$ & 0.009077 & 0.009081 & $0.04 \%$ \\
\hline $10^{-4}$ & 0.008347 & 0.008316 & $0.4 \%$ & 0.005913 & 0.005910 & $0.05 \%$ \\
\hline $10^{-3}$ & 0.004795 & 0.004778 & $0.3 \%$ & 0.003511 & 0.003509 & $0.06 \%$ \\
\hline $10^{-2}$ & 0.001910 & 0.001907 & $0.2 \%$ & 0.001403 & 0.001406 & $0.2 \%$ \\
\hline \multicolumn{7}{|c|}{$Q^{2}=10 \mathrm{GeV}^{2}$} \\
\hline $10^{-5}$ & 0.073235 & 0.073022 & $0.3 \%$ & 0.049856 & 0.049982 & $0.2 \%$ \\
\hline $10^{-4}$ & 0.041392 & 0.041251 & $0.3 \%$ & 0.028402 & 0.028423 & $0.07 \%$ \\
\hline $10^{-3}$ & 0.020754 & 0.020707 & $0.2 \%$ & 0.014463 & 0.014456 & $0.05 \%$ \\
\hline $10^{-2}$ & 0.007616 & 0.007595 & $0.3 \%$ & 0.005350 & 0.005346 & $0.07 \%$ \\
\hline \multicolumn{7}{|c|}{$Q^{2}=100 \mathrm{GeV}^{2}$} \\
\hline $10^{-5}$ & 0.471889 & 0.4729 & $0.2 \%$ & 0.3955 & 0.397855 & $0.6 \%$ \\
\hline $10^{-4}$ & 0.2236 & 0.2235 & $0.1 \%$ & 0.18656 & 0.186914 & $0.2 \%$ \\
\hline $10^{-3}$ & 0.0920 & 0.09188 & $0.1 \%$ & 0.0765 & 0.076393 & $0.1 \%$ \\
\hline $10^{-2}$ & 0.027822 & 0.02782 & $0.1 \%$ & 0.023079 & 0.023100 & $0.1 \%$ \\
\hline
\end{tabular}

The $F_{2}^{c}$ charm structure functions in charged current DIS is given by Eq. (9). The expression for structure functions in neutrino-induced charged current scattering in the FFN scheme is 


$$
\begin{aligned}
\mathcal{F}_{i}^{c}\left(x, Q^{2}\right)= & \frac{1}{2} s^{\prime}\left(\xi, \mu^{2}\right) \\
& +\frac{1}{2} \frac{\alpha_{s}\left(\mu^{2}\right)}{2 \pi}\left\{\int _ { \xi } ^ { 1 } \frac { d \xi ^ { \prime } } { \xi ^ { \prime } } \left[C_{i, q}^{\left(n_{l}\right)}\left(\xi^{\prime}, \mu^{2}, \lambda\right) s^{\prime}\left(\frac{\xi}{\xi^{\prime}}, \mu^{2}\right)\right.\right. \\
& \left.\left.+C_{i, g}^{\left(n_{l}\right)}\left(\xi^{\prime}, \mu^{2}, \lambda\right) g\left(\frac{\xi}{\xi^{\prime}}, \mu^{2}\right)\right]\right\},
\end{aligned}
$$

with $i=1,2$, 3. In Eq. (57) have used the following definitions:

$$
\begin{aligned}
& s^{\prime}=2\left|V_{\mathrm{cs}}\right|^{2} s+2\left|V_{\mathrm{cd}}\right|^{2}[f d+(1-f) u] ; \quad f=\frac{N_{p}}{N_{p}+N_{n}} \\
& \xi=x\left(1+\frac{m_{c}^{2}}{Q^{2}}\right) ; \quad \lambda=\frac{Q^{2}}{Q^{2}+m_{c}^{2}} .
\end{aligned}
$$

The explicit $x$-space expressions of the $\mathcal{O}\left(\alpha_{s}\right)$ contributions $C_{i, q(g)}^{\left(n_{l}\right)}$ to the coefficient functions are given in Refs. $[62,63]$. The standard structure functions are related to those defined in Eq. (57) through

$$
F_{1}^{c} \equiv \mathcal{F}_{1}^{c} ; \quad F_{2}^{c} \equiv 2 \xi \mathcal{F}_{2}^{c}=x \frac{2}{\lambda} \mathcal{F}_{2}^{c} ; \quad F_{3}^{c} \equiv 2 \mathcal{F}_{3}^{c},
$$

so that

$$
F_{L}^{c} \equiv F_{2}^{c}-2 x F_{1}^{c}=2 \xi\left(\mathcal{F}_{2}^{c}-\lambda \mathcal{F}_{1}^{c}\right) .
$$

Before Mellin-transforming the $x$-space quark coefficient functions of Refs. [62,63] we rewrite them all in the form

$$
C_{i, q}^{\left(n_{l}\right)}(x)=K \delta(1-x)+f(x)+[g(x)]_{+},
$$

where $K$ is a constant and $f(x)$ is regular function in $x \in[0,1]$ (so in general $g(x)$ is not regular in $x=1$ ). We get

$$
\begin{aligned}
C_{1, q}^{\left(n_{l}\right)}(z)= & -C_{F}\left(4+\frac{1}{2 \lambda}+\frac{\pi^{2}}{3}+\frac{1+3 \lambda}{2 \lambda} K_{A}\right) \delta(1-z) \\
& +C_{F}\left[-\frac{\left(1+z^{2}\right) \ln z}{1-z}-2(1+z) \ln (1-z)+(1+z) \ln (1-\lambda z)+(3-z)\right. \\
& \left.+\frac{z-z^{2}}{1-\lambda z}\right] \\
& +C_{F}\left[4 \frac{\ln (1-z)}{1-z}-2 \frac{\ln (1-\lambda z)}{1-z}-2 \frac{1}{1-z}+\frac{1}{2} \frac{1-z}{(1-\lambda z)^{2}}-\frac{1+z^{2}}{1-z} \ln \lambda\right]_{+} ; \\
C_{2, q}^{\left(n_{l}\right)}(z)= & -C_{F}\left(4+\frac{1}{2 \lambda}+\frac{\pi^{2}}{3}+\frac{1+\lambda}{2 \lambda} K_{A}\right) \delta(1-z) \\
& +C_{F}\left[-\frac{\left(1+z^{2}\right) \ln z}{1-z}-2(1+z) \ln (1-z)+(1+z) \ln (1-\lambda z)\right.
\end{aligned}
$$




$$
\begin{aligned}
& \left.+\left(2 z+2-\frac{2}{z}\right)+\frac{\frac{2}{z}-1-z}{1-\lambda z}\right] \\
& +C_{F}\left[4 \frac{\ln (1-z)}{1-z}-2 \frac{\ln (1-\lambda z)}{1-z}-2 \frac{1}{1-z}+\frac{1}{2} \frac{1-z}{(1-\lambda z)^{2}}-\frac{1+z^{2}}{1-z} \ln \lambda\right]_{+} ; \\
C_{3, q}^{\left(n_{l}\right)}(z)= & -C_{F}\left(4+\frac{1}{2 \lambda}+\frac{\pi^{2}}{3}+\frac{1+3 \lambda}{2 \lambda} K_{A}\right) \delta(1-z) \\
& +C_{F}\left[-\frac{\left(1+z^{2}\right) \ln z}{1-z}-2(1+z) \ln (1-z)\right. \\
& \left.+(1+z) \ln (1-\lambda z)+(1+z)+\frac{1-z}{1-\lambda z}\right] \\
& +C_{F}\left[4 \frac{\ln (1-z)}{1-z}-2 \frac{\ln (1-\lambda z)}{1-z}-2 \frac{1}{1-z}+\frac{1}{2} \frac{1-z}{(1-\lambda z)^{2}}-\frac{1+z^{2}}{1-z} \ln \lambda\right]_{+}
\end{aligned}
$$

with $K_{A}=(1-\lambda) \ln (1-\lambda) / \lambda$.

The gluon coefficient functions do not need any further work and are given by

$$
\begin{aligned}
C_{1, g}^{\left(n_{l}\right)}(z)= & T_{f}\left(2 z^{2}-2 z+1\right)\{2 \ln (1-z)-2 \ln z-\ln [\lambda(1-\lambda)]\} \\
& +[4-4(1-\lambda)] z(1-z)+(1-\lambda) \frac{z}{1-\lambda z} \\
& +2(1-\lambda)\left[z \ln \frac{1-\lambda z}{(1-\lambda) z}-2 \lambda z^{2} \ln \frac{1-\lambda z}{(1-\lambda) z}\right]-1 ; \\
C_{2, g}^{\left(n_{l}\right)}(z)= & T_{f}\left(2 z^{2}-2 z+1\right)\{2 \ln (1-z)-2 \ln z-\ln [\lambda(1-\lambda)]\} \\
& +\left[8-18(1-\lambda)+12(1-\lambda)^{2}\right] z(1-z)+(1-\lambda) \frac{1}{1-\lambda z} \\
& +6 \lambda(1-\lambda)\left[z \ln \frac{1-\lambda z}{(1-\lambda) z}-2 \lambda z^{2} \ln \frac{1-\lambda z}{(1-\lambda) z}\right]-1 ; \\
C_{3, g}^{\left(n_{l}\right)}(z)= & T_{f}\left(2 z^{2}-2 z+1\right)\left\{2 \ln (1-z)-2 \ln (1-\lambda z)+\ln \left(\frac{1-\lambda}{\lambda}\right)\right\} \\
& +2(1-\lambda) z(1-z)+2(1-\lambda)\left[(1+\lambda) z^{2} \ln \frac{1-\lambda z}{(1-\lambda) z}-z \ln \frac{1-\lambda z}{(1-\lambda) z}\right] .
\end{aligned}
$$

In order to transform to the $N$-space the above $x$-space expressions, in Tables 16 and 17 we tabulate the Mellin transforms of all terms involved. In these tables we use the analytic continuation of the harmonic sum

$$
S_{l} \equiv S_{l}(N)=\sum_{k=1}^{N} \frac{1}{k^{l}}=\zeta(l)-\frac{(-1)^{l}}{(l-1) !} \psi^{(l-1)}(N+1),
$$

where $\zeta(l)$ is the Riemann $\zeta$-function, with $\zeta(1)=\gamma_{E M}, \psi((l-1))$ is the polygamma, and ${ }_{2} F_{1}(a, b, c ; N)$ is the Gauss hypergeometric function.

As an example of use of Tables 16-17, we present here the complete $N$-space quark and gluon coefficient functions for $F_{2}^{c}$ 
Table 16

Mellin transforms of the terms involved in the NLO charged current quark coefficient functions.

\begin{tabular}{ll}
\hline$f(z)$ & $\mathbf{M}[f](N)$ \\
\hline$\delta(1-z)$ & 1 \\
$z^{l}$ & $\frac{1}{N+l}$ \\
$\frac{\left(1+z^{2}\right) \ln z}{1-z}$ & $2\left(S_{2}-\zeta_{2}\right)-\frac{1}{N^{2}}+\frac{1}{(N+1)^{2}}$ \\
$(1+z) \ln (1-z)$ & $-\frac{S_{1}}{N}-\frac{S_{1}}{N+1}-\frac{1}{(N+1)^{2}}$ \\
$(1+z) \ln (1-\lambda z)$ & $\lambda \frac{2 F_{1}(1, N+1, N+2 ; \lambda)}{N(N+1)}+\frac{\ln (1-\lambda)}{N}+\lambda \frac{2 F_{1}(1, N+2, N+3 ; \lambda)}{(N+1)(N+2)}+\frac{\ln (1-\lambda)}{N+1}$ \\
$\frac{z-z^{2}}{1-\lambda z}$ & $\frac{2 F_{1}(1, N+1, N+2, \lambda)}{N+1}-\frac{2 F_{1}(1, N+2, N+3, \lambda)}{N+2}$ \\
$\frac{2}{z}-1-z$ \\
$1-\lambda z$ & $2 \frac{2 F_{1}(1, N-1, N, \lambda)}{N-1}-\frac{2 F_{1}(1, N, N+1, \lambda)}{N}-\frac{2 F_{1}(1, N+1, N+2, \lambda)}{N+1}$ \\
$\frac{1-z}{1-\lambda z}$ & $\frac{{ }_{2} F_{1}(1, N, N+1, \lambda)}{N}-\frac{{ }_{2} F_{1}(1, N+1, N+2, \lambda)}{N+1}$ \\
{$\left[\frac{\ln (1-z)}{1-z}\right]_{+}$} & $\frac{1}{2}\left(S_{1}^{2}+S_{2}-2 \frac{S_{1}}{N}\right)$ \\
{$\left[\frac{\ln (1-\lambda z)}{1-z}\right]_{+}$} & $J_{\lambda}(N)=\sum_{k=1}^{\infty} \frac{\lambda^{k}}{k}\left[S_{1}(N+k)-S_{1}(k)-\frac{1}{N+k}\right]$ \\
{$\left[\frac{1}{1-z}\right]_{+}$} & $\frac{1}{N}-S_{1}$ \\
{$\left[\frac{1-z}{(1-\lambda z)^{2}}\right]_{+}$} & $\frac{2 F_{1}(2, N, N+2, \lambda)}{N(N+1)}+\frac{\lambda+\ln (1-\lambda)}{\lambda^{2}}$ \\
{$\left[\frac{1+z^{2}}{1-z}\right]_{+}$} & $\frac{1}{N}-\frac{1}{N+1}-2 S_{1}+\frac{3}{2}$ \\
\hline
\end{tabular}

Table 17

Mellin transforms of the terms involved in the NLO charged current gluon coefficient functions.

\begin{tabular}{ll}
\hline$f(z)$ & $\mathbf{M}[f](N)$ \\
\hline$\left[z^{2}+(1-z)^{2}\right]\left\{2 \ln \left(\frac{1-z}{z}\right)-\ln [\lambda(1-\lambda)]\right\}$ & $\frac{4-2 N(N-3)-N\left(N^{2}+N+2\right)\left\{2 S_{1}+\ln [\lambda(1-\lambda)]\right\}}{N^{2}(N+1)(N+2)}$ \\
{$\left[z^{2}+(1-z)^{2}\right]\left\{2 \ln \left(\frac{1-z}{1-\lambda z}\right)+\ln \left(\frac{1-\lambda}{\lambda}\right)\right\}$} & $-\frac{2}{\lambda}\left(\frac{\lambda^{2}}{N}-\frac{2 \lambda}{N+1}+\frac{2}{N+2}\right) \frac{2 F_{1}(1, N+1, N+2, \lambda)}{N+1}-\frac{4(\lambda-1)}{\lambda(N+1)(N+2)}$ \\
$z \ln \frac{1-\lambda z}{(1-\lambda) z}$ & $-\frac{\left(N^{2}+N+2\right)\left\{2 S_{1}-\ln \left(\frac{1-\lambda}{\lambda}\right)\right\}}{N(N+1)(N+2)}$ \\
$z^{2} \ln \frac{1-\lambda z}{(1-\lambda) z}$ & $\frac{2 F_{1}(1, N+1, N+2 ; \lambda)}{(N+1)^{2}}$ \\
$\frac{1}{1-\lambda z}$ & $\frac{{ }_{2} F_{1}(1, N+1, N+2 ; \lambda)-1}{\lambda(N+1)(N+2)}$ \\
$\frac{z}{1-\lambda z}$ & $\frac{{ }_{2} F_{1}(1, N, N+1 ; \lambda)}{N}$ \\
& $\frac{2 F_{1}(1, N+1, N+2 ; \lambda)}{N+1}$ \\
\hline
\end{tabular}

$$
\begin{aligned}
C_{2, q}^{\left(n_{l}\right)}(N)= & C_{F}\left[-\left(4+\frac{1}{2 \lambda}+\frac{\pi^{2}}{3}+\frac{1+\lambda}{2 \lambda} K_{A}\right)-2\left(S_{2}-\zeta_{2}\right)+\frac{1}{N^{2}}\right. \\
& -\frac{1}{(N+1)^{2}}+2\left(\frac{S_{1}}{N}+\frac{S_{1}}{N+1}+\frac{1}{(N+1)^{2}}\right)+\lambda \frac{2 F_{1}(1, N+1, N+2 ; \lambda)}{N(N+1)} \\
& +\frac{\ln (1-\lambda)}{N}+\lambda \frac{{ }_{2} F_{1}(1, N+2, N+3 ; \lambda)}{(N+1)(N+2)}+\frac{\ln (1-\lambda)}{N+1}+\frac{2}{N+1}+\frac{2}{N} \\
& -\frac{2}{N-1}+2 \frac{{ }_{2} F_{1}(1, N-1, N, \lambda)}{N-1}-\frac{{ }_{2} F_{1}(1, N, N+1, \lambda)}{N} \\
& -\frac{{ }_{2} F_{1}(1, N+1, N+2, \lambda)}{N+1}+2\left(S_{1}^{2}+S_{2}-2 \frac{S_{1}}{N}\right)-2 J_{\lambda}(N)-2\left(\frac{1}{N}-S_{1}\right)
\end{aligned}
$$




$$
\begin{aligned}
& +\frac{1}{2}\left(\frac{2 F_{1}(2, N, N+2, \lambda)}{N(N+1)}+\frac{\lambda+\ln (1-\lambda)}{\lambda^{2}}\right) \\
& \left.-\left(\frac{1}{N}-\frac{1}{N+1}-2 S_{1}+\frac{3}{2}\right) \ln \lambda\right] ; \\
C_{2, g}^{\left(n_{l}\right)}(N)= & T_{f} \frac{4-2 N(N-3)-N\left(N^{2}+N+2\right)\left\{S_{1}+\ln [\lambda(1-\lambda)]\right\}}{2 N^{2}(N+1)(N+2)} \\
& +\frac{8-18(1-\lambda)+12(1-\lambda)^{2}}{(N+1)(N+2)}+\frac{(1-\lambda)_{2} F_{1}(1, N, N+1 ; \lambda)}{N} \\
& +6 \lambda(1-\lambda)\left[\frac{2 F_{1}(1, N+1, N+2 ; \lambda)}{(N+1)^{2}}\right. \\
& \left.-\frac{2{ }_{2} F_{1}(1, N+1, N+2 ; \lambda)-1}{(N+1)(N+2)}\right]-\frac{1}{N} .
\end{aligned}
$$

As a cross-check of the Mellin space results, it is possible to compute the asymptotic limit $\lambda \rightarrow 1$ of these expressions. We need the asymptotic expansion of the hypergeometric functions, Eq. (50), up to $\mathcal{O}(\lambda-1)$ terms. In particular,

$$
\begin{aligned}
& { }_{2} F_{1}(1, N+1, N+2 ; \lambda)=-(1+N)\left(\ln (1-\lambda)+\gamma_{E}+\psi^{(0)}(N+1)\right)+\mathcal{O}((\lambda-1)), \\
& { }_{2} F_{1}(2, N, N+2 ; \lambda)=-N(1+N)\left(\ln (1-\lambda)+\gamma_{E}+\psi^{(0)}(N)\right)+\mathcal{O}((\lambda-1)) .
\end{aligned}
$$

Substituting in Eq. (67), one can see that all collinear heavy quark logarithms and that the massless limit of the massive charged current heavy quark coefficient functions reduces to the usual ZM-VFN result, as we know from $x$-space.

Now we turn to discuss the implementation and benchmarking of the above results into the FastKernel framework. Analogously to the neutral current sector, the FONLL-A charged current structure functions in Mellin space can be written as

$$
\begin{aligned}
& F_{i, h}^{\mathrm{CC}, \mathrm{FONLL}}\left(N, Q^{2}\right) \\
& \quad=F_{i, h}^{\mathrm{CC}\left(n_{l}\right)}\left(N, Q^{2}\right) \\
& \quad-\theta\left(Q^{2}-m^{2}\right)\left(1-\frac{m^{2}}{Q^{2}}\right)^{2}\left[F_{i, h}^{\mathrm{CC}\left(n_{l}, 0\right)}\left(N, Q^{2}\right)-F_{2, h}^{\mathrm{CC}\left(n_{l}+1\right)}\left(N, Q^{2}\right)\right],
\end{aligned}
$$

with $i=1,2,3$. The Mellin space expressions of the massive heavy quark coefficient functions have been computed above, and the other ingredients of Eq. (71) are their massless limits and the standard Mellin transform of the ZM-VFN coefficient functions.

With these results, we have implemented the FONLL-A charged current structure functions (71) into the FastKernel framework. As it has been done in the case of neutral current observables, here we benchmark the accuracy of this FONLL scheme implementation. We use again the same settings of the Les Houches heavy quarks benchmark study. The benchmarking of the FONLL-A $\mathrm{CC}$ structure function implementation in FastKernel is performed for the charm production crosssection in neutrino induced DIS, defined by Eq. (33), that combines all three charged current structure functions. We have checked that the comparison of individual structure functions has a similar level of accuracy.

Results for the benchmark comparison are shown in Table 18. As discussed above, the FONLL-A calculation of charged current structure functions has been implemented in an $x$-space 
Table 18

Results of the benchmark comparison for the dimuon charm production cross-section (33), in the FONLL-A scheme for the FONLLdisCC charged current code and for the FastKernel framework. Results are provided at the benchmark kinematic points in $x, Q^{2}$. Results for the massive (FFN) scheme are also given for completeness. The inelasticity variable in the dimuon cross-section for this benchmark table has been taken to be $y=0.5$. The Les Houches Heavy Quark benchmark settings [19] have been used for the comparison.

\begin{tabular}{|c|c|c|c|c|c|c|}
\hline \multirow{2}{*}{$x$} & \multicolumn{3}{|l|}{ FONLL-A } & \multicolumn{3}{|l|}{ FFN } \\
\hline & FONLLdisCC & FastKernel & Accuracy & FONLLdisCC & FastKernel & Accuracy \\
\hline \multicolumn{7}{|c|}{$Q^{2}=4 \mathrm{GeV}^{2}$} \\
\hline $10^{-5}$ & 163.14 & 164.06 & $0.6 \%$ & 158.70 & 158.15 & $0.3 \%$ \\
\hline $10^{-4}$ & 109.48 & 109.55 & $0.1 \%$ & 106.81 & 106.64 & $0.2 \%$ \\
\hline $10^{-3}$ & 69.24 & 69.35 & $0.2 \%$ & 67.86 & 67.88 & $0.1 \%$ \\
\hline $10^{-2}$ & 37.75 & 37.87 & $0.3 \%$ & 37.27 & 37.30 & $0.1 \%$ \\
\hline $10^{-1}$ & 13.56 & 13.57 & $0.1 \%$ & 13.53 & 13.51 & $0.1 \%$ \\
\hline \multicolumn{7}{|c|}{$Q^{2}=10 \mathrm{GeV}^{2}$} \\
\hline $10^{-5}$ & 279.31 & 278.71 & $0.2 \%$ & 261.49 & 261.55 & $0.02 \%$ \\
\hline $10^{-4}$ & 167.02 & 166.85 & $0.1 \%$ & 157.27 & 157.11 & $0.1 \%$ \\
\hline $10^{-3}$ & 92.90 & 92.87 & $0.03 \%$ & 88.33 & 88.12 & $0.2 \%$ \\
\hline $10^{-2}$ & 44.92 & 44.93 & $0.02 \%$ & 43.36 & 43.23 & $0.3 \%$ \\
\hline $10^{-1}$ & 14.50 & 14.48 & $0.1 \%$ & 14.26 & 14.28 & $0.1 \%$ \\
\hline \multicolumn{7}{|c|}{$Q^{2}=100 \mathrm{GeV}^{2}$} \\
\hline $10^{-5}$ & 674.55 & 674.53 & $0.02 \%$ & 651.21 & 645.94 & $0.1 \%$ \\
\hline $10^{-4}$ & 345.73 & 345.81 & $0.02 \%$ & 331.17 & 329.14 & $0.5 \%$ \\
\hline $10^{-3}$ & 161.70 & 161.78 & $0.05 \%$ & 153.94 & 152.36 & $0.1 \%$ \\
\hline $10^{-2}$ & 64.20 & 64.26 & $0.1 \%$ & 61.11 & 61.06 & $0.1 \%$ \\
\hline $10^{-1}$ & 15.79 & 15.83 & $0.2 \%$ & 15.33 & 15.42 & $0.1 \%$ \\
\hline
\end{tabular}

code, FONLLdisCC, that we will use for the benchmarking with the FastKernel implementation. Results are shown for various values of $Q^{2}$ relevant for the analysis of experimental data. The accuracy is similar to the one achieved for neutral current structure functions (see Tables 14-15), at the per mille level, suitable for precision PDF determinations.

\section{References}

[1] S. Forte, arXiv:1011.5247, 2010.

[2] D. Stump, et al., JHEP 0310 (2003) 046, hep-ph/0303013.

[3] W.K. Tung, et al., JHEP 0702 (2007) 053, hep-ph/0611254.

[4] A. Guffanti, J. Rojo, arXiv:1008.4671, 2010.

[5] S. Forte, et al., JHEP 0205 (2002) 062, hep-ph/0204232.

[6] NNPDF Collaboration, L. Del Debbio, et al., JHEP 0503 (2005) 080, hep-ph/0501067.

[7] NNPDF Collaboration, L. Del Debbio, et al., JHEP 0703 (2007) 039, hep-ph/0701127.

[8] NNPDF Collaboration, R.D. Ball, et al., Nucl. Phys. B 809 (2009) 1, arXiv:0808.1231.

[9] NNPDF Collaboration, R.D. Ball, et al., Nucl. Phys. B 823 (2009) 195, arXiv:0906.1958.

[10] NNPDF Collaboration, R.D. Ball, et al., Nucl. Phys. B 838 (2010) 136, arXiv:1002.4407.

[11] S. Forte, et al., Nucl. Phys. B 834 (2010) 116, arXiv:1001.2312.

[12] M. Cacciari, M. Greco, P. Nason, JHEP 9805 (1998) 007, hep-ph/9803400.

[13] M. Kramer, F.I. Olness, D.E. Soper, Phys. Rev. D 62 (2000) 096007, hep-ph/0003035.

[14] M.A.G. Aivazis, et al., Phys. Rev. D 50 (1994) 3102, hep-ph/9312319.

[15] P.M. Nadolsky, et al., Phys. Rev. D 78 (2008) 013004, arXiv:0802.0007.

[16] H.L. Lai, et al., Phys. Rev. D 82 (2010) 074024, arXiv:1007.2241.

[17] A.D. Martin, et al., Eur. Phys. J. C 63 (2009) 189, arXiv:0901.0002. 
[18] R.S. Thorne, Phys. Rev. D 73 (2006) 054019, hep-ph/0601245.

[19] J. Rojo, et al., in: J.R. Andersen et al., The SM and NLO Multileg Working Group: Summary Report (Ch. 22) arXiv:1003.1241, 2010.

[20] R.D. Ball, et al., in: J.R. Andersen et al., The SM and NLO Multileg Working Group: Summary Report (Ch. 21) arXiv:1003.1241, 2010.

[21] F. Demartin, et al., Phys. Rev. D 82 (2010) 014002, arXiv:1004.0962.

[22] F. Caola, S. Forte, J. Rojo, Nucl. Phys. A 854 (2011) 32, arXiv:1007.5405, 2010.

[23] F. Caola, S. Forte, J. Rojo, Phys. Lett. B 686 (2010) 127, arXiv:0910.3143.

[24] S. Alekhin, et al., Phys. Rev. D 81 (2010) 014032, arXiv:0908.2766.

[25] H1 and ZEUS Collaborations, F.D. Aaron, et al., arXiv:0911.0884, 2009.

[26] NNPDF Collaboration, R.D. Ball, et al., arXiv:1012.0836, 2010.

[27] ZEUS Collaboration, J. Breitweg, et al., Eur. Phys. J. C 12 (2000) 35, hep-ex/9908012.

[28] ZEUS Collaboration, S. Chekanov, et al., Phys. Rev. D 69 (2004) 012004, hep-ex/0308068.

[29] ZEUS Collaboration, S. Chekanov, et al., Eur. Phys. J. C 63 (2009) 171, arXiv:0812.3775.

[30] ZEUS Collaboration, S. Chekanov, et al., Eur. Phys. J. C 65 (2010) 65, arXiv:0904.3487.

[31] H1 Collaboration, C. Adloff, et al., Phys. Lett. B 528 (2002) 199, hep-ex/0108039.

[32] H1 Collaboration, F.D. Aaron, et al., Phys. Lett. B 686 (2010) 91, arXiv:0911.3989.

[33] H1 Collaboration, F.D. Aaron, et al., Eur. Phys. J. C 65 (2010) 89, arXiv:0907.2643.

[34] New Muon Collaboration, M. Arneodo, et al., Nucl. Phys. B 487 (1997) 3, hep-ex/9611022.

[35] New Muon Collaboration, M. Arneodo, et al., Nucl. Phys. B 483 (1997) 3, hep-ph/9610231.

[36] L.W. Whitlow, et al., Phys. Lett. B 282 (1992) 475.

[37] BCDMS Collaboration, A.C. Benvenuti, et al., Phys. Lett. B 223 (1989) 485.

[38] BCDMS Collaboration, A.C. Benvenuti, et al., Phys. Lett. B 237 (1990) 592.

[39] CHORUS Collaboration, G. Onengut, et al., Phys. Lett. B 632 (2006) 65.

[40] H1 Collaboration, F.D. Aaron, et al., Phys. Lett. B 665 (2008) 139, arXiv:0805.2809.

[41] NuTeV Collaboration, M. Goncharov, et al., Phys. Rev. D 64 (2001) 112006, hep-ex/0102049.

[42] D.A. Mason, FERMILAB-THESIS-2006-01.

[43] ZEUS Collaboration, S. Chekanov, et al., Eur. Phys. J. C 62 (2009) 625, arXiv:0901.2385.

[44] ZEUS Collaboration, S. Chekanov, et al., Eur. Phys. J. C 61 (2009) 223, arXiv:0812.4620.

[45] G. Moreno, et al., Phys. Rev. D 43 (1991) 2815.

[46] NuSea Collaboration, J.C. Webb, et al., hep-ex/0302019, 2003.

[47] J.C. Webb, hep-ex/0301031, 2003.

[48] FNAL E866/NuSea Collaboration, R.S. Towell, et al., Phys. Rev. D 64 (2001) 052002, hep-ex/0103030.

[49] CDF Collaboration, T. Aaltonen, et al., Phys. Rev. Lett. 102 (2009) 181801, arXiv:0901.2169.

[50] D0 Collaboration, V.M. Abazov, et al., Phys. Rev. D 76 (2007) 012003, hep-ex/0702025.

[51] CDF Collaboration, T. Aaltonen, et al., arXiv:0908.3914, 2009.

[52] CDF - Run II Collaboration, A. Abulencia, et al., Phys. Rev. D 75 (2007) 092006, hep-ex/0701051.

[53] D0 Collaboration, V.M. Abazov, et al., Phys. Rev. Lett. 101 (2008) 062001, arXiv:0802.2400.

[54] G. Altarelli, S. Forte, G. Ridolfi, Nucl. Phys. B 534 (1998) 277, hep-ph/9806345.

[55] P.M. Nadolsky, W.K. Tung, Phys. Rev. D 79 (2009) 113014, arXiv:0903.2667.

[56] E. Witten, Nucl. Phys. B 104 (1976) 445.

[57] M.A. Shifman, A.I. Vainshtein, V.I. Zakharov, Nucl. Phys. B 136 (1978) 157.

[58] J.P. Leveille, T.J. Weiler, Nucl. Phys. B 147 (1979) 147.

[59] S.I. Alekhin, J. Blumlein, Phys. Lett. B 594 (2004) 299, hep-ph/0404034.

[60] M. Buza, W.L. van Neerven, Nucl. Phys. B 500 (1997) 301, hep-ph/9702242.

[61] G. Corcella, A.D. Mitov, Nucl. Phys. B 676 (2004) 346, hep-ph/0308105.

[62] M. Gluck, S. Kretzer, E. Reya, Phys. Lett. B 380 (1996) 171, hep-ph/9603304.

[63] T. Gottschalk, Phys. Rev. D 23 (1981) 56.

[64] E.B. Zijlstra, W.L. van Neerven, Nucl. Phys. B 383 (1992) 525.

[65] Particle Data Group, K. Nakamura, J. Phys. G 37 (2010) 075021.

[66] D. Mason, et al., Phys. Rev. Lett. 99 (2007) 192001.

[67] J. Rojo, et al., Fonlldis, http://wwwteor.mi.infn.it/ rojo/fonlldis.html, 2010.

[68] G. Watt, Private communication, 2010.

[69] NNPDF Collaboration, R.D. Ball, et al., JHEP 1005 (2010) 075, arXiv:0912.2276.

[70] ATLAS Collaboration, arXiv:1010.2130, 2010.

[71] CMS Collaboration, arXiv:1010.5994, 2010. 
[72] ATLAS Collaboration, arXiv:1009.5908, 2010.

[73] ATLAS Collaboration, arXiv:1012.1792, 2010.

[74] J. Campbell, R.K. Ellis, Phys. Rev. D 65 (2002) 113007, hep-ph/0202176.

[75] MCFM Collaboration, http://mcfm.fnal.gov.

[76] A.D. Martin, et al., Eur. Phys. J. C 64 (2009) 653, arXiv:0905.3531.

[77] H.L. Lai, et al., Phys. Rev. D 82 (2010) 054021, arXiv:1004.4624.

[78] J.M. Campbell, J.W. Huston, W.J. Stirling, Rep. Prog. Phys. 70 (2007) 89, hep-ph/0611148.

[79] M. Botje, et al., arXiv:1101.0538, 2011.

[80] S. Davidson, et al., JHEP 0202 (2002) 037, hep-ph/0112302.

[81] ZEUS Collaboration, S. Chekanov, et al., Phys. Lett. B 682 (2009) 8, arXiv:0904.1092.

[82] H1 Collaboration, arXiv:1012.4355, 2010.

[83] J. Rojo, Talk at the PDF4LHC workshop, DESY, Hamburg, http://indico.cern.ch/materialDisplay.py?contribId= 7\&sessionId=2\&materialId=slides\&confId=103872, 2010.

[84] A.D. Martin, et al., Eur. Phys. J. C 70 (2010) 51, arXiv:1007.2624.

[85] S. Alekhin, S. Moch, arXiv:1011.5790, 2010.

[86] J. Pumplin, H.L. Lai, W.K. Tung, Phys. Rev. D 75 (2007) 054029, hep-ph/0701220.

[87] S.J. Brodsky, et al., Phys. Lett. B 93 (1980) 451.

[88] R.V. Harlander, W.B. Kilgore, Phys. Rev. D 68 (2003) 013001, hep-ph/0304035.

[89] J.M. Campbell, et al., Phys. Rev. Lett. 102 (2009) 182003, arXiv:0903.0005.

[90] F. Maltoni, Z. Sullivan, S. Willenbrock, Phys. Rev. D 67 (2003) 093005, hep-ph/0301033.

[91] B.W. Harris, J. Smith, Nucl. Phys. B 452 (1995) 109, hep-ph/9503484.

[92] A.D. Martin, W.J. Stirling, R.S. Thorne, Phys. Lett. B 636 (2006) 259, hep-ph/0603143.

[93] M. Spira, et al., Nucl. Phys. B 453 (1995) 17, hep-ph/9504378.

[94] D. Bourilkov, R.C. Group, M.R. Whalley, hep-ph/0605240, 2006. 